\title{
Epitaxial Piezoelectric MEMS on Silicon
}

\author{
THÈSE NO 4939 (2011) \\ PRÉSENTÉE LE 26 JANVIER 2011 \\ À LA FACULTÉ SCIENCES ET TECHNIQUES DE L'INGÉNIEUR \\ LABORATOIRE DE CAPTEURS, ACTUATEURS ET MICROSYSTĖMES \\ SECTION DE MICROTECHNIQUE \\ ÉCOLE POLYTECHNIQUE FÉDÉRALE DE LAUSANNE \\ POUR L'OBTENTION DU GRADE DE DOCTEUR ÈS SCIENCES
}

PAR

Don ISARAKORN

acceptée sur proposition du jury:

Prof. P.-A. Farine, président du jury

Prof. N. de Rooij, Dr D. Briand, directeurs de thèse

Prof. H. Keppner, rapporteur

Prof. H. Shea, rapporteur

Prof. J.-M. Triscone, rapporteur

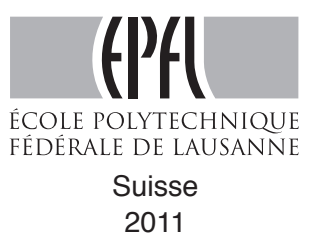





\section{Abstract}

Integration of new functional materials into silicon microsystems is a key factor to enable technology for a wide range of innovative MEMS devices. Piezoelectric materials are of primary interest for integrating sensing and actuation functions in MEMS due to their high forces and high energy densities. The use of PZT thin films in MEMS applications offers the possibility of increasing the sensitivity or actuation capabilities of the devices compared to alternatives such as AlN and $\mathrm{ZnO}$. In general, PZT thin films exhibit smaller piezoelectric coefficients and polarizations than PZT bulk materials due to grain size, composition, crystallographic orientation, non-defined stoichiometry and mechanical boundary conditions. Moreover, PZT thin films are typically grown onto amorphous surfaces resulting in polycrystalline structures, which often lead to degraded performance due to fatigue and aging characteristics. Since epitaxial PZT films exhibit properties, including piezoelectric coefficients, polarizations, and dielectric constants, generally superior to polycrystalline films, it is of high interest to consider their application in MEMS devices, however, there are some real challenges to be solved. Once these issues are overcome, epitaxial PZT thin films could offer interesting potentials in the realization of high performance piezoelectric MEMS.

In this thesis, several aspects related to the development of epitaxial piezoelectric MEMS on silicon are investigated, which cover the following topics: the deposition and integration of high quality epitaxial PZT thin films on silicon wafers; the establishment of microfabrication techniques with associated process flows; and the FEM supported design, and characterization of epitaxial piezoelectric MEMS. A short overview is first given on the current state-of-the-art of piezoelectric MEMS. The integration of epitaxial oxide films on silicon wafers and their properties is then briefly described. The epitaxial oxide thin film heterostructures are based on a piezoelectric $\mathrm{Pb}\left(\mathrm{Zr}_{0.2} \operatorname{Ti}_{0.8}\right) \mathrm{O}_{3}$ layer grown on 2" silicon wafers through two oxide layers: $\mathrm{SrTiO}_{3}$ used as buffer and metallic $\mathrm{SrRuO}_{3}$ used as bottom electrode. The optimized microfabrication process for these oxide layers with specific attention in maintaining the piezoelectric properties of the epitaxial PZT films is presented. The polarization was measured to optimize their processing with at the end no degradation of the piezoelectric properties throughout the process. The epitaxial PZT thin films exhibit a large piezoelectric coefficient $d_{31}$ of 130-140 $\mathrm{pm} \mathrm{V}^{-1}$, allowing the realization of MEMS devices with enhanced actuation/detection properties, such as large amplitude actuation with lower driving voltage, high sensitivity, and high efficiency in energy conversion.

The superior properties of the epitaxial PZT thin film and the effectiveness of the optimized microfabrication techniques have been demonstrated by two examples of epitaxial PZT MEMS devices. First, different epitaxial PZT cantilevers with and without a Si proof mass have been developed for vibration energy harvesting applications. A high power density of up to $14 \mu \mathrm{W} \mathrm{g}{ }^{-2}$ was 
obtained with high current generation and usable voltage, while maintaining lower optimal resistive load. The second application is based on an epitaxial PZT membrane to produce a resonating device. The study of basic characteristics of such device has shown excellent results as it shows a strong harmonic oscillation response with a high quality factor at atmospheric pressure. The finite element model of the epitaxial PZT membrane has then been developed for localized-mass sensing application to determine the resonant frequency, and the effect of the position of the mass and of the resonant mode on the mass sensitivity. The mass sensitivity is of the order $10^{-12} \mathrm{~g} \mathrm{~Hz}^{-1}$, which is in excellent agreement with the simulated value. The minimum detectable mass of $\sim 5 \mathrm{ng}$ can be achieved. These results indicate that the integration of epitaxial thin films on silicon is a promising technology, which improves the performances of piezoelectric MEMS devices. Finally, the concept of charge integration technique for static measurement in piezoelectric sensors is proposed. This technique improves the detection sensitivity of piezoelectric sensors in low frequency measurements, which makes them suitable for chemical and biological detection in a liquid environment.

Keywords: epitaxial oxide, piezoelectric thin film, lead zirconate titanate, PZT, PVDF, energy harvester, mass sensor, membrane, cantilever, charge integration technique, static measurement. 


\section{Résumé}

L'intégration de nouveaux matériaux fonctionnels dans des microsystèmes en silicium est un point crucial pour le développement technologique innovateur de MEMS. Les matériaux piézoélectriques sont de première importance pour l'implémentation de détecteurs et d'actionneurs dus à leur grande densité de force et d'énergie. L'utilisation de couche mince de PZT dans des applications MEMS offre la possibilité d'augmenter la sensibilité d'actuation par rapport à des matériaux alternatifs comme de l'AlN et du ZnO. En général, les couches minces de PZT présentent de plus petits coefficients piézoélectriques et de plus petites valeurs de polarisation que le matériau massif dus à la taille des grains, leur composition, leur orientation cristallographique, leur stæchiométrie non définie et leurs précontraintes mécaniques. De plus, les couches minces de PZT croissent typiquement sur des surfaces amorphes conduisant à des structures polycristallines qui procurent souvent de plus faibles performances à cause de la fatigue et du vieillissement. Puisque des films de PZT épitaxiaux montrent des propriétés généralement supérieures aux films polycristallins, comme leurs coefficients piézoélectriques, leurs polarisations et leurs constantes diélectriques, il est d'un grand intérêt de considérer leur application dans des MEMS. Cependant, de réels challenges subsistent. Une fois que ceux-ci seront résolus, les couches minces de PZT épitaxiées offriraient un potentiel intéressant pour la réalisation de MEMS piézoélectriques de hautes performances.

Dans cette thèse, plusieurs aspects liés au développement de films piézoélectriques épitaxiaux sur silicium couvrant les domaines suivants sont investigués : la déposition et l'intégration de couches minces épitaxiales de PZT de haute qualité sur des substrats en silicium, l'établissement de techniques de microfabrication avec leurs procédés associés, leur conception appuyée par des simulations par éléments finis et la caractérisation de MEMS piézoélectriques. Dans un premier temps, nous donnons un état de l'art actuel sur les MEMS piézoélectriques. Ensuite, l'intégration et les propriétés de films d'oxydes épitaxiaux sur silicium sont brièvement décrites. Dans ce travail, les hétérostructures de couches minces d'oxydes épitaxiaux sont basées sur une couche piézoélectrique de $\mathrm{Pb}\left(\mathrm{Zr}_{0.2} \operatorname{Ti}_{0.8}\right) \mathrm{O}_{3}$ crue sur deux couches d'oxyde sur un substrat en silicium de $50 \mathrm{~mm}$ de diamètre : du $\mathrm{SrTiO}_{3}$ comme couche tampon et du $\mathrm{SrRuO}_{3}$ comme électrode conductrice inférieure. Une attention spécifique a été portée à l'optimisation des procédés de fabrication de ces oxydes afin de maintenir les propriétés piézoélectriques des films épitaxiaux de PZT. La polarisation a été mesurée lors de leur fabrication avec à la fin aucune dégradation des films observée. Les couches minces épitaxiales de PZT présentent un grand coefficient piézoélectrique $d_{31}$ de $130-140 \mathrm{pm} \mathrm{V}^{-1}$ permettant la réalisation de dispositifs avec des propriétés d'actuation/détection améliorées, telles qu'une grande amplitude d'actuation pour une faible tension d'alimentation, une grande sensibilité et un haut rendement en conversion d'énergie. 
Les propriétés supérieures des couches minces épitaxiales de PZT et l'application du procédé de microfabrication ont été démontrées à travers la réalisation de deux exemples de dispositifs. Premièrement, différents leviers en PZT épitaxial avec et sans masse de silicium à leur extrémité ont été développés pour des applications de récupération d'énergie à partir de vibrations. Une densité de puissance aussi importante que $14 \mu \mathrm{W} g^{-2}$ fut obtenue avec la génération d'un courant élevé et une tension utile tout en maintenant une résistance de charge optimale basse. La deuxième application est basée sur une membrane en PZT épitaxial pour produire un système résonant. L'étude des caractéristiques de base d'un tel dispositif a montré d'excellents résultats avec une forte réponse harmonique liée à un facteur de qualité élevé à pression ambiante. Le modèle par éléments finis de la membrane en silicium couverte d'une couche mince de PZT épitaxié a ensuite été élargi à la mesure localisée de masse afin de déterminer la fréquence de résonance, et l'effet de la position de la masse et du mode de résonance. L'ordre de grandeur de la sensibilité en masse obtenue est de $10^{-12} \mathrm{~g} \mathrm{~Hz}^{-1}$, et est en accord avec les résultats des simulations. Nous avons atteint une masse détectable minimum de l'ordre de $5 \mathrm{ng}$. Ces résultats démontrent que l'intégration de couches minces épitaxiales sur silicium est une technologie prometteuse qui améliore les performances des MEMS piézoélectriques.

Finalement, nous proposons le concept d'intégration de charges pour des mesures statiques. Cette technique améliore la sensibilité des capteurs piézoélectriques opérant à basses fréquences, ce qui les rend compatibles avec des mesures chimiques ou biologiques dans des milieux liquides.

Mot-clés : Oxyde épitaxial, couche mince piézoélectrique, oxyde de plomb zirconium et titane, PZT, PVDF, récupérateur d'énergie, capteur massique, membrane, levier, technique d'intégration de charges, mesure statique. 


\section{Contents}

$\begin{array}{ll}\text { Abstract } & \text { i }\end{array}$

Résumé

$\begin{array}{lcl}\text { Contents } & \text { v }\end{array}$

List of journal articles $\quad$ ix

$\begin{array}{lll}\text { Chapter } 1 \quad \text { Introduction } & 1\end{array}$

1.1 Piezoelectricity 1

1.2 Governing equations of piezoelectricity 2

1.3 Motivations 3

$1.4 \quad$ Research context and objectives 4

1.5 Thesis structure 6

$\begin{array}{ll}\text { References } & 8\end{array}$

Chapter 2 State-of-the-art of piezoelectric MEMS 11

$2.1 \quad$ Aluminum nitride and zinc oxide $\quad 11$

2.2 Ferroelectric thin films 12

2.3 Comparison of piezoelectric properties 13

$2.4 \quad$ Piezoelectric MEMS structures and operation modes 14

2.5 Piezoelectric MEMS based on epitaxial PZT thin films 15

$\begin{array}{ll}\text { References } & 17\end{array}$

$\begin{array}{lll}\text { Chapter } 3 & \text { Epitaxial piezoelectric MEMS on silicon (Paper I) }\end{array}$

$\begin{array}{ll}\text { Abstract } & 21\end{array}$

$\begin{array}{lll}3.1 & \text { Introduction } & 22\end{array}$

3.2 Epitaxial thin films growth on silicon 23

3.3 Microfabrication 25

3.3.1 Top electrode materials 25

$\begin{array}{lll}3.3 .2 & \text { Process flow } & 26\end{array}$

$3.4 \quad$ Results and discussion $\quad 28$

3.4.1 Static behavior 28

3.4.2 Dynamic behavior 32

3.5 Conclusions 36

Acknowledgement $\quad 37$

$\begin{array}{ll}\text { References } & 38\end{array}$ 
Chapter 4 Electrical characteristics of piezoelectric energy harvesters based on an epitaxial $\mathrm{Pb}\left(\mathrm{Zr}_{0.2} \mathrm{Ti}_{0.8}\right) \mathrm{O}_{3}$ thin film (Paper II) 41

Abstract 41

$\begin{array}{lll}4.1 & \text { Introduction } & 42\end{array}$

4.2 Device fabrication and characteristics 42

4.3 Results and discussion $\quad 45$

$\begin{array}{lll}4.4 & \text { Conclusions } & 47\end{array}$

$\begin{array}{ll}\text { Acknowledgement } & 47\end{array}$

$\begin{array}{ll}\text { References } & 48\end{array}$

Chapter 5 The realization and performance of vibration energy harvesting MEMS devices based on an epitaxial piezoelectric thin film (Paper III) 51

Abstract

5.1 Introduction $\quad 52$

5.2 Principle and design 54

5.2.1 Analytical power model for piezoelectric energy harvesters 54

5.2.2 Resonant frequency 55

5.2.3 Selection of $\mathrm{Zr} / \mathrm{Ti}$ composition for PZT energy harvesters 56

$\begin{array}{lll}5.3 & \text { Fabrication } & 58\end{array}$

5.3.1 Epitaxial PZT thin films growth on silicon 58

$\begin{array}{lll}5.3 .2 & \text { Device fabrication } & 59\end{array}$

$\begin{array}{lll}5.4 & \text { Results and discussion } & 60\end{array}$

5.4.1 Resonance characterization $\quad 61$

5.4.2 Electromechanical coupling coefficient 62

5.4.3 Mechanical damping ratio 63

$\begin{array}{lll}\text { 5.4.4 Energy harvesting performance } & 64\end{array}$

5.4.5 A comparison of piezoelectric energy harvesting devices 68

$\begin{array}{lll}5.5 & \text { Conclusions } & 70\end{array}$

$\begin{array}{ll}\text { Acknowledgement } & 70\end{array}$

$\begin{array}{ll}\text { References } & 71\end{array}$

Chapter 6 Finite element analysis and experiments on a silicon membrane actuated by an epitaxial PZT thin film for localized-mass sensing $\begin{array}{ll}\text { applications (Paper IV) } & 77\end{array}$

$\begin{array}{ll}\text { Abstract } & 77\end{array}$

$\begin{array}{lll}6.1 & \text { Introduction } & 79\end{array}$

6.2 Mass sensing performance - theoretical background 80 
6.2.1 Mass sensitivity 81

6.2.2 Limit of detection 81

$\begin{array}{lll}\text { 6.2.3 Quality factor } & 82\end{array}$

6.3 Growth of epitaxial thin films on silicon 82

$\begin{array}{lll}6.4 & \text { Device fabrication } & 83\end{array}$

6.5 Finite element analysis $\quad 84$

6.5.1 Resonant frequency simulation 85

6.5.2 Mass-change sensitivity 86

6.6 Experimental results and discussion $\quad 88$

6.6.1 Resonance characterization 89

6.6.2 Mass loading and sensing 91

$\begin{array}{lll}6.7 & \text { Conclusions } & 95\end{array}$

Acknowledgement 95

References 96

Chapter 7 Evaluation of static measurement in piezoelectric cantilever sensors using a charge integration technique for chemical and biological detection (Paper V) 101

$\begin{array}{ll}\text { Abstract } & 101\end{array}$

$\begin{array}{lll}7.1 & \text { Introduction } & 102\end{array}$

$\begin{array}{lll}7.2 & \text { Charge integration technique } & 102\end{array}$

$\begin{array}{ll}\text { 7.2.1 Piezoelectric cantilevers } & 103\end{array}$

$\begin{array}{ll}\text { 7.2.2 Precision switched integrator } & 104\end{array}$

$\begin{array}{lll}7.2 .3 & \text { Reset-and-integrate operation } & 105\end{array}$

$\begin{array}{lll}7.3 & \text { Results and discussion } & 106\end{array}$

$\begin{array}{lll}\text { 7.3.1 Control experiments } & 106\end{array}$

$\begin{array}{ll}\text { 7.3.2 Alkanethiol detection } & 110\end{array}$

$\begin{array}{lll}7.4 & \text { Conclusions } & 113\end{array}$

$\begin{array}{ll}\text { References } & 115\end{array}$

$\begin{array}{llr}\text { Chapter } 8 & \text { Conclusions and outlook } & 119\end{array}$

$\begin{array}{lr}\text { Acknowledgements } & 123\end{array}$

$\begin{array}{lr}\text { Publications } & 125\end{array}$

$\begin{array}{lr}\text { Biography } & 127\end{array}$ 



\section{List of journal articles}

The structure of this thesis is organized based on the following journal articles:

Paper I Epitaxial piezoelectric MEMS on silicon

D. Isarakorn, A. Sambri, P. Janphuang, D. Briand, S. Gariglio, J. -M. Triscone,

F. Guy, J. W. Reiner, C. H. Ahn, and N. F. de Rooij

Journal of Micromechanics and Microengineering, Vol. 20, 055008, 2010.

Paper II Electrical characteristics of piezoelectric energy harvesters based on an epitaxial $\operatorname{Pb}\left(\mathrm{Zr}_{0.2} \mathrm{Ti}_{0.8}\right) \mathrm{O}_{3}$ thin film

D. Isarakorn, D. Briand, P. Janphuang, N. F. de Rooij, J. W. Reiner, and C. H. Ahn (submitted).

Paper III The realization and performance of vibration energy harvesting MEMS devices based on an epitaxial piezoelectric thin film

D. Isarakorn, D. Briand, P. Janphuang, A. Sambri, S. Gariglio, J. -M. Triscone,

F. Guy, J. W. Reiner, C. H. Ahn, and N. F. de Rooij

Smart Materials and Structures (accepted).

Paper IV Finite element analysis and experiments on a silicon membrane actuated by an epitaxial PZT thin film for localized-mass sensing applications

D. Isarakorn, D. Briand, A. Sambri, S. Gariglio, J. -M. Triscone, F. Guy,

J. W. Reiner, C. H. Ahn, and N. F. de Rooij

Sensors and Actuators, B: Chemical, 2010, doi: 10.1016/j.snb.2010.10.009.

Paper V Evaluation of static measurement in piezoelectric cantilever sensors using a charge integration technique for chemical and biological detection

D. Isarakorn, M. Linder, D. Briand, and N. F. de Rooij

Measurement Science and Technology, Vol. 21, 075801, 2010. 



\section{Chapter 1}

\section{Introduction}

This chapter provides an introduction to the research that was conducted throughout the course of this study. First, a brief explanation of the piezoelectric theory is given. Next, the motivation of this research as well as the research context and objectives are presented. Finally, a summary of the thesis structure is provided.

\subsection{Piezoelectricity}

Piezoelectricity is a coupling between the mechanical and electrical behaviors of a material. The piezoelectric effect in quartz was discovered in 1880 by the brothers J. Curie and P. Curie. When certain types of crystals are subjected to tensile or compressive forces, the resulting strain causes a polarized state in the crystal, and an electric field is created. This phenomenon is called direct piezoelectric effect. Conversely, if a crystal is polarized by an electric field, strains along with resulting stresses are created, which was called converse piezoelectric effect. In crystals that show piezoelectric properties, electrical quantities such as electric field or polarization, and mechanical quantities such as stress or strain, are interrelated. This phenomenon is called electromechanical coupling. In piezoelectric materials, the transverse ( $d_{31}$ mode) and longitudinal ( $d_{33}$ mode) effects are very important. In $d_{33}$ mode, the direction of applied stress (force) and generated charge is the same, while in $d_{31}$ mode the stress is applied in one axial direction but the charge is obtained from the perpendicular direction as shown in figure 1.1.

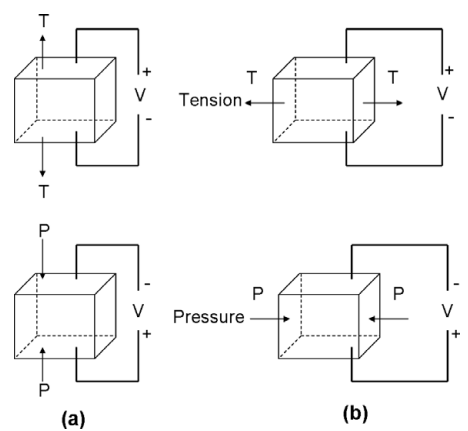

Figure 1.1 The piezoelectric effect: when a pressure (P) or a tension $(\mathrm{T})$ is applied to a crystal, a voltage (V) develops across the material. (a) When the deformation and voltage are collinear, this is the $d_{33}$ mode (longitudinal effect). (b) In the case of perpendicular deformation and voltage, the mode is $d_{31}$ (transverse mode). 
In general, a piezoelectric material has a polycrystalline structure consisting of many domains. Each domain has a polarization, one end is more negatively charged and the other end is positively charged. Let us assume the polarization is an imaginary line that runs through the center of both charges on the domain as shown in figure 1.2. In order to obtain a net polarization in piezoelectric materials, the polarization of all domains must lie in one direction. The process so-called poling must be performed on a multidomain piezoelectric material in order to produce the piezoelectric effect. Poling is the process by which electric field is applied to a piezoelectric material (figure 1.2). At a certain voltage, the polarization in all domains lines up and face in nearly the same direction. The piezoelectric effect can now be observed in the piezoelectric material.

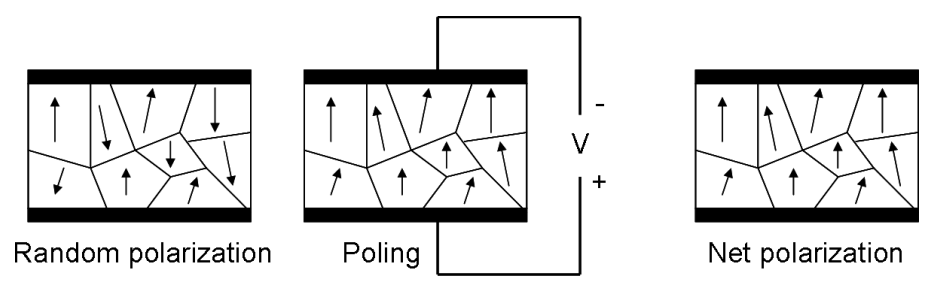

Figure 1.2 Poling process to generate piezoelectric effect.

The piezoelectric effect provides the ability to use piezoelectric materials as both a sensor and an actuator. Piezoelectric materials are therefore widely used in many areas of technology and science. It should be noted that the poling process is not necessary for the epitaxial piezoelectric films since they are naturally polarized.

\subsection{Governing equations of piezoelectricity}

Piezoelectricity that combines electrical and mechanical components described by Gauss's Law and Hooke's Law can be expressed by the following relations:

$$
\begin{aligned}
& D=\varepsilon_{0} E+P \\
& S=s T
\end{aligned}
$$

where $D$ is the electric charge density displacement, or the electric displacement $\left[\mathrm{C} \mathrm{m}^{-2}\right], \varepsilon$ is the permittivity $\left[\mathrm{F} \mathrm{m}^{-1}\right], E$ is the electric field strength $\left[\mathrm{V} \mathrm{m}^{-1}\right], P$ is the polarization, $S$ is the mechanical strain, $s$ is the compliance $\left[\mathrm{m} \mathrm{N}^{-1}\right]$, and $T$ is the applied mechanical stress $\left[\mathrm{N} \mathrm{m}^{-2}\right]$. A relation between stress, strain, and electric field can be demonstrated through equation 1.3: 


$$
S_{i}=d_{k i} E_{k}+s_{i j}^{E} T_{j}
$$

where $S_{i}$ is the part of the strain produced by the electric field $\left(E_{k}\right)$, the piezoelectric constant $\left(d_{k i}\right)$ is the part of the strain produced by the applied stress $\left(T_{j}\right)$, and $s_{i j}^{E}$ is the elastic compliance coefficient under the condition of a constant electric field. Meanwhile, the electric displacement, electric field, and stress can be related by equation 1.4 :

$$
D_{l}=\varepsilon_{l n}^{T} E_{n}+d_{l m} T_{m}
$$

where $\varepsilon_{l n}^{T}$ is the dielectric strain coefficient, or permittivity, under the constant stress $\left(T_{m}\right), D_{l}$ is the dielectric displacement, and the subscript values for the terms in equations 1.3 and 1.4 are $\mathrm{i}, \mathrm{j}, \mathrm{m}=$ $1, \ldots, 6$ and $\mathrm{k}, 1, \mathrm{n}=1,2,3$. Equations 1.3 and 1.4 comprise the relationship between the electrical and mechanical properties of piezoelectric materials.

\subsection{Motivation}

Microelectromechanical systems (MEMS) are making an impact in a wide range of applications, allowing creation of new types of sensors, actuators, and transducer systems. Early MEMS devices were based solely on silicon by leveraging many of the materials and processes transferred from a well-developed IC industry. As the MEMS technology has grown over the years, there is always further need for more complex functions of MEMS devices. This requirement can be achieved by adding a variety of functional materials onto silicon with optimal fabrication processes. An important family of functional materials is piezoelectric materials, which can provide a direct transduction mechanism to convert signals from mechanical to electrical domains and vice versa. Moreover, piezoelectric materials are high energy density materials that are suitable for miniaturization. Therefore, this has led to a growing interest in piezoelectric thin films for MEMS applications.

The most used piezoelectric thin film materials in MEMS devices are zinc oxide ( $\mathrm{ZnO})$, lead zirconate titanate (PZT), and aluminum nitride (AIN). MEMS devices using $\mathrm{ZnO}$ and AlN films have been successfully demonstrated $[1,2]$. Both $\mathrm{ZnO}$ and AlN films are compatible with existing semiconductor fabrication processes [3]. However, these films exhibit low piezoelectric coefficients that restrict their use to some specific applications, i.e. bulk acoustic wave or sensing applications [4]. In comparison, PZT films have superior ferroelectric and piezoelectric properties, which are very attractive for piezoelectric MEMS sensor and actuator devices.

In MEMS technology, the most important substrate is silicon. PZT thin films, however, cannot be grown directly on it. Buffer layers between PZT and the silicon substrate are specifically needed to prevent interdiffusion and oxidation reactions. For most applications, PZT thin films are 
typically deposited on Pt-coated silicon substrates, which exhibit low polarizations and piezoelectric coefficients due to their polycrystalline structures [5]. In order to improve the piezoelectric properties of the PZT thin films, the interface between the PZT thin films and the silicon substrates must be controlled, and the PZT thin films also need to be oriented to the desired direction [6]. Crystalline oxide materials like $\mathrm{SrTiO}_{3}$ (STO), yttria-stabilized zirconia (YSZ), and $\mathrm{MgO}$, are often used as the interface layer in order to grow highly oriented (epitaxial) PZT thin films on the silicon substrates [710]. The resulting optimally oriented epitaxial layer improves piezoelectric properties in comparison with the polycrystalline PZT thin films on the Pt-coated silicon substrates. Moreover, the fatigue effect and cyclic depolarization are expected to be reduced [11], which is necessary for reliable use in devices. This approach could open the development of the next generation of piezoelectric MEMS devices.

Over the past two decades, a great amount of progress has been successfully achieved in the epitaxial growth of thin films of various oxides, including ferroelectrics [12]. Early substrates used to grow the epitaxial films were based primarily on various single-crystalline oxide substrates for instance $\mathrm{SrTiO}_{3}$ [13], $\mathrm{BaTiO}_{3}$ [14], $\mathrm{LaAlO}_{3}$ [15], and $\mathrm{MgO}$ [16]. Later, such progress has driven research efforts to focus on the integration of these single-crystalline oxide materials directly on silicon substrates. The availability of high-quality oxide films on silicon substrates provides a very attractive opportunity to integrate other materials with a high crystalline quality on such a surface for different applications [17]. The epitaxial ferroelectric PZT is an important material that has been investigated and successfully integrated onto silicon for potential applications in infrared sensors, nonvolatile memory devices, surface acoustic wave (SAW) devices, and especially MEMS [17-19]. However, the epitaxial growth of PZT thin films was limited only on chip-scale substrates. For the fabrication of MEMS, it is necessary to control the growth of these layers on wafer-scale silicon substrates and to develop appropriate fabrication processes to realize micromachined structures [2024]. Nevertheless, the epitaxial piezoelectric MEMS devices are presently only implemented at the chip-scale level mainly due to the difficulty of the epitaxial growth of piezoelectric films on larger areas [25]. The development of appropriate microfabrication processes to realize micromachined structures based on the epitaxial PZT thin films is also considered to be a crucial technological step towards the development of novel MEMS devices. The successful combination of epitaxial PZT thin films on silicon substrates with optimized microfabrication techniques will allow the realization of MEMS devices with enhanced actuation/detection properties. Hence, this thesis presents the establishment of a technology platform for fabrication of epitaxial piezoelectric MEMS devices with the emphasis on resonating devices and energy harvesting applications.

\subsection{Research context and objectives}

The work of this thesis is part of the Materials with Novel Electronic Properties (MaNEP) research program under the NCCR program of the Swiss National Science Foundation that was launched in 
2001 to promote long-term research projects for exploring new electronic materials and their applications. One of sub-projects in MaNEP focused on the realization of high performance MEMS devices based on an epitaxial piezoelectric thin film grown directly on silicon. In collaboration with the group of Prof. Jean-Marc Triscone (University of Geneva), the goal of this MaNEP sub-project was to develop the controlled growth of epitaxial piezoelectric thin films on full silicon wafers through oxide layers, and develop appropriate fabrication process to pattern them to realize micromachined structures for specific applications. The work performed at the group of Prof. Triscone was to develop the epitaxial growth of PZT thin films and related oxides on wafer-scale silicon substrates (2" silicon wafers). Research activities at EPFL-SAMLAB involved the development of microfabrication processes for the epitaxial thin films on silicon, and the realization of epitaxial PZT MEMS devices, which are the main goals of this thesis. In order to achieve these goals, the specific objectives addressed in this thesis are as follows:

- Study the properties of epitaxial oxide films and the techniques for their integration on silicon wafers for the realization of piezoelectric MEMS. The knowledge gained supported the development of compatible microfabrication techniques and the design of the piezoelectric MEMS devices;

- Investigate the degradation in ferroelectric and piezoelectric properties of epitaxial PZT thin films due to microfabrication processes and material used as a top electrode. The goal here is to acquire all the knowledge that is necessary to produce high quality and reproducible epitaxial piezoelectric MEMS devices with special attention on maintaining the superior properties of epitaxial PZT thin films in operating devices for better performance;

- Investigate various microfabrication processes for piezoelectric MEMS and apply the experience obtained to develop efficient micropatterning methods and process sequences for the microfabrication of the epitaxial piezoelectric MEMS devices;

- Verify the applicability of the optimized microfabrication techniques and the usefulness of the piezoelectric properties of the epitaxial PZT thin films. Experimentally, epitaxial PZT cantilevers and membranes were fabricated, and their static and dynamic behaviors were examined. At this stage, the properties of the epitaxial films and the benefits that the epitaxial films brought to piezoelectric MEMS devices were evaluated;

- Develop finite element models for the design of membrane and cantilever based resonating devices. The properties of the epitaxial PZT thin films obtained from measurements were used as inputs for the design and modeling of the devices. The appropriateness of the models was validated by characterization of the fabricated devices; 
- Demonstrate specific applicative devices: a vibration energy harvester and a membrane-based sensor have been selected. The study of concepts, physics, and design and analysis based on finite element and/or analytical models were carried out.

In addition, the aspects related to a read-out circuitry for surface stress detection of piezoelectric sensors operating in the static mode that fall outside the main scope of the current project were also investigated in this thesis.

\subsection{Thesis structure}

This thesis is organized in a collection of the published papers and manuscripts from the author. Apart this first chapter dedicated to the motivations and objectives of this work, the thesis is structured as follow:

Chapter 2 gives an overview of piezoelectric MEMS with specific attention to the piezoelectric materials used. The major properties of piezoelectric thin films are also presented.

Chapter 3 introduces the work performed for the establishment of an epitaxial piezoelectric MEMS technology platform. The details of the epitaxial growth of all oxide layers and the microfabrication processes used for realizing epitaxial piezoelectric MEMS devices on epitaxial $\mathrm{Pb}\left(\mathrm{Zr}_{0.2} \mathrm{Ti}_{0.8}\right) \mathrm{O}_{3}$ thin films are presented. Different issues related to the choice of materials and especially processes used to fabricate the devices and the influence of these on the properties of the epitaxial piezoelectric films are addressed. A focus is dedicated to the microfabrication techniques required to pattern these materials and especially on the influence of the processing temperature and of the type of materials used as top electrodes on the epitaxial piezoelectric films properties. It is found that the thermal effect is a primary cause of the degradation of the properties of piezoelectric thin films when covered with the top electrode material. Membranes and cantilevers are realized using a sequence of microfabrication processes optimized for epitaxial oxide layers. Their static and dynamic behaviors are evaluated. An interesting aspect of the epitaxial PZT transducers is that the operational voltage is low compared to transducers actuated with polycrystalline PZT films. A large displacement can be obtained from the epitaxial PZT films due to excellent piezoelectric properties. Moreover, a strong harmonic oscillation with a high quality factor in dynamic mode can be achieved.

Chapter 4 focuses on vibration energy harvesting applications by utilizing the epitaxial PZT cantilever structures. The microfabrication processes presented in the previous chapter are used to realize the vibration energy harvesting MEMS devices. The electrical characteristics of the epitaxial $\mathrm{Pb}\left(\mathrm{Zr}_{0.2} \mathrm{Ti}_{0.8}\right) \mathrm{O}_{3}$ thin film applied to energy harvesting devices are investigated in the viewpoint of material properties (piezoelectric coefficient and dielectric constant). The main purpose of this study is to demonstrate the benefits of epitaxial PZT thin film for vibration energy harvesting applications. Key parameters of the epitaxial PZT thin film for realizing high performance piezoelectric energy 
harvesters are highlighted. The results reveal that epitaxial PZT thin films have a large piezoelectric coefficient and a low dielectric constant, resulting in high power generation capabilities.

In Chapter 5, based on the results obtained in the Chapter 4, the vibration energy harvesting MEMS devices based on the epitaxial PZT thin films are studied in more details. In the first part, the analytical power and finite element models used to design and optimize the devices are presented. The choice of the actual PZT composition for energy harvesting applications is discussed. In the following part, the results on the fabrication and characterization of the epitaxial PZT cantilevers with and without a Si proof mass are presented. Different characteristics of the fabricated epitaxial PZT harvesters are determined: their dynamic behavior, electromechanical coupling coefficient and electrical characteristics. In the last part, the performances of the epitaxial PZT harvesters are compared with other demonstrated piezoelectric energy harvesters. A very interesting outcome is that the epitaxial PZT harvester generates higher power and current with usable voltage, and requires lower optimal resistive load compared to piezoelectric harvesters realized with polycrystalline PZT or AlN films.

Chapter 6 addresses the development of a silicon membrane actuated by an epitaxial PZT thin film for localized-mass sensing applications. The dynamic behavior and mass sensing performance of the proposed structure are experimentally investigated and compared to numerical analyses. The effect of the mass position and of the resonant mode on the mass sensitivity, as well as the minimum detectable mass of the proposed device is evaluated. It is found that the mass sensitivity is a strong function of the mass position and the vibration mode, being highest when placing mass at the antinode and operating the sensor at higher mode of resonance. The observation of strong harmonic oscillation with a high quality factor implies the feasibility of highly sensitive detection of biomolecules using the epitaxial PZT membranes in liquid environments.

In Chapter 7, a focus is dedicated to the development of static measurement for piezoelectric sensors using a charge integration technique with the aim of applying to chemical and biological detection. The principle of the charge integration technique and the detail of apparatus used to realize the detection system are presented. In this study, the feasibility to detect electrically and statically the deflections of piezoelectric cantilevers using a charge integrator in real time is explored. The proposed technique is demonstrated with the detection of alkanethiols using Au-coated Polyvinylidene Fluoride (PVDF) piezoelectric cantilevers in liquid environment. The outcome of the study should incite to the implementation of the static detection method of piezoelectric cantilever sensors for various applications.

Finally, a summary of the results from this work along with some potential future research directions in the development of epitaxial piezoelectric MEMS are presented in Chapter 8. 


\section{References}

[1] Ruby R 2007 Review and comparison of bulk acoustic wave FBAR, SMR technology Proc. IEEE Ultrasonic Symp. vol 7 pp 1029-40

[2] De Voe D L and Pisano A P 2001 Surface micromachined piezoelectric accelerometers (PiXLs) J. Microelectromech. Syst. 10 180-6

[3] Vellekoop M J, Visser C C O, Sarro P M and Venema A 1990 Compatibility of zinc oxide with silicon IC processing Sens. Actuators, A 23 1027-30

[4] Tadigadapa S and Mateti 2009 Piezoelectric MEMS sensors: state-of-the-art and perspectives Meas. Sci. Technol. 20092001

[5] Muralt P 1997 Piezoelectric thin films for MEMS Integr. Ferroelectr. 17 297-307

[6] Ramesh R and Schlom D G 2002 Orienting Ferroelectric Films Science 296 1975-1976

[7] Kanno I, Fujii S, Kamada T and Takayama R 1997 Piezoelectric properties of $c$-axis oriented $\mathrm{Pb}(\mathrm{Zr}, \mathrm{Ti}) \mathrm{O}_{3}$ thin films Appl. Phys. Lett. 70 1378-80

[8] Kanno I, Kotera H and Wasa K 2003 Measurement of transverse piezoelectric properties of PZT thin films Sens. Actuators, A 107 68-74

[9] Lin A, Hong X, Wood V, Verevkin A A, Ahn C H, McKee R A, Walker F J and Specht E D 2001 Epitaxial growth of $\mathrm{Pb}\left(\mathrm{Zr}_{0.2} \mathrm{Ti}_{0.8}\right) \mathrm{O}_{3}$ on $\mathrm{Si}$ and its nanoscale piezoelectric properties Appl. Phys. Lett. 78 2034-36

[10] Dekkers M, Nguyen M D, Steenwelle R, te Riele P M, Blank D H A and Rijnders G 2009 Ferroelectric properties of epitaxial $\mathrm{Pb}(\mathrm{Zr}, \mathrm{Ti}) \mathrm{O}_{3}$ thin films on silicon by control of crystal orientation Appl. Phys. Lett. 95012902

[11] Guerrero C, Ferrater C, Roldan J, Trtik V, Sanchez F and Varela M 2000 Epitaxial ferroelectric $\mathrm{PbZr}_{\mathrm{x}} \mathrm{Ti}_{1-\mathrm{x}} \mathrm{O}_{3}$ thin films for non-volatile memory applications Microelectron. Reliab. 40 671-74

[12] Rabe K, Ahn C H and Triscone J -M 2007 Physics of ferroelectrics a modern perspective (Springer) p 219

[13] Kim D M, Eom C B, Nagarajan V, Ouyang J, Ramesh R, Vaithyanathan V and Schlom D G 2006 Thickness dependence of structural and piezoelectric properties of epitaxial $\mathrm{Pb}\left(\mathrm{Zr}_{0.52} \mathrm{Ti}_{0.48}\right) \mathrm{O}_{3}$ films on $\mathrm{Si}$ and $\mathrm{SrTiO}_{3}$ substrates Appl. Phys. Lett. 88142904

[14] Kay H F and Vousden P 1949 Symmetry changes in barium titanate at low temperatures and their relation to its ferroelectric properties Philos. Mag. 40 1019-40

[15] Wang S, Wang L and Gu B 2008 High quality YBCO film growth on $\mathrm{SrTiO}_{3}$-buffered $\mathrm{LaAlO}_{3}$ substrate by full solution method J. Mater. Sci. Technol. 24 899-902

[16] Kanno I, Fujii S, Kamada T and Takayama R 1997 Piezoelectric properties of $c$-axis oriented $\mathrm{Pb}(\mathrm{Zr}, \mathrm{Ti}) \mathrm{O}_{3}$ thin films Appl. Phys. Lett. 70 1378-80 
[17] Wang Y, Ganpule C, Liu B T, Li H, Mori K, Hill B, Wuttig M, Ramesh R, Finder J, Yu Z, Droopad R and Eisenbeiser K 2002 Epitaxial ferroelectric $\mathrm{Pb}(\mathrm{Zr}, \mathrm{Ti}) \mathrm{O}_{3}$ thin films on $\mathrm{Si}$ using $\mathrm{SrTiO}_{3}$ template layers Appl. Phys. Lett. 80 97-9

[18] Scott J F 2007 Applications of modern ferroelectrics Science 315 954-9

[19] Scott J F and Paz de Araujo C A 1989 Ferroelectric memories Science 246 1400-5

[20] Muralt P 2000 PZT thin films for microsensors and actuators: Where do we stand? IEEE Trans. Ultrason. Ferroelectr. Freq. Control. 47 903-15

[21] Muralt P 2000 Ferroelectric thin films for micro-sensors and actuators: a review $J$. Micromech. Microeng. 10 136-46

[22] Damjanovic D, Muralt P and Setter N 2001 Ferroelectric sensors IEEE Sens. J. 1 191-206

[23] Trolier-McKinstry S and Muralt P 2004 Thin film piezoelectrics for MEMS J. Electroceram. 12 7-17

[24] Baborowski J 2004 Microfabrication of piezoelectric MEMS Integr. Ferroelectr. 66 3-17

[25] Reilly E K and Wright P K 2009 Modeling, fabrication and stress compensation of an epitaxial thin film piezoelectric microscale energy scavenging device J. Micromech. Microeng. 19095014 



\section{Chapter 2}

\section{State-of-the-art of piezoelectric MEMS}

Piezoelectric thin films offer a number of advantages in MEMS applications due to their efficient voltage-deflection conversion, high energy densities, low noise, high frequency operation, and low power requirements. In MEMS technology, the most used piezoelectric thin films are pyroelectric $\mathrm{AlN}$ and $\mathrm{ZnO}$ (in their wurtzite structure) and ferroelectric PZT. The major difference between ferroelectric and pyroelectric is the possibility of reorienting the internal polarization upon application of an electric field: possible in ferroelectrics with fields above the material coercive field, it is absent in pyroelectrics. The most widely used ferroelectric thin films for MEMS applications are based on lead zirconate titanate (PZT). The choice of the piezoelectric thin films for MEMS depends on the targeted applications. In this chapter, the piezoelectric thin film materials, their properties, their integration into MEMS structures, and their modes of operation are reviewed.

\subsection{Aluminum nitride and zinc oxide}

Both aluminum nitride (AlN) and zinc oxide $(\mathrm{ZnO})$ are non-ferroelectric materials exhibiting a piezoelectric response along the [0001] crystallographic direction. Consequently, to achieve good piezoelectric effect, particular growth processes are required. For MEMS applications, AIN and ZnO are commonly sputtering deposited [1]. $\mathrm{ZnO}$ is deposited usually close to room temperature in order to avoid a fast diffusing $\mathrm{Zn}$ ion and to obtain a high resistivity, while high-quality AlN can be obtained at low temperature below $200{ }^{\circ} \mathrm{C}$ [2]. In the early stage of the development of piezoelectric MEMS, $\mathrm{ZnO}$ was the first one to be demonstrated due to the availability of $\mathrm{ZnO}$ and easily processing [3]. $\mathrm{ZnO}$ thin films have been applied in many MEMS devices, including acoustic sensors and accelerometers as well as in bulk acoustic wave (BAW) devices [4]. MEMS devices based on AIN thin films have been demonstrated mainly for sensor applications because AlN thin films have low permittivity and low dielectric loss giving a better signal-to-noise ratio and higher output voltage [5]. One of the most interesting features of piezoelectric $\mathrm{ZnO}$ and AlN thin films lies in the fact that they are fully compatible with the standard CMOS technology [6]. However, these films exhibit small piezoelectric coefficients that restrict their use to acoustic resonators and sensors. For actuator and transducer applications, ferroelectric thin films are more appropriate because their piezoelectric coefficients are much higher than those of $\mathrm{ZnO}$ and $\mathrm{AlN}$ thin films. 


\subsection{Ferroelectric thin films}

Lead zirconate titanate (PZT) is the most widely used ferroelectric thin films for piezoelectric MEMS where large piezoelectric coefficients are required. The large piezoelectric coefficients of PZT films are very attractive for various microactuator and microsensor applications. The fabrication of PZT thin films has been leveraged from the tremendous infrastructure development resulting from ferroelectric random access memories, which definitely accelerate the development of ferroelectricsbased MEMS devices. Currently, high-quality PZT thin films can be grown by several techniques such as sputtering [7-9], pulse laser deposition (PLD) [10-12], sol-gel [13-15], metalorganic chemical vapor deposition (MOCVD) [16-18], and plasma enhanced chemical vapor deposition (PECVD) [19]. Typically, these growth methods involve three main steps: (1) generation of atomic or molecular species of interest, (2) transport and deposition on the substrate, and (3) post-deposition crystallization and annealing of the film [4]. These deposition methods have shown varying advantages in terms of deposition rate and temperature, film uniformity and conformability, compositional control, substrate size, and cost. The details of these techniques and the issues relating to the conditions used for the growth process and quality control of the films can be found in the review articles [1,20-22].

In MEMS devices, PZT thin films are grown on a conductive electrode. The choice of the bottom electrode is of primary importance as it reflects the crystalline texture and the properties of the PZT thin films. The most often used materials for the bottom electrode include metal oxides $\left(\mathrm{RuO}_{2}\right)$, perovskite metal oxides $\left(\mathrm{SrRuO}_{3}\right)$, and platinum. The later is now widely used in MEMS devices. A number of applications based on PZT thin films grown on platinized silicon wafers have been successfully demonstrated with interesting performance [20-22]. However, PZT thin films often exhibit smaller polarizations and piezoelectric coefficients than PZT bulk ceramics due to the polycrystalline nature of the PZT thin films [23]. Therefore, there is still some room for the maximization of the piezoelectric properties to achieve a performance close to the best ceramics used today.

In order to improve the properties of the PZT thin films, research efforts have been focused on the crystallization and orientation of these films and on the optimization of the interface between the film and the electrode. Over the past two decades, a tremendous amount of progress has been achieved in the epitaxial growth of the PZT thin films [24]. One important way is to choose the appropriate substrate to lower the strain introduced by the mismatch of the substrate and the PZT thin films as well as to control the PZT orientation to the desired direction. The continuous improvement in the epitaxial growth techniques provides an opportunity to grow high-quality oxide films as a template layer on silicon substrates. With such a configuration, epitaxial PZT thin films can be grown on silicon with a high crystalline quality and the piezoelectric and ferroelectric properties are much improved in comparison with polycrystalline PZT on platinized silicon wafers [23].

In the past few years, much interest has also been focused on relaxor ferroelectric thin films. Relaxors are lead-containing perovskites, exhibiting anomalous characteristics typified by a strong 
frequency dispersion of the permittivity, while relaxor ferroelectrics are solid solutions of relaxors and normal ferroelectrics, such as $\mathrm{PMN}-\mathrm{PbTiO}_{3}$ and $\mathrm{PZN}-\mathrm{PbTiO}_{3}$. One of the most studied relaxor ferroelectric material is lead magnesium niobate-lead titanate (PMN-PT) [25]. PMN-PT materials exhibit large electrostrictive strain, high permittivity over a wide temperature range, strong piezoelectric coefficients, and extremely large electromechanical coupling coefficients compared to PZT [4, 26], which make them a great interest in the field of piezoelectric MEMS [25]. However, PMN-PT thin films suffer from low transition temperatures leading to early depoling, limitations on the available drive levels due to low coercive fields, and instability of the piezoelectric response at increasing temperatures [1]. Research in relaxor ferroelectric thin films and their integration on silicon is still ongoing. Once these issues have been solved, relaxor ferroelectric thin films offer interesting potentials in the microfabrication of high performance piezoelectric MEMS.

\subsection{Comparison of piezoelectric properties}

Typical piezoelectric properties of different piezoelectric films are summarized in table 1. The effective piezoelectric coefficients $e_{31, f}$ and $d_{33, f}$ are introduced to describe the piezoelectric coefficients of the clamping piezoelectric thin films experienced in a composite structure, usually with silicon substrates. These effective piezoelectric coefficients are not the intrinsic property of the piezoelectric materials, but they take into account the properties of the substrates. The effective piezoelectric coefficients can be calculated as [27]:

$$
\begin{aligned}
& e_{31, f=}=\frac{d_{31}}{s_{11}^{E}+s_{12}^{E}} \\
& d_{33, f}=d_{33}-\frac{2 s_{13}^{E}}{s_{11}^{E}+s_{12}^{E}} d_{31}
\end{aligned}
$$

where $s_{i j}^{E}$ is the elastic compliance when the electric field is held constant. The effective piezoelectric coefficients of piezoelectric thin films are thus lower than the bulk values due to the clamping effect.

Referring to table 2.1, AlN and $\mathrm{ZnO}$ thin films exhibit quite similar piezoelectric properties. The transverse piezoelectric coefficients $e_{31, f}$ of PZT and PMN-PT thin films are more than 10 times higher than those observed in ZnO or AlN. Therefore, PZT and PMN-PT thin films are suitable for actuators that require high force, motion, and output power and for sensors for which high current is needed. However, the piezoelectric coefficients are not the only material properties of interest, other properties such as dielectric constants and dielectric losses are also important, which reflect to the performance in different applications. For instance, a low value of dielectric loss angle $(\tan \delta)$ can be 
found in AIN, and thus it is a perfect material for sensor applications where an excellent signal-tonoise ratio is required. Moreover, AlN thin films have low permittivity, allowing higher voltage generation. This property is very attractive for vibration energy harvesting applications [28].

Table 2.1. Properties of piezoelectric thin films.

\begin{tabular}{lcccc}
\hline Parameter & ZnO [1] & AlN [1] & PZT [1] & PMN-PT [29] \\
\hline$e_{31, f}\left(\mathrm{Cm}^{-2}\right)$ & -1.0 & -1.05 & -8 to -12 & -9 to -20 \\
$d_{33, f}\left(\mathrm{pmV}^{-1}\right)$ & 5.9 & $3.9-5.5$ & $60-130$ & $100-350$ \\
$\varepsilon_{33}$ & 10.9 & 10.5 & $300-1300$ & $1000-3000$ \\
$\tan \delta\left(10^{5} \mathrm{Vm}^{-1}\right)$ & $0.01-0.1$ & 0.003 & $0.01-0.03$ & \\
\hline
\end{tabular}

\subsection{Piezoelectric MEMS structures and operation modes}

Most piezoelectric MEMS sensor and actuator devices are based on laminated piezoelectric/substrate planar structures. Various geometry and clamping conditions can be considered depending on applications and the most common examples are as follows:

- Cantilevers: atomic force microscopy (AFM), biosensors, chemical sensors, and energy harvesting devices;

- Bridges: resonators, mass sensors, RF switches, and acoustic sensors;

- Membranes: pressure sensors, micromotors, accelerometers, and micropumps.

In general, mechanical bending of the structure can greatly amplify the displacement generated by the piezoelectric films for actuator applications or the applied stress on the piezoelectric films for sensor applications. At present, many designs for piezoelectric MEMS devices utilized the polycrystalline PZT thin films, which allow the operations in either $d_{31}$ or $d_{33}$ modes. The $d_{31}$ mode transducers have a PZT layer in between top and bottom electrodes, while the $d_{33}$ mode transducers eliminate the need for a bottom electrode by utilizing interdigit (IDT) electrodes on the top of a PZT layer as illustrated in figure 2.1. In $d_{31}$ mode, the polarization direction is through the film's thickness and perpendicular to the plane of the film, but the generated displacement to be used or the applied stress to be detected is in the plane of the film. On the other hand, the use of PZT films that are polarized in the plane of the film instead of through the thickness enables the $d_{33}$ mode to be employed. The $d_{33}$ piezoelectric coefficient is typically around twice the magnitude of the $d_{31}$ piezoelectric coefficient in PZT [30]. Therefore, the use of $d_{33}$ mode could enhance the sensing/actuation properties of the devices. However, depending on the applications, other properties such as the dielectric constant have to be considered when selecting the operation mode. 

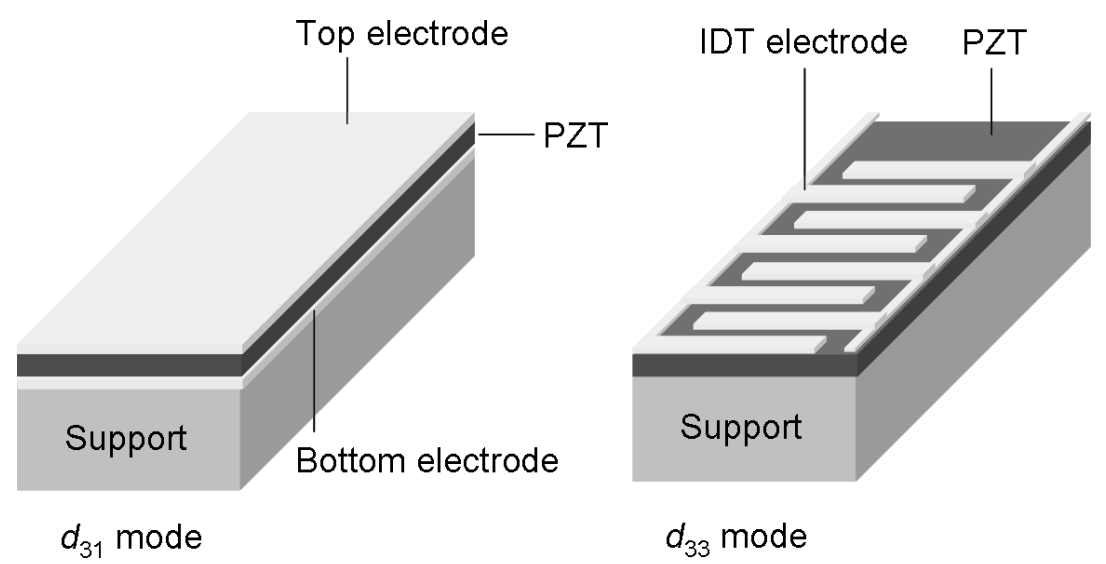

Figure 2.1 Electrode configurations for two common modes utilized for PZT transducers.

It is important to note that in polycrystalline PZT films, the polarization is randomly or slightly oriented. Their domains have to be aligned close to one direction by applying an electric field on the films in order to exhibit a net piezoelectric effect. Consequently, the polarization vector can be reoriented along different directions, allowing polycrystalline PZT films to utilize either $d_{31}$ or $d_{33}$ modes [31, 32]. In contrary, the polarization direction of the $\mathrm{ZnO}$ and AlN [33] or epitaxial PZT thin films is fixed by the crystal structure, and thus reorientation to other directions is not possible. In relaxor ferroelectric thin films like PMN-PT, the alignment of their domains depends on crystallographic orientation; thus it would be possible to enable their operation in $d_{31}$ or $d_{33}$ modes [34].

\subsection{Piezoelectric MEMS based on epitaxial PZT thin films}

Epitaxial PZT thin films grown on silicon substrates are receiving a lot of interest for many applications; especially their excellent properties are promising for the realization of high performance MEMS devices. The integration of high-quality epitaxial PZT thin films on silicon substrates can be achieved through a buffer layer based on epitaxial oxide thin films. Ramesh et al. reported the use of YSZ as a buffer layer, which can improve the remanent polarization with excellent fatigue, retention, and aging characteristics [35]. Dekkers et al. has also achieved the crystalline growth of PZT (001)-oriented thin films on silicon using a YSZ buffer layer [36]. Nevertheless, the structure of YSZ is complex and a relatively simple structure is desired. This layer can be replaced with a single perovskite STO buffer layer, which appears to be an excellent material as it exhibits a very high structural quality and can be grown epitaxially on silicon by molecular-beam epitaxy (MBE) [37]. When a high quality perovskite-terminated silicon substrate is obtained, epitaxial growth of other perovskite and related materials is facilitated. Conductive perovskite laysers such as $(\mathrm{La}, \mathrm{Sr}) \mathrm{CoO}_{3}$ or $\mathrm{SrRuO}_{3}$ can be used as a bottom electrode that constitutes also an epitaxial layer for 
successive growth of the epitaxial PZT thin film [38]. The PZT layers epitaxially grown on the aforementioned structure have shown to have improved piezoelectric properties [23, 39].

In spite of this advancement, the research focused on piezoelectric MEMS devices based on epitaxial PZT thin films has been seldom reported. Reilly et al. first reported on vibrational energy harvesters based on epitaxial thin films grown by pulsed laser deposition [40]. Despite the high quality of the epitaxial films, the power was significantly low due to some damages caused to the epitaxial PZT film during the fabrication process. Moreover, the devices in that work were fabricated on chip-scale silicon substrates. Nguyen et al. successfully demonstrated the epitaxial PZT cantilevers with conductive oxide SRO electrodes, achieving a long-term stability and reliability of the devices, but the fabrication throughput was significantly limiting as the cantilevers were fabricated on $20 \mathrm{~mm}$ $\times 20 \mathrm{~mm}$ SOI substrates [41]. Recently, Morimoto et al. designed and fabricated energy harvesters composed of epitaxial PZT films transferred onto stainless steel cantilevers to enhance output power efficiency and to improve structural toughness [42]. However, the fabrication throughput, reproducibility and device miniaturization seem to be limited; and the epitaxial PZT films were not directly grown on silicon substrates, but on $\mathrm{MgO}$ substrates. Therefore, two major challenges still remain for the realization of piezoelectric MEMS based on epitaxial PZT thin films: the control of the deposition of epitaxial PZT and related oxide thin films on silicon at the wafer-scale level; and the need of dedicated microfabrication processes for the epitaxial oxide thin films with a specific attention in maintaining the piezoelectric properties of the epitaxial PZT films. 


\section{References}

[1] Trolier-McKinstry S and Muralt P 2004 Thin film piezoelectrics for MEMS J. Electroceram. 12 7-17

[2] Bassiri-Gharb N 2008 Piezoelectric and acoustic materials for transducer applications (Springer) p 415

[3] Uozumi K, Ohsone K and White R M 1983 Generation and detection of ultrasonic lamb waves in a thin deposited film using interdigital transducers Appl. Phys. Lett. 43 917-9

[4] Tadigadapa S and Mateti K 2009 Piezoelectric MEMS sensors: state-of-the-art and perspectives Meas. Sci. Technol. 20092001

[5] Dubois M A and Muralt P 1999 Properties of aluminum nitride thin films for piezoelectric transducers and microwave filter applications Appl. Phys. Lett. 74 3032-4

[6] Vellekoop M J, Visser C C O, Sarro P M and Venema A 1990 Compatibility of zinc oxide with silicon IC processing Sens. Actuators, A 23 1027-30

[7] Hata T, Kawagoe S, Zhang W, Sasaki K and Yoshioka Y 1998 Proposal of new mixture target for PZT thin films by reactive sputtering Vacuum $\mathbf{5 1}$ 665-71

[8] Wang C and Kryder M H 2009 Low fatigue in epitaxial $\mathrm{Pb}\left(\mathrm{Zr}_{0.2} \mathrm{Ti}_{0.8}\right) \mathrm{O}_{3}$ on Si substrates with $\mathrm{LaNiO}_{3}$ electrodes by RF sputtering J. Electron. Mater. 38 1921-5

[9] Bouregba R, Sama N, Soyer C and Remiens D 2009 Analysis of size effects in $\mathrm{Pb}\left(\mathrm{Zr}_{0.54} \mathrm{Ti}_{0.46}\right) \mathrm{O}_{3}$ thin film capacitors with platinum and $\mathrm{LaNiO}_{3}$ conducting oxide electrodes J. Appl. Phys. 106044101

[10] Morita T, Wagatsuma Y, Cho Y, Morioka H, Funakubo H and Setter N 2004 Ferroelectric properties of an epitaxial lead zirconate titanate thin film deposited by a hydrothermal method below the Curie temperature Appl. Phys. Lett. 84 5094-6

[11] Feigl L, Zheng S J, Birajdar B I, Rodriguez B J, Zhu Y L, Alexe M and Hesse D 2009 Impact of high interface density on ferroelectric and structural properties of $\mathrm{PbZr}_{0.2} \mathrm{Ti}_{0.8} \mathrm{O}_{3} / \mathrm{PbZr}_{0.4} \mathrm{Ti}_{0.6} \mathrm{O}_{3}$ epitaxial multilayers J. Phys. D: Appl. Phys 42085305

[12] Walker D, Thomas P A and Collins S P 2009 A comprehension investigation of the structural properties of ferroelectric $\mathrm{PbZr}_{0.2} \mathrm{Ti}_{0.8} \mathrm{O}_{3}$ thin film grown by PLD Phys. Status Solidi A 206 1799-803

[13] Schneller $\mathrm{T}$ and Waser $\mathrm{R} 2007$ Chemical modifications of $\mathrm{Pb}\left(\mathrm{Zr}_{0.3} \mathrm{Ti}_{0.7}\right) \mathrm{O}_{3}$ precursor solutions and their influence on the morphological and electrical properties of the resulting thin films $J$. Sol-Gel Sci. Technol. 42 337-52

[14] Bhaskar A, Chang $\mathrm{T}-\mathrm{H}$, Chang $\mathrm{H}-\mathrm{Y}$ and Cheng $\mathrm{S}-\mathrm{Y} 2009 \mathrm{~Pb}\left(\mathrm{Zr}_{0.53} \mathrm{Ti}_{0.47}\right) \mathrm{O}_{3}$ thin films with different thickness obtained at low temperature by microwave irradiation Appl. Surf. Sci. 255 3795-800 
[15] Vu H N, Le M V, Bui H T and Nguyen M D 2009 Improvement of electrical property for $\mathrm{Pb}\left(\mathrm{Zr}_{0.53} \mathrm{Ti}_{0.47}\right) \mathrm{O}_{3}$ ferroelectric thin film deposited by sol-gel method on SRO electrode $J$. Phys.: Conf. Ser. 187012063

[16] Pan C Y, Chen Y -L and Tsai D S 2002 Synthesis and properties of lead zirconate titanate thin films via metalorganic chemical vapor deposition J. Mater. Res. 17 1536-42

[17] Otani Y, Okamura S and Shiosaki T 2004 Recent developments on MOCVD of ferroelectric thin films $J$. Electroceram. 13 15-22

[18] Ruby R 2007 Review and comparison of bulk acoustic wave FBAR, SMR technology Proc. IEEE Ultrasonics Symp. 7 1029-40

[19] Lee $\mathrm{H}-\mathrm{C}$ and Lee $\mathrm{W}-\mathrm{J} 2002$ Characterization of $\mathrm{Pb}\left(\mathrm{Zr}, \mathrm{Ti}^{2} \mathrm{O}_{3}\right.$ thin films fabricated by plasma enhanced chemical vapor deposition on Ir-based electrodes J. Vac. Sci. Technol. A 20 193947

[20] Setter N, Damjanovic D, Eng L, Fox G, Gevorgian S, Hong S, Kohlstedt H, Park N Y, Stephenson G B, Stolitchnov I, Taganstev A K, Taylor D V, Yamada T and Streiffer S 2006 Ferroelectric thin films: review of materials, properties, and applications J. Appl. Phys. 100 051606

[21] Setter N 2005 Electroceramics-based MEMS: fabrication-technology, and applications Electronic Materials: Science and Technology ed H L Tuller (New York: Springer)

[22] Muralt P 2000 Ferroelectric thin films for micro-sensors and actuators: a review $J$. Micromech. Microeng. 10 136-46

[23] Wang Y, Ganpule C, Liu B T, Li H, Mori K, Hill B, Wuttig M, Ramesh R, Finder J, Yu Z, Droopad R and Eisenbeiser K 2002 Epitaxial ferroelectric $\mathrm{Pb}(\mathrm{Zr}, \mathrm{Ti}) \mathrm{O}_{3}$ thin films on Si using $\mathrm{SrTiO}_{3}$ template layers Appl. Phys. Lett. 80 97-9

[24] Rabe K, Ahn C H and Triscone J -M 2007 Physics of ferroelectrics a modern perspective (Springer) p 219

[25] Detalle M, Wang G, Remiens D, Ruterana P, Roussel P and Dkhil B 2007 Comparison of structural and electrical properties of PMN-PT films deposited on Si with different bottom electrodes J. Cryst. Growth 305 137-43

[26] Damjanovic D, Muralt P and Setter N 2001 Ferroelectric sensors IEEE Sens. J. 1 191-206

[27] Muralt P 2008 Recent progress in materials issues for piezoelectric MEMS J. Am. Ceram. Soc. 91 1385-96

[28] Elfrink R, Kamel T M, Goedbloed M, Matova S, Hohlfeld D, van Andel Y and van Schaijk R 2009 Vibration energy harvesting with aluminum nitride-based piezoelectric devices $J$. Micromech. Microeng. 19094005

[29] Xu F, Wolf R A, Yoshimura T and Trolier-McKinstry 2002 Piezoelectric films for MEMS applications $11^{\text {th }}$ International Symposium on Electrets $\mathrm{p} 386-96$ 
[30] Xu B, Polcawich R G, Trolier-McKinstry S, Ye Y, Cross L E, Bernstein J J and Miller R 1999 Sensing characteristics of in-plane polarized lead zirconate titanate thin films Appl. Phys. Lett. 75 4180-2

[31] Muralt P, Marzencki M, Belgacem B, Calame F and Basrour S 2009 Vibration energy harvesting with PZT micro device Procedia Chemistry 1 1191-4

[32] Zhang Q Q, Gross S J, Tadigadapa S, Jackson T N, Djuth F T and Trolier-McKinstry S 2003 Lead zirconate titanate films for $d_{33}$ mode cantilever actuators Sens. Actuators, A 105 91-7

[33] Hong E, Krishnaswamy S V, Freidhoff C B and Trolier-McKinstry S 2005 Micromachined piezoelectric diaphragms actuated by ring shaped interdigitated transducer electrodes Sens. Actuators, A 119 520-6

[34] Eom C B 2006 personal communication

[35] Ramesh R, Gilchrist H, Sands T, Keramidas V G, Haakenaasen R and Fork D K 1993 Ferroelectric $\mathrm{La}-\mathrm{Sr}-\mathrm{Co}-\mathrm{O} / \mathrm{Pb}-\mathrm{Zr}-\mathrm{Ti}-\mathrm{O} / \mathrm{La}-\mathrm{Sr}-\mathrm{Co}-\mathrm{O}$ heterostructures on silicon via template growth Appl. Phys. Lett. 63 3592-4

[36] Dekkers M, Nguyen M D, Steenwelle R, te Riele P M, Blank D H A and Rijnders G 2009 Ferroelectric properties of epitaxial $\mathrm{Pb}(\mathrm{Zr}, \mathrm{Ti}) \mathrm{O}_{3}$ thin films on silicon by control of crystal orientation Appl. Phys. Lett. 95012902

[37] McKee R A, Walker F J and Chisholm M F 1998 Crystalline oxides on silicon: The first five monolayers Phys. Rev. Lett. 81 3014-17

[38] Ramesh R and Schlom D G 2002 Orienting Ferroelectric Films Science 296 1975-1976

[39] Lin A, Hong X, Wood V, Verevkin A A, Ahn C H, McKee R A, Walker F J and Specht E D 2001 Epitaxial growth of $\mathrm{Pb}\left(\mathrm{Zr}_{0.2} \mathrm{Ti}_{0.8}\right) \mathrm{O}_{3}$ on $\mathrm{Si}$ and its nanoscale piezoelectric properties Appl. Phys. Lett. 78 2034-36

[40] Reilly E K and Wright P K 2009 Modeling, fabrication and stress compensation of an epitaxial thin film piezoelectric microscale energy scavenging device J. Micromech. Microeng. 19095014

[41] Nguyen M D, Nazeer H, Karakaya K, Pham S V, Steenwelle R, Dekkers M, Abelmann L, Blank D H A and Rijnders G 2010 Characterization of epitaxial $\mathrm{Pb}(\mathrm{Zr}, \mathrm{Ti}) \mathrm{O}_{3}$ thin films deposited by pulsed laser deposition on silicon cantilevers J. Micromech. Microeng. 20 085022

[42] Morimoto K, Kanno I, Wasa Kiyotaka and Kotera H 2010 High-efficiency piezoelectric energy harvesters of $c$-axis-oriented epitaxial PZT films transferred onto stainless steel cantilevers Sens. Actuators, A 163 428-32 



\title{
Chapter 3
}

\section{Epitaxial piezoelectric MEMS on silicon}

\author{
D. Isarakorn', A. Sambri², P. Janphuang ${ }^{1}$, D. Briand 1 , S. Gariglio², J. -M. Triscone², F. Guy ${ }^{3}$, \\ J. W. Reiner ${ }^{4}$, C. H. Ahn ${ }^{4}$ and N. F. de Rooij ${ }^{1}$ \\ ${ }^{1}$ École Polytechnique Fédérale de Lausanne (EPFL), Institute of Microengineering (IMT), \\ The Sensors, Actuators and Microsystems Laboratory (SAMLAB), \\ Rue Jaquet-Droz 1, P.O. Box 526, 2002 Neuchâtel, Switzerland \\ ${ }^{2}$ Department of Condensed Matter Physics, University of Geneva, \\ 24 quai Ernest-Ansermet, 1211 Geneva 4, Switzerland \\ ${ }^{3}$ TIN, HEPIA, 4 Rue de la Prairie, CH-1202 Geneva, Switzerland \\ ${ }^{4}$ Department of Applied Physics, Yale University, Becton Center, \\ P.O. Box 208284 New Haven, CT 06520-8284 USA
}

\begin{abstract}
This paper reports on the microfabrication and characterization of piezoelectric MEMS structures based on epitaxial $\mathrm{Pb}\left(\mathrm{Zr}_{0.2} \mathrm{Ti}_{0.8}\right) \mathrm{O}_{3}$ (PZT) thin films grown on silicon wafers. Membranes and cantilevers are realized using a sequence of microfabrication processes optimized for epitaxial oxide layers. Different issues related to the choice of materials and to the influence of the fabrication processes on the properties of the piezoelectric films are addressed. These epitaxial PZT transducers can generate relatively large deflections at low bias voltages in the static mode. Estimations of the piezoelectric coefficient $d_{31}$ of the epitaxial PZT thin film $(100 \mathrm{~nm})$ yield $130 \mathrm{pm} \mathrm{V}^{-1}$. In the dynamic mode, the performance of the epitaxial PZT transducers in terms of the resonant frequency, modal shape, and quality factor are examined. An epitaxial PZT/Si cantilever $\left(1000 \times 2500 \times 40 \mu^{3}\right)$ resonating in air and in vacuum exhibits a deflection of several microns with quality factors of 169 and 284 , respectively. For a $1500 \mu \mathrm{m}$ diameter membrane, the quality factor is 50 at atmospheric pressure, and this rises to 323 in a pressure of 0.1 mbar. These results indicate the high potential of epitaxial piezoelectric MEMS, which can impact a variety of technological applications.
\end{abstract}

Published in Journal of Micromechanics and Microengineering, Vol. 20, 055008, 2010 


\subsection{Introduction}

Piezoelectric thin films have attracted attention because of the possibility of various applications in microelectronics and microelectromechanical systems (MEMS) such as nonvolatile memories, microsensors and microactuators. Among piezoelectric materials, $\mathrm{Pb}(\mathrm{Zr}, \mathrm{Ti}) \mathrm{O}_{3}$ is of primary interest due to its outstanding piezoelectric properties compared to $\mathrm{ZnO}$ and AlN. However, for MEMS fabrication, PZT has to be prepared as a thin film on a silicon substrate. This step often leads to a degradation of the piezoelectric properties due to inferior surface quality [1]. To improve the properties of the PZT thin films, research has been focused on the crystallization and orientation of these films and on the optimization of the interface between the film and the electrode.

The growth of epitaxial piezoelectric (epi-piezo) thin films on silicon has been studied extensively over the past decade [2-5], since it is a possible way to integrate high quality piezoelectric films on silicon.

Epitaxial films in general exhibit properties, including piezoelectric coefficients, polarization, and dielectric constant superior to polycrystalline films [6]. We have demonstrated that epi-piezo thin films can be grown on (001) silicon through oxide transition layers. These epi-piezo films are smooth on a nanometric scale and exhibit microstructural homogeneities, and very large piezoelectric coefficients [5]. Taking these results into account, we can expect several advantages from piezoelectric MEMS devices based on epi-piezo thin films such as high forces at low operational voltage, high frequency operation and high energy-conversion efficiency. Moreover, the epitaxial growth of piezoelectric thin films on oxide layers can reduce the fatigue effect and cyclic depolarization [7], a reduction that is necessary for reliable use in devices. In order to obtain high quality epi-piezo thin films on silicon, it is essential to develop a controlled growth procedure, and a microfabrication process suitable for the piezoelectric epitaxial layer. However, few studies have investigated the microfabrication process of epi-piezo MEMS on STO substrates and on silicon substrates [8-10]. It has been shown that the development of appropriate fabrication processes to realize micromachined structures is a crucial technological step towards the development of novel MEMS devices [11-16].

In this paper, we report on the microfabrication of epi-piezo MEMS membranes and cantilevers and their mechanical characteristics. We describe the growth and the structural characterization of epi-piezo films grown on silicon wafers through oxide transition layers. These piezoelectric films are very smooth with rms roughness of about $5 \mathrm{~nm}$ over $1 \mu \mathrm{m}^{2}$ and exhibit high crystalline quality. A selection of microfabrication processes with associated process flows for the fabrication of epi-piezo MEMS is highlighted. We focus here on the microfabrication techniques required to pattern these epitaxial thin films and especially on the influence of the processing temperature and of the type of materials used as top electrodes on the epi-piezo film properties. The characterization of the mechanical properties of these epi-piezo MEMS devices, performed by optical techniques, reveals their high performance for actuation in static and dynamic modes. 


\subsection{Epitaxial thin films growth on silicon}

For growing epitaxial perovskite thin films with robust piezoelectric response on silicon, proper intermediate layers are required. Indeed, in order to deal with the differences in bonding, chemistry, and coordination between silicon and the oxide layer, McKee et al. [17] developed a procedure for the growth of an epitaxial $\mathrm{SrTiO}_{3}$ (STO) layer directly on silicon. In our work, a 10-20 unit-cell thick STO buffer layer is grown onto 2" $\mathrm{Si}$ (001) wafers by MBE [18]. Successively, we grow by off-axis magnetron sputtering a $\mathrm{SrRuO}_{3}$ (SRO) film used as a bottom electrode and then a ferroelectric $\mathrm{Pb}\left(\mathrm{Zr}_{0.2} \mathrm{Ti}_{0.8}\right) \mathrm{O}_{3}$ layer [19]. $\mathrm{Pb}\left(\mathrm{Zr}_{0.2} \mathrm{Ti}_{0.8}\right) \mathrm{O}_{3}$ was chosen for its good lattice match with STO and SRO. The typical final stack results as PZT $(100 \mathrm{~nm}) / \mathrm{SRO}(30 \mathrm{~nm}) / \mathrm{STO}(6 \mathrm{~nm}) / \mathrm{Si}$.

$\mathrm{X}$-ray $\theta-2 \theta$ diffractograms display only ( $00 l)$ peaks, confirming the $c$-axis orientation of the oxide stack and revealing a PZT $c$-axis lattice parameter of $4.13 \AA$ (figure 3.1(a)). Finite size oscillations around the SRO (001) and (002) reflections attest of the high crystalline coherence of the bottom electrode. Rocking curve measurements reveal the good crystalline quality of the piezoelectric layer with a full width half maximum (FWHM) of $0.5^{\circ}$ around the PZT (001) peak. The FWHM of Si (004) rocking curve is around $0.01^{\circ}$. Detailed diffraction analyses (figure 3.1(b)) confirm the epitaxial relationship between the oxide layers and the substrate: PZT[001]/SRO[001]/STO[001]/Si[001] and $\mathrm{PZT}[100] / \mathrm{SRO}[100] / \mathrm{STO}[100] / \mathrm{Si}[110]$. Local measurements of the $d_{33}$ piezoelectric coefficient, performed with an atomic force microscope [20], reveal the ferroelectric behavior of the PZT layer: the estimated piezoelectric coefficient $d_{33}$ is $50 \mathrm{pm} \mathrm{V}^{-1}$. The current-voltage loop and the corresponding polarization were determined using a ferroelectric tester TF analyzer 2000 system on capacitors with $\mathrm{Cr} / \mathrm{Au}$ top electrodes of $100 \times 100 \mu \mathrm{m}^{2}$ area. Figure 3.2 shows a hysteresis loop, measured at $100 \mathrm{~Hz}$ on a $200 \mathrm{~nm}$ thick PZT layer. Ferroelectric switching peaks at a coercive voltage of $6 \mathrm{~V}$ are visible without any evidence of leakage currents up to $16 \mathrm{~V}$, indicating the robust dielectric properties of the epi-piezo thin film on silicon. The value of remnant polarization is estimated to be around $70 \mu \mathrm{C} \mathrm{cm}^{-2}$ and the coercive field about $300 \mathrm{kV} \mathrm{cm}^{-1}$. 

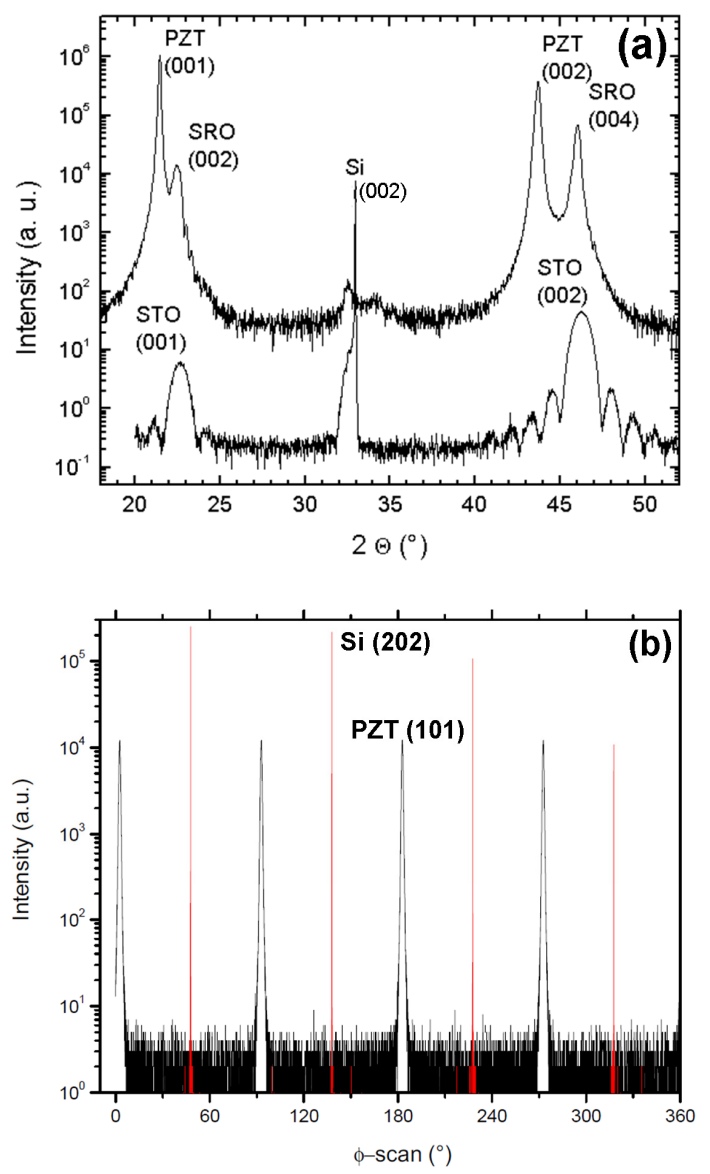

Figure 3.1. (a) $\theta-2 \theta$ diffractograms of a $\operatorname{PZT}(100 \mathrm{~nm}) / \mathrm{SRO}(30 \mathrm{~nm})$ heterostructure (top), $\theta-2 \theta$ diffractograms of the STO buffer layer on silicon (001) (bottom). (b) $\phi$-scan measurements of the PZT (101) and Si (202) reflections.

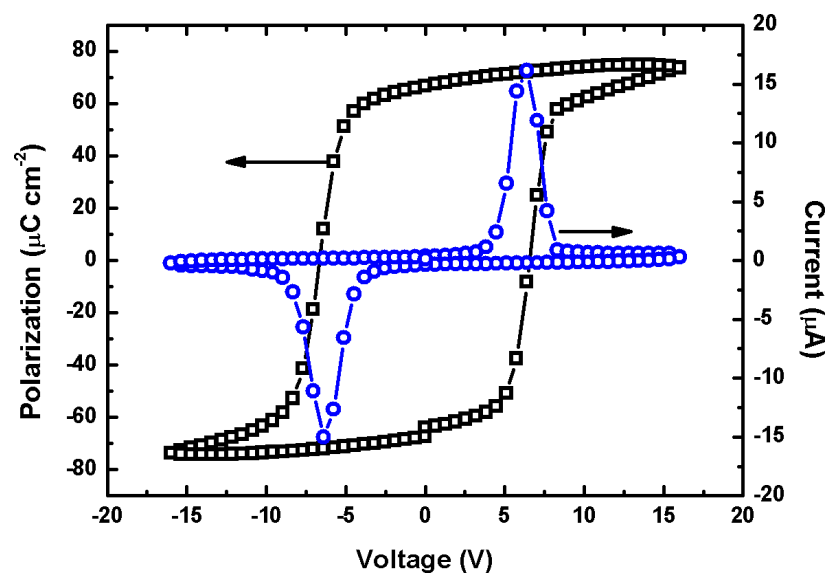

Figure 3.2. Current (right axis, round points)-voltage loop and corresponding polarization hysteresis of an epitaxial PZT thin film (200 nm thick) on silicon measured at $100 \mathrm{~Hz}$. 


\subsection{Microfabrication}

Epitaxial piezoelectric MEMS are based on a piezoelectric layer grown on a silicon substrate through two oxide layers: STO used as buffer and metallic SRO used as bottom electrode. The presence of such layers requires specific microfabrication processes for their patterning. Moreover, it is essential that the excellent properties of the epi-piezo film are retained after the whole sequence of microfabrication processes to maximize the performance of fabricated devices. The process we have developed has been optimized with the right choice of the top electrode material and the minimization of the thermal budget. The variation in the polarization during the progress of the microfabrication process was also investigated.

\subsubsection{Top electrode materials}

In general, top electrodes are deposited and patterned at the beginning of the fabrication process to protect the piezoelectric thin films during the processing. However, it has been reported that in such heteostructures the thermal load due to the various step of the microfabrication can strongly affect the ferroelectric properties [21]. We have thus investigated the thermal budget for epitaxial PZT thin films with different top electrode materials. Tests were on ferroelectric layers grown on $10 \times 10 \mathrm{~mm}^{2}$ STO substrates. The capacitor structure consists of a SRO bottom electrode (30 nm), a PZT $100 \mathrm{~nm}$ thick film and a $60 \times 60 \mu \mathrm{m}^{2}$ top electrodes deposited at room temperature through a shadow mask by an e-beam evaporator. The metals used as the top electrodes and their thicknesses are shown in table 3.1 .

Table 3.1. Top electrode materials and their thicknesses.

\begin{tabular}{lcc}
\hline Electrode type & Adhesive layer & Electrode material \\
\hline $\mathbf{A u} / \mathbf{C r}$ & Cr: $10 \mathrm{~nm}$ & Au: $100 \mathrm{~nm}$ \\
$\mathbf{P t} / \mathbf{T a}$ & Ta: $5 \mathrm{~nm}$ & Pt: $135 \mathrm{~nm}$ \\
$\mathbf{P t} / \mathbf{T i}$ & Ti: $5 \mathrm{~nm}$ & Pt: $135 \mathrm{~nm}$ \\
\hline
\end{tabular}

We measured current-voltage characteristics after annealing the sample in air for 30 minutes at different temperatures, ranging from 100 to $350{ }^{\circ} \mathrm{C}$. The results show that the degradation in the remanent polarization $(\mathrm{Pr})$ is most severe for the $\mathrm{PZT}$ thin films with a $\mathrm{Pt} / \mathrm{Ti}$ electrode, and least severe for the one with a $\mathrm{Au} / \mathrm{Cr}$ electrode. However, even for $\mathrm{Au} / \mathrm{Cr}$ electrodes annealed at $350{ }^{\circ} \mathrm{C}$, the leakage current becomes the dominant contribution.

On the contrary, ferroelectric properties of bare epitaxial PZT thin films without metal electrodes are stable up to temperatures as high as $735{ }^{\circ} \mathrm{C}$ [22]. In this regard, we measured I-V characteristics of test structures where the metallization was realized after the annealing at high temperature. In this case, the polarization is not degraded by the annealing up to $400{ }^{\circ} \mathrm{C}$. 
These tests show clearly that the top electrode materials induce damages to the PZT thin film during the thermal annealing Thus, in order to avoid degradation of the dielectric properties, the electrode materials have been deposited and patterned at the end of the fabrication process.

\subsubsection{Process flow}

Epitaxial PZT transducers (membranes and cantilevers) are fabricated using micro-patterning techniques optimized for these oxide layers deposited on silicon wafers. The process flow used to realize these structures is illustrated in figure 3.3. Starting from a heterostructure of PZT/SRO/STO grown on $\mathrm{Si}$ as described in the previous section (figure 3.3(a)), contact pads are opened through the epitaxial PZT film to give access to the SRO bottom electrode, as shown in figure 3.3(b). For this step, a combination of $150 \mathrm{HCl}: 350 \mathrm{H}_{2} \mathrm{O}: 1 \mathrm{HF}$ solution at $55{ }^{\circ} \mathrm{C}$ is used with 6 - $\mu \mathrm{m}$-thick AZ4562 photoresist as the etching mask. The etch rate is of about $40 \mathrm{~nm} \mathrm{~s}^{-1}$. The use of thick photoresist is designed to reduce heavy under etching. This etch recipe is an effective technique to pattern large areas of PZT due to high etch rate, low cost and selective to the SRO metallic oxide electrode compared to a $\mathrm{HCl}: \mathrm{NHO}_{3}: \mathrm{H}_{2} \mathrm{O}$ solution [23].

(a)

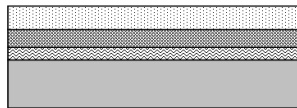

(b)

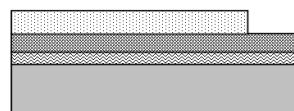

(c)

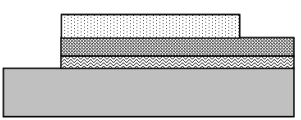

(d)

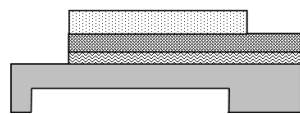

(e)

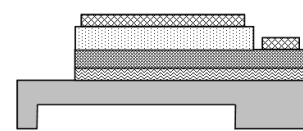

(f)

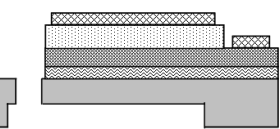

TO
Silicon

Figure 3.3. Schematic of the microfabrication process for epi-piezo MEMS structures.

For the cantilever fabrication, the shape of the cantilevers must be patterned from the top side through the PZT/SRO/STO stack while no top side structuring is required for the membranes. We investigated the etching of the multilayer stack in a single processing step using ion milling. The multilayer stack is etched using an Ion Tech ion milling machine with a pressure of $5.4 \times 10^{-4} \mathrm{mbar}$ and a $27 \mathrm{~mA}$ beam current with a bias voltage of $350 \mathrm{~V}$ (figure 3.3(c)). Materials like Pt or Au can be used as hard masks in the ion milling process and at the same time they can serve as top electrodes later $[24,25]$. However we found that these materials can degrade the ferroelectricity in epitaxial piezoelectric films during high temperature processing as discussed in previous subsection. For this reason, the patterning of this stack was carried out by using the 11- $\mu$ m-thick AZ4562 photoresist as a mask. Due to significant increase in temperature during the ion milling process, the photoresist was 
hardened and its stripping became extremely difficult. Therefore, an ion milling process with alternating etching and cooling steps to avoid rising temperature was used. The alternative etching and cooling sequences were done for $3 \mathrm{~min}$ and $2 \mathrm{~min}$, respectively, for a total etching time of $20 \mathrm{~min}$. In this case, the photoresist can be removed easily by an oxygen plasma etcher followed by acetone. The PZT $(100 \mathrm{~nm}) / \mathrm{SRO}(30 \mathrm{~nm}) / \mathrm{STO}(6 \mathrm{~nm})$ stack etched by the ion milling shows steep etched profiles without residues. However, since the ion milling etching method does not exhibit an etching selectivity to the materials underneath, the etching progress must be monitored carefully since there is no etch-stop mechanism. It should be noted that this multilayer oxide stack can be structured by a $\mathrm{CHF}_{3} / \mathrm{Ar}$ plasma using the molybdenum (Mo) mask [26]. However it could have some influence on the degradation of the ferroelectric properties of epitaxial piezoelectric thin films.

The back side of the wafer is then etched by deep reactive ion etching (DRIE) to define the thickness of the silicon membrane/cantilever (figure 3.3(d)). An $\operatorname{Au}(100 \mathrm{~nm}) / \mathrm{Cr}(10 \mathrm{~nm})$ layer is evaporated for electrical contacts to the top and bottom electrodes by a lift-off process (figure 3.3(e)). We deposited and patterned the electrodes at the end of the fabrication process in order to minimize the thermal budget during the process to avoid the degradation of the ferroelectric layer [27]. At this step, membranes are obtained. To release the cantilevers from the silicon wafer, a structural release process is performed using frequency-tripled Nd:YAG laser $(\lambda=355 \mathrm{~nm})$ micromachining, which is operated in the direct write approach with a beam diameter of $20 \mu \mathrm{m}$ from the frontside of the wafer (figure 3.3(f)). This technique offers an advantage for a structural release process of fragile structures from the top side of the wafer because it does not require a photolithographic step [28-30].

In order to optimize the microfabrication process, the value of the PZT remanent polarization is evaluated after each processing step. Figure 3.4 shows the P-E loops after the metallization process. As can be seen, no marked change in the expitaxial PZT thin film polarization is observed after the process. However, we observe leakage currents on some devices when an $\mathrm{AC}$ voltage over $10 \mathrm{~V}$ is applied, and we noticed that the coercive voltage slightly decreased.

Circular membranes fully covered by an electrode with different diameters of 1000, 1500 and $2000 \mu \mathrm{m}$ with a silicon thickness of $20 \mu \mathrm{m}$, and cantilever beams $\left(1000 \times 2500 \times 40 \mu \mathrm{m}^{3}\right)$ have been fabricated. Some of the fabricated epitaxial PZT transducers are shown in figure 3.5. The dimensions of these devices are based primarily on our specific needs aiming at developing and microfabricating a cantilever-based vibration energy scavenger and a membrane-based biosensor. For clarity, this paper will only discuss the behaviors and performances of the devices in static and dynamic operations. Researches on the specific applications will be reported elsewhere. 


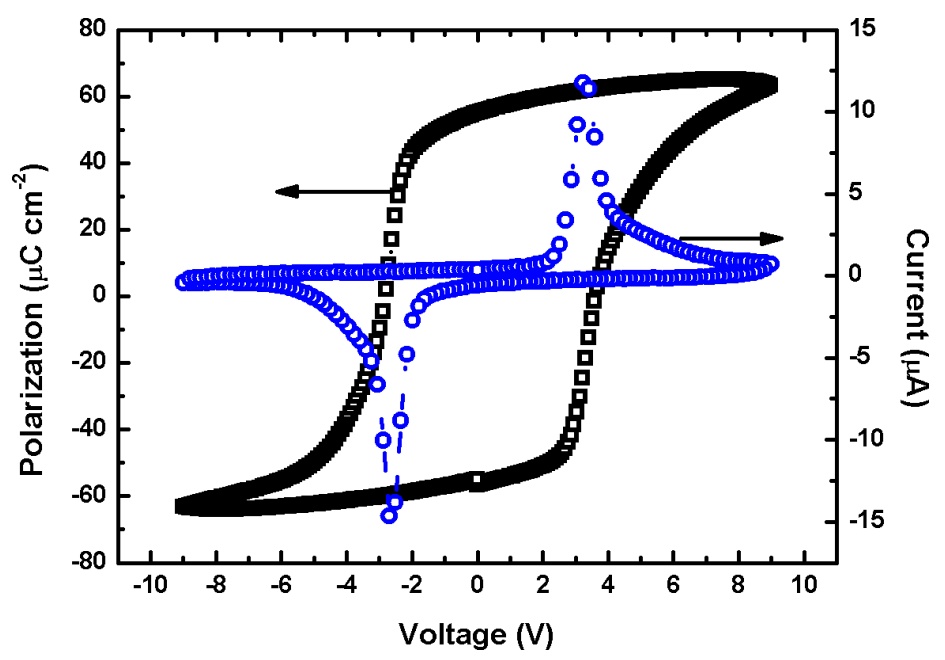

Figure 3.4. P-E loop and corresponding current-voltage loop of epitaxial PZT devices after the metallization process.
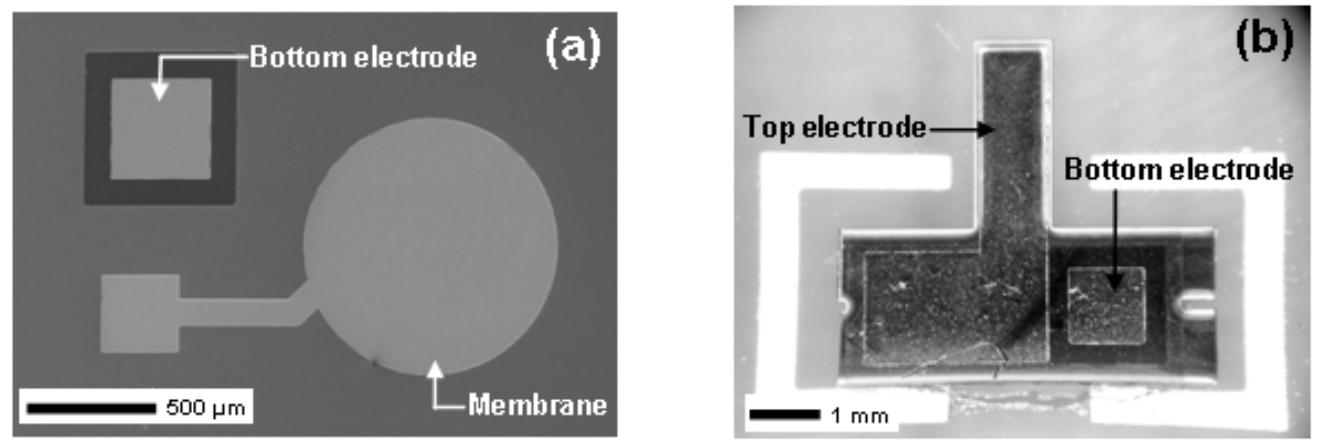

Figure 3.5. (a) SEM image of a circular membrane (top view) with a diameter of $1000 \mu \mathrm{m}$. (b) Optical image of a cantilever $\left(1000 \times 2500 \times 40 \mu \mathrm{m}^{3}\right)$.

\subsection{Results and discussion}

\subsubsection{Static behavior}

We have studied the static behavior of the membranes. A DC bias voltage in the range of 0 to $4 \mathrm{~V}$ is applied between the top and bottom electrodes, and the deflection on the entire surface of the membrane is measured using an interferometric profiler (Wyko NT1100). It should be noted that high leakage currents are observed on some devices at the DC bias voltage over $4 \mathrm{~V}$. This is possibly due to the large area of the contacts shown in figure 3.5. Therefore, the bias voltage up to $4 \mathrm{~V}$ is the upper limit for all static measurements. 
The surface and deflection profiles of a $2000 \mu \mathrm{m}$ diameter membrane are shown in figure 3.6. Without applying a bias voltage, the downward bending of the membrane due to residual stress is around $\sim 6 \mu \mathrm{m}$. The residual stress present in the epitaxial films after growth was evaluated as low as $60 \mathrm{MPa}$ using a thin film stress measurement system (Tencor FLX-2320A). Such a small level of stress has been demonstrated by E. Hong et al. [31] to not be the cause of the bending observed. Thus, the small bending of epitaxial structures may be due to the presence of residual-stress built in by the large area of the $\mathrm{Au} / \mathrm{Cr}$ electrode layers. Applying a DC bias voltage of $4 \mathrm{~V}$, the membrane flexed upward, resulting in a deflection of about $27 \mu \mathrm{m}$ at its center. Figure 3.7 shows the center deflections of membranes with different diameters as a function of voltage. At the bias voltage of $4 \mathrm{~V}, 1000 \mu \mathrm{m}$ and $1500 \mu \mathrm{m}$ diameter membranes can generate the center deflections of around $1.2 \mu \mathrm{m}$ and $12 \mu \mathrm{m}$, respectively. Such membranes exhibit large defections at low voltages even if actuated by very thin epitaxial PZT films. These values are not directly comparable to the ones reported in the literatures due to different diameters, thicknesses and electrode configurations, but for example E. Hong et al. obtained the deflection of $7 \mu \mathrm{m}$ at $100 \mathrm{~V}$ for a $800 \mu \mathrm{m}$ diameter membrane with $2 \mu \mathrm{m}$ thick PZT and $2.8 \mu \mathrm{m}$ thick silicon [31], and a membrane of $2500 \mu \mathrm{m}$ in diameter with $2 \mu \mathrm{m}$ thick PZT and $15 \mu \mathrm{m}$ thick silicon generated $5.4 \mu \mathrm{m}$ deflection at $50 \mathrm{~V}$ reported by Y. Hishinuma et al. [32]. A clear advantage of our technology is that our membranes actuated by very thin epitaxial PZT films enable large defections at very low voltages, which is very promising for low voltage applications.

Static deflection of the $2500 \mu \mathrm{m}$ long cantilevers has also been performed by applying a DC bias voltage in the range of 0 to $4 \mathrm{~V}$. The upward bending of the beam due to residual stress is initially observed to be around $0.18 \mu \mathrm{m}$. The deflection of the free end of the beams as a function of the applied bias voltage is shown in figure 3.8. The PZT/Si cantilevers show non-linear displacement at a low bias voltage. However, the deflection of the beam exhibits excellent linear behavior when applying the bias voltage in the range of 1 to $4 \mathrm{~V}$. The maximum relative deflection is $\sim 4 \mu \mathrm{m}$ at $4 \mathrm{~V}$. In the linear domain ( 1 to $4 \mathrm{~V}$ ), the piezoelectric coefficient $d_{31}$ is estimated from the slope of the linear fit using the following equations [33]:

$$
\begin{gathered}
d_{31}=-\frac{1}{3} \frac{K}{s_{s} s_{p} h_{s}\left(h_{s}+h_{p}\right) L^{2}} \frac{\delta}{V} \\
K=4 s_{p} s_{s} h_{s} h_{p}^{3}+4 s_{p} s_{s} h_{s}^{3} h_{p}+s_{p}^{2} h_{s}^{4}+s_{s}^{2} h_{p}^{4}+6 s_{p} s_{s} h_{s}^{2} h_{p}^{2}
\end{gathered}
$$

where $s_{s}$ and $s_{p}$ are the compliance of the Si layer $\left(7.7 \times 10^{-12} \mathrm{~m}^{2} \mathrm{~N}^{-1}[34]\right)$ and PZT film $\left(12.4 \times 10^{-}\right.$ ${ }^{12} \mathrm{~m}^{2} \mathrm{~N}^{-1}[35]$ ) respectively, $\delta$ the measured deflection, $V$ the applied bias voltage, $L$ the cantilever length, $h_{s}$ the thickness of Si layer, and $h_{p}$ the thickness of the PZT film. Looking at formula (3.1) 
and (3.2), it can be seen that the main contribution comes from the terms involving the thickness of the $\mathrm{Si}\left(h_{s}\right)$ which is two orders of magnitude larger than the thickness of the other layers. For this reason, the contributions of STO, SRO and $\mathrm{Cr} / \mathrm{Au}$ are omitted in the calculation.
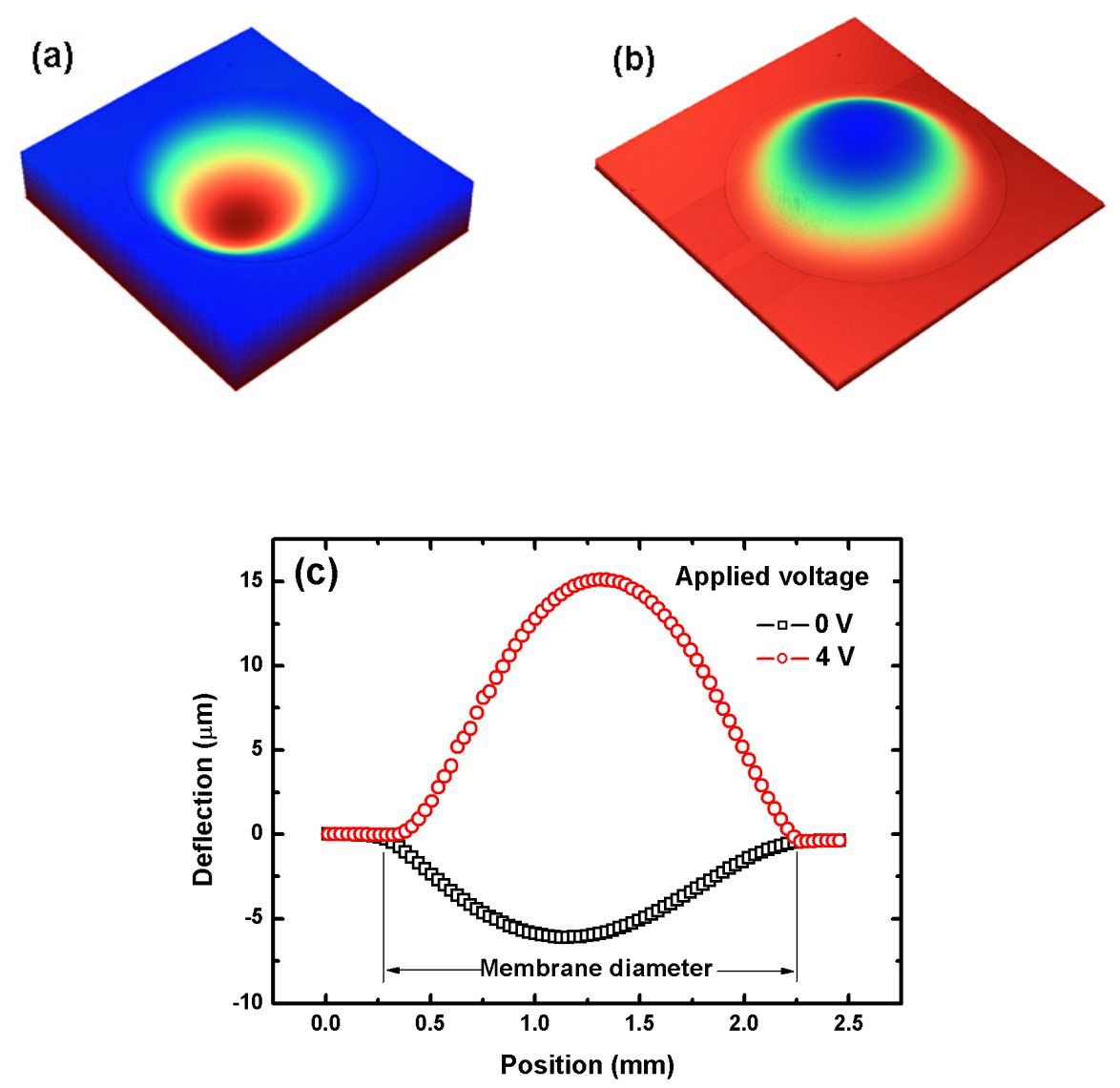

Figure 3.6. Three-dimensional plots of a $2000 \mu \mathrm{m}$ diameter membrane with bias voltage of (a) $0 \mathrm{~V}$ and (b) $4 \mathrm{~V}$ observed from the top side. (c) Deflection profiles of the membrane with different bias voltages. 


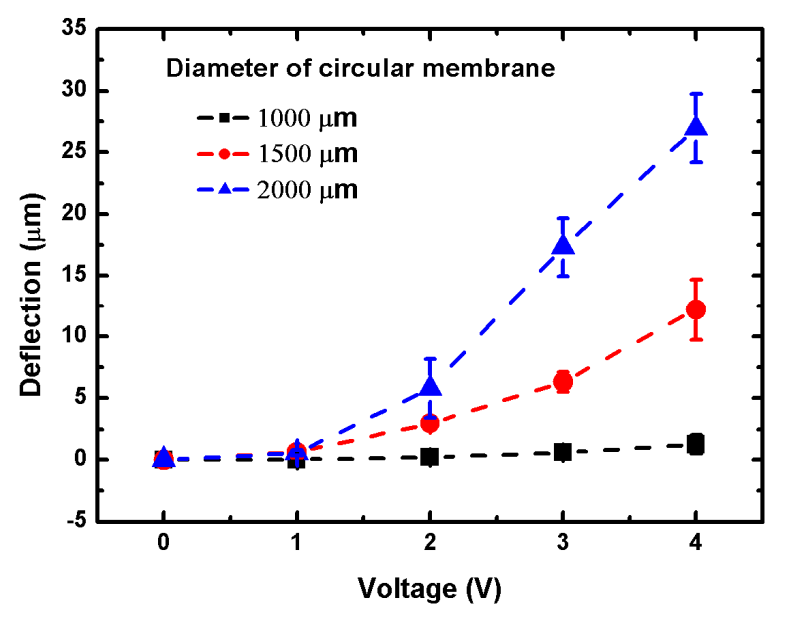

Figure 3.7. Center deflections of epitaxial PZT membranes with different diameters as a function of applied voltage.

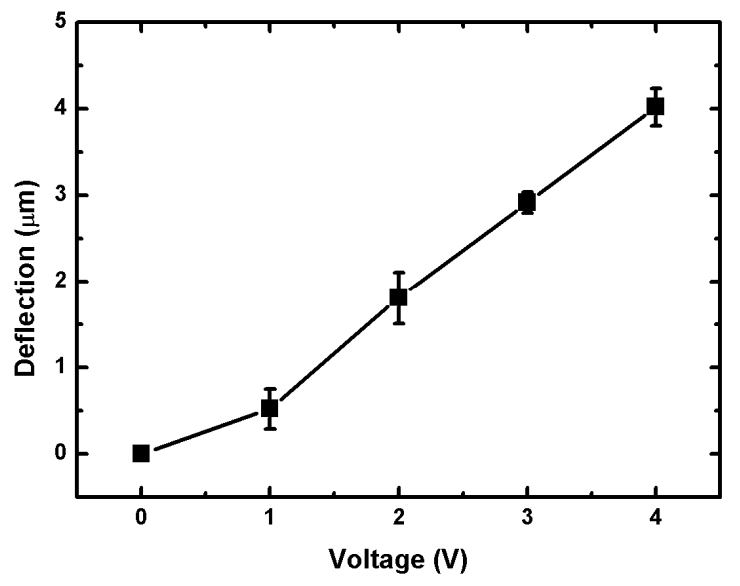

Figure 3.8. Deflection of the free end of the cantilever $\left(1000 \times 2500 \times 40 \mu \mathrm{m}^{3}\right)$ under various applied voltages.

Calculation of the piezoelectric coefficient $d_{31}$ corresponding to the range of 1 to $4 \mathrm{~V}$ of the applied bias voltages yields a value of $130-140 \mathrm{pm} \mathrm{V}^{-1}$, which is better than the value of polycrystalline films with the same $\mathrm{Zr}$ /Ti ratio [36]. The piezoelectric coefficient $d_{31}$ can be increased by increasing the PZT thickness as reported by Haccart et al [37]. Research on this topic is now underway to increase the piezoelectric coefficients of the epitaxial PZT thin films. 


\subsubsection{Dynamic behavior}

The dynamic behavior is another important parameter to consider for PZT transducers. The resonant frequencies and modal shapes of transducers are characterized using a Polytec MVS-400 laser Doppler vibrometer. A large range of frequencies is spanned to find the first five modes of resonances. Modal shapes of each resonant mode are also characterized. Quality factors of transducers operating in atmospheric pressure and under vacuum are evaluated using a Polytec OFV 502 fiber interferometer and OFV 3000 vibrometer controller.

The membranes are excited by a white noise signal with an amplitude of $1 \mathrm{~V}$ in the frequency range of 0 to $400 \mathrm{kHz}$. The laser beam is positioned slightly off-centered from the membrane center in order to detect both symmetric and asymmetric modes of vibrations. In figure 3.9, the frequency response of a $2000 \mu \mathrm{m}$ diameter membrane is presented. In order to determine the right resonant frequencies, excluding the response peaks due to environmental inputs, the modal shapes of vibrations are characterized by means of laser scanning while applying a $1 \mathrm{~V}$ sinusoidal signal. Figure 3.10 shows the modal shapes corresponding to the first five resonant frequency of the $2000 \mu \mathrm{m}$ diameter membrane. The first resonance appeared at $71.56 \mathrm{kHz}$, the second at $143.75 \mathrm{kHz}$, the third at 245.00 $\mathrm{kHz}$, the forth at $276.88 \mathrm{kHz}$, and the fifth at $343.44 \mathrm{kHz}$.

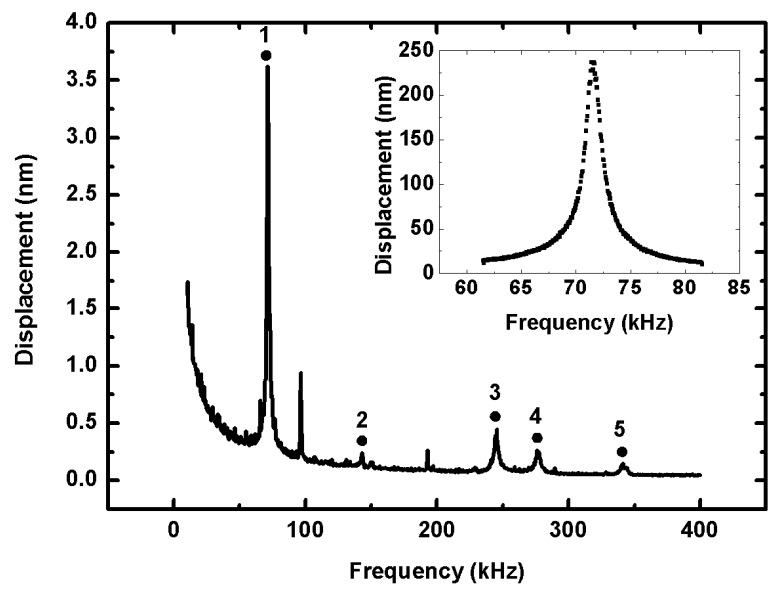

Figure 3.9. Frequency response of a $2000 \mu \mathrm{m}$ diameter membrane excited by a white noise signal. Resonant peaks are indicated by solid circles while numbers are the mode numbers. The peaks appearing at 100 and $200 \mathrm{kHz}$ are due to the measurement set-up. The inset shows the first resonant peak of the membrane excited by a sinusoidal voltage of $1 \mathrm{~V}$ amplitude.

The membrane is also actuated by a sinusoidal signal with the amplitude of $1 \mathrm{~V}$ in the range from 61 to $81 \mathrm{kHz}$, near its first resonant frequency. The resonant peak is obtained, as shown in the inset of figure 3.9. Using the same techniques, the first resonant peak on different membranes is 
found, as shown in figure 3.11. The resonant amplitude increases with the increase in the diameter of membranes. The best sensitivity of $0.24 \mu \mathrm{m} \mathrm{V}^{-1}$ is achieved on the $2000 \mu \mathrm{m}$ diameter membrane.

(a)

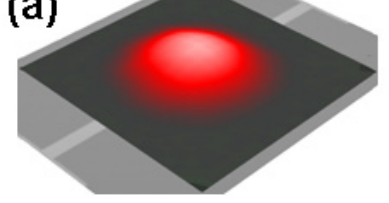

(b)
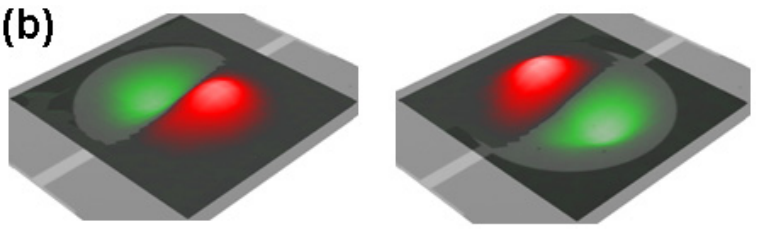

(c)
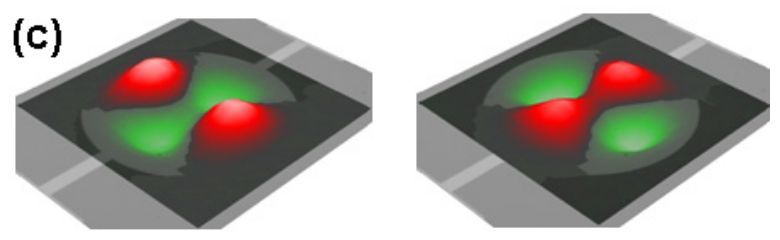

(d)
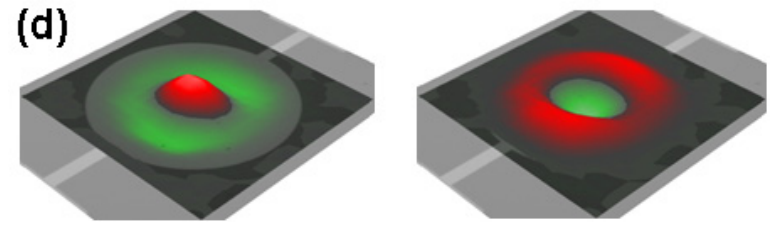

(e)
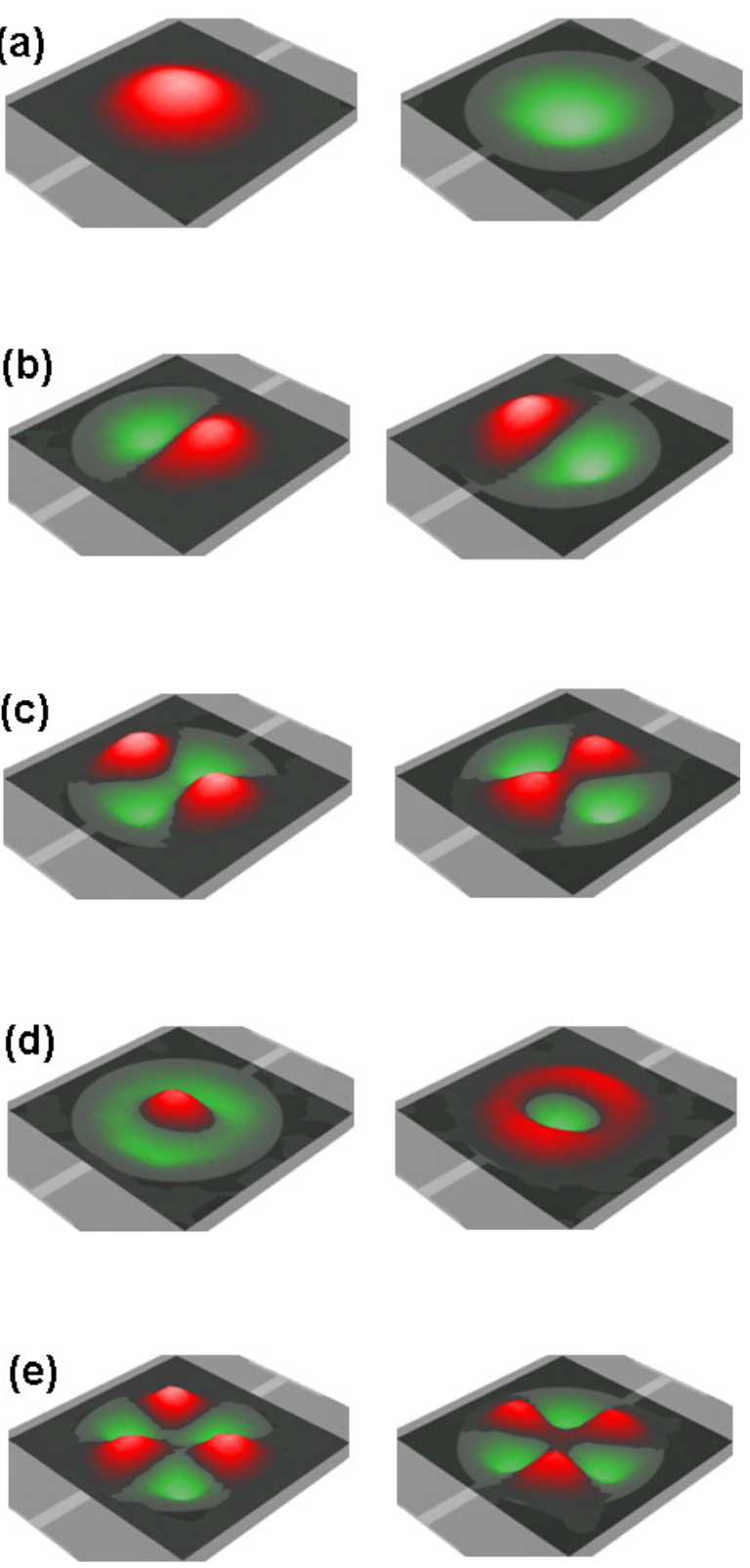

. 


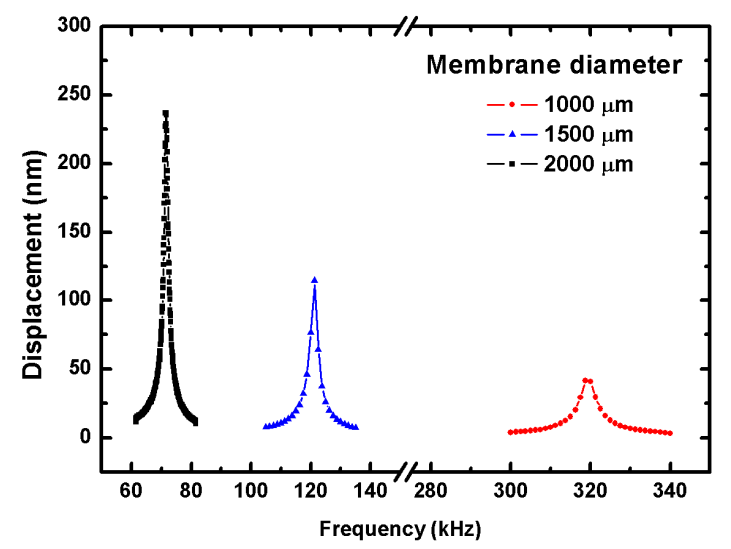

Figure 3.11. First resonant peaks of different size membranes.

By using the above techniques, the dynamic behavior of a $2500 \mu \mathrm{m}$ long cantilever is also investigated. Figure 3.12 shows the frequency spectrum of the cantilever excited by a white noise signal of $1 \mathrm{~V}$ with frequencies varying between 3 and $300 \mathrm{kHz}$. The first five modes of resonances are found to be at $8.13,50.63,96.56,192.88$, and $285.94 \mathrm{kHz}$. The insets of figure 3.12 illustrate the corresponding modal shapes obtained using the laser scanning. The cantilever was then excited at its first resonant frequency by applying $1 \mathrm{~V}$ sinusoidal signal. The sensitivity of $5 \mu \mathrm{m} \mathrm{V} \mathrm{V}^{-1}$ was achieved as shown in figure 3.13 .

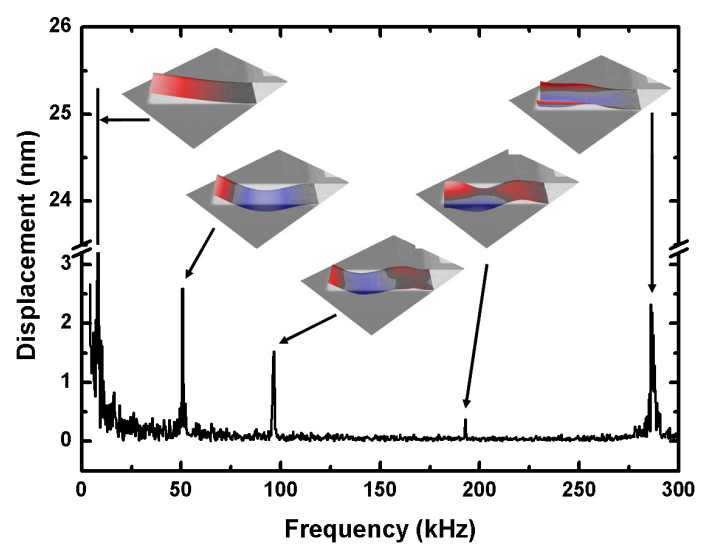

Figure 3.12. Typical frequency response of a cantilever $\left(1000 \times 2500 \times 40 \mu \mathrm{m}^{3}\right)$. The corresponding modal shapes are shown in the insets. 


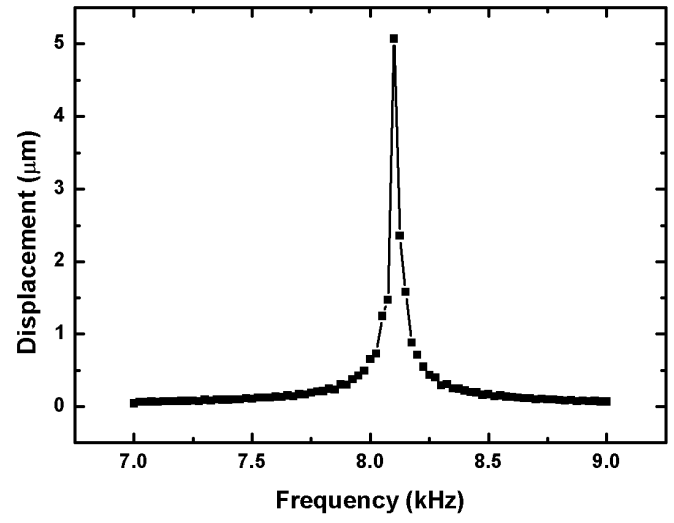

Figure 3.13. Tip displacement of the cantilever as a function of the frequency.

In resonant applications, the quality factor ( $Q$ factor) is another important parameter. For instance, the sensitivity of resonant devices used for detection of small forces depends on the spectral resolution, which is proportional to the $Q$ factor of the resonant mode. Therefore, a higher $Q$ factor is very much preferred. The $Q$ factor of our transducers was analyzed either in atmospheric pressure or in vacuum, for the fundamental mode of resonance. The $Q$ factor is defined as the ratio between the resonant frequency $\left(f_{r}\right)$ and the width of resonant peak at its half height $(\Delta f)$, i.e. $Q=f_{r} / \Delta f$. The transducers, placed in a vacuum chamber, are excited by applying a sinusoidal signal of $1 \mathrm{~V}$ (Hewlett Packard HP 33120A function generator), and the vibration velocity of the transducers was measured using the Polytec OFV 502 fiber interferometer incorporating with the OFV 3000 vibrometer controller. By using a DSO1014A oscilloscope (Agilent Technologies Inc.), the output voltage of the vibrometer is recorded. Such voltage is directly proportional to the deflection of transducers. The whole measurement system is set on a vibration isolation table to avoid mechanical noise.

Typical frequency spectra for a $1500 \mu \mathrm{m}$ diameter membrane and a $2500 \mu \mathrm{m}$ long cantilever under different pressure conditions are shown in figure 3.14. We observe that the resonant peaks increases and shifts to higher frequency with decreasing the pressure. This phenomenon is due to the reduction of air damping around the structures. In the case of the membrane, the $Q$ factor is 50 in atmospheric pressure, and increased markedly to 323 under the vacuum of 0.1 mbar. A sharp resonant peak is obtained. On the other hand, the resonant peak of the cantilever slightly increases under vacuum. It was argued that cantilever structures are less affected by the air damping compared to membrane structures [38]. In atmospheric pressure, our cantilever exhibited a high $Q$ factor of 169. Such a value, obtained for an epitaxial structure, is better to the one recently observed in sensors based on polycrystalline films [39]. Under a pressure of $0.1 \mathrm{mbar}$, the $Q$ factor is measured to be 284 . 

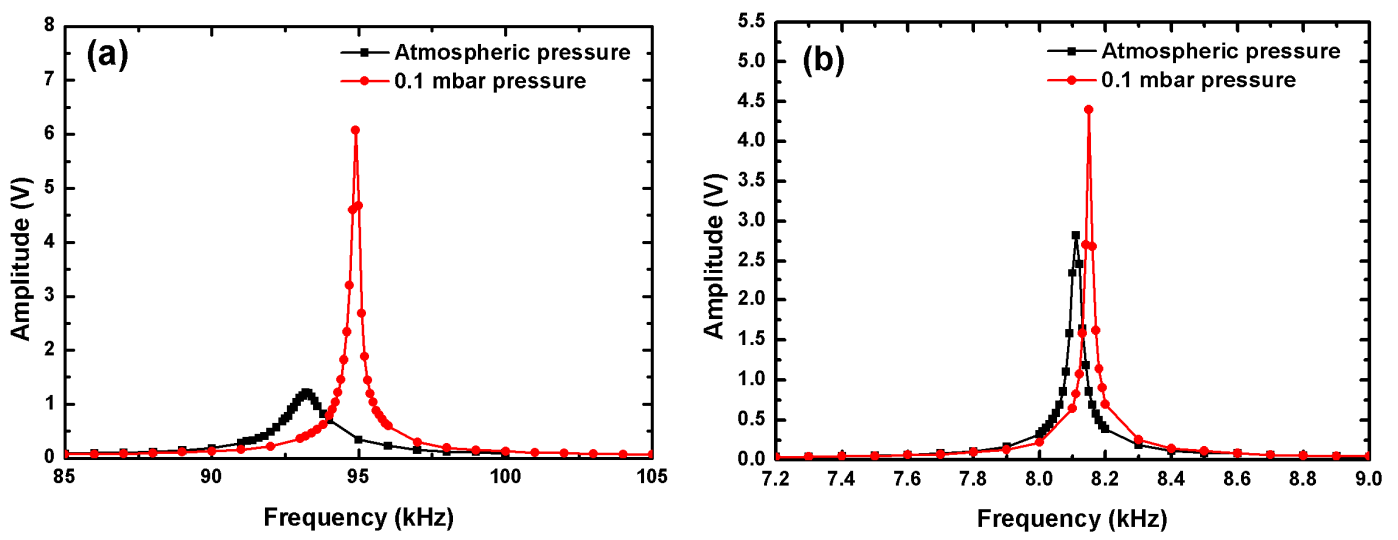

Figure 3.14. Frequency spectra for (a) a $1500 \mu \mathrm{m}$ diameter membrane and (b) a $2500 \mu \mathrm{m}$ long cantilever under atmospheric and 0.1 mbar pressures.

All experimental results presented in this section reveal the potentials of epitaxial piezoelectric MEMS devices both in static and dynamic working operations. An interesting aspect of the epitaxial PZT transducers is that the operational voltage is low compared to transducers actuated with polycrystalline PZT films. A large displacement can be obtained from the epitaxial PZT films due to excellent piezoelectric properties. Moreover, a strong harmonic oscillation with a high quality factor in dynamic mode can be achieved.

\subsection{Conclusions}

In conclusion, we have demonstrated the fabrication of piezoelectric MEMS based on epitaxial thin films prepared on silicon wafers. $\mathrm{Pb}\left(\mathrm{Zr}_{0.2} \mathrm{Ti}_{0.8}\right) \mathrm{O}_{3}$ thin films epitaxially grown on 2" (001) silicon substrates with an oxide bottom electrode exhibit high crystalline quality and good piezoelectric properties. The microfabrication process has been optimized for these oxide layers with special emphasis in maintaining the piezoelectric properties of the epi-piezo films. The measured polarization revealed no degradation of the PZT properties throughout the process. In order to analyze the behavior of the fabricated transducers, their static and dynamic characteristics have been investigated. A 100-nm thin epitaxial PZT film is able to flex the $2000 \mu \mathrm{m}$ diameter membrane by more than 25 $\mu \mathrm{m}$ at $4 \mathrm{~V}$, while a total displacement of $4 \mu \mathrm{m}$ is obtained for the $40 \mu \mathrm{m}$ thick cantilever. The epitaxial PZT transducers demonstrate relatively large deflections at low bias voltages in the static mode, which could be of great interest in many applications including micro pumps, micro valves, or deformable micro mirrors. In the dynamic mode, the epitaxial PZT cantilever exhibits a deflection of several microns. The membrane with a diameter of $1500 \mu \mathrm{m}$ resonating at $95 \mathrm{kHz}$ exhibits an excellent quality factor of 323 under vacuum, which is particularly suitable for the development of 
ultrasensitive mass sensors. The integration of epitaxial perovskite thin films on silicon is a promising technology, which could allow new advanced MEMS to be realized.

\section{Acknowledgements}

We acknowledge financial support by the Swiss National Science Foundation through the National Centre of Competence in Research 'Materials with Novel Electronic Properties' MaNEP and by the European Union through the project 'Nanoxide'. The work at Yale was supported by NSF MRSEC DMR 0520495 and DMR 0705799, along with support from SRC and the Packard and Sloan Foundations. We would like to thank Mr. Henri Haquette for precious assistance on the laser micromachining process and Prof. Herbert Shea for his advice in the dynamic measurements. Don Isarakorn is grateful to the Ministry of Science and Technology, Thailand for the financial support for his PhD studies at EPFL IMT-NE SAMLAB. 


\section{References}

[1] Wang Y, Ganpule C, Liu B T, Li K, Mori K, Hill B, Wuttig M, Ramesh R, Finder J, Yu Z, Droopad R and Eisenbeiser K 2002 Epitaxial ferroelectric $\mathrm{Pb}\left(\mathrm{Zr}\right.$, Ti) $\mathrm{O}_{3}$ thin films on Si using $\mathrm{SrTiO}_{3}$ template layers Appl. Phys. Lett. 80 97-99

[2] Chonge S G, Goo E, Ramesh R, Haakenaasen R and Fork D K 1994 Microstructure of epitaxial oxide thin film heterostructures on silicon by pulsed laser deposition Appl. Phys. Lett. 64 3407-09

[3] Maruyama T, Saitoh M, Sakai I, Hidaka T, Yano Y and Noguchi T 1998 Growth and characterizationof 10-nm-thick $c$-axis oriented epitaxial $\mathrm{PbZr}_{0.25} \mathrm{Ti}_{0.75} \mathrm{O}_{3}$ thin films on (100)Si substrate Appl. Phys. Lett. 73 3524-26

[4] Wang J, Zheng H, Ma Z, Prasertchoung S, Wuttig M, Droopad R, Yu J, Eisenbeiser K and Ramesh R 2004 Epitaxial $\mathrm{BiFeO}_{3}$ thin films on Si Appl. Phys. Lett. 85 2574-76

[5] Lin A, Hong X, Wood V, Verevkin A A, Ahn C H, McKee R A, Walker F J and Specht E D 2001 Epitaxial growth of $\mathrm{Pb}\left(\mathrm{Zr}_{0.2} \mathrm{Ti}_{0.8}\right) \mathrm{O}_{3}$ on $\mathrm{Si}$ and its nanoscale piezoelectric properties Appl. Phys. Lett. 78 2034-36

[6] Ramesh R and Schlom D G 2002 Orienting Ferroelectric Films Science 296 1975-1976

[7] Guerrero C, Ferrater C, Roldan J, Trtik V, Sanchez F and Varela M 2000 Epitaxial ferroelectric $\mathrm{PbZr}_{\mathrm{x}} \mathrm{Ti}_{1-\mathrm{x}} \mathrm{O}_{3}$ thin films for non-volatile memory applications Microelectron. Reliab. 40 671-74

[8] Reilly E K and Wright P K 2009 Modeling, fabrication and stress compensation of an epitaxial thin film piezoelectric microscale energy scavenging device J. Micromech. Microeng 19095014

[9] Biasotti M, Pellegrino L, Bellingeri E, Bernini C, Siri A S and Marre D 2009 All-oxide crystalline microelectromechanical systems Procedia Chemistry 1 839-42

[10] Okada N, Higuchi K, Kobayashi K, Ito M, Takabe M, Otonari M, Kanja I, Akai D, Sawada K and Ishida M 2008 Fabrication of MEMS diaphragm transducer array based on epitaxial PZT thin film for 2-D hydrophone application IEEE International Ultrasonics Symposium Proceedings, (Beijing, China) 1781-4

[11] Muralt P 2000 PZT thin films for microsensors and actuators: Where do we stand? IEEE Trans. Ultrason. Ferroelectr. Freq. Control 47 903-15

[12] Muralt P 2000 Ferroelectric thin films for micro-sensors and actuators: a review $J$. Micromech. Microeng 10 136-46

[13] Damjanovic D, Muralt P and Setter N 2001 Ferroelectric sensors IEEE Sens. J. 1 191-206

[14] Trolier-McKinstry S and Muralt P 2004 Thin film piezoelectrics for MEMS J. Electroceram. 12 7-17

[15] Baborowski J 2004 Microfabrication of piezoelectric MEMS Integr. Ferroelectr. 66 3-17 
[16] Muralt P 2008 Recent progress in materials issues for piezoelectric MEMS J. Am. Ceram. Soc. 91 1385-96

[17] McKee R A, Walker F J and Chisholm M F 1998 Crystalline oxides on silicon: The first five monolayers Phys. Rev. Lett. 81 3014-17

[18] Reiner J W, Garrity K F, Walker F J, Ismail-Beigi S and Ahn C H 2008 Role of strontium in oxide epitaxy on silicon (001) Phys. Rev. Lett. 101105503

[19] Gariglio S, Stucki N, Triscone J -M and Triscone G 2007 Strain relaxation and critical temperature in epitaxial ferroelectric $\mathrm{Pb}\left(\mathrm{Zr}_{0.20} \mathrm{Ti}_{0.80}\right) \mathrm{O}_{3}$ thin films Appl. Phys. Lett. 90202905

[20] See, for example, Tybell T, Ahn C H and Triscone J -M 1998 Control and imaging of ferroelectric domains over large areas with nanometer resolution in atomically smooth epitaxial $\mathrm{Pb}\left(\mathrm{Zr}_{0.2} \mathrm{Ti}_{0.8}\right) \mathrm{O}_{3}$ thin films Appl. Phys. Lett. 72 1454-56 and references therein.

[21] Shimamoto $\mathrm{Y}$, Kushida-Abdelghafar K, Miki H and Fujisaki $\mathrm{Y} 1997 \mathrm{H}_{2}$ damage of ferroelectric $\mathrm{Pb}(\mathrm{Zr}, \mathrm{Ti}) \mathrm{O}_{3}$ thin-film capacitors-The role of catalytic and adsorptive activity of the top electrode Appl. Phys. Lett., 70 3096-97

[22] Paruch P and Triscone J -M 2006 High-temperature ferroelectric domain stability in epitaxial $\mathrm{PbZr}_{0.2} \mathrm{Ti}_{0.8} \mathrm{O}_{3}$ thin films Appl. Phys. Lett. 88162907

[23] Isarakorn D, Briand D, Gariglio S, Sambri A, Stucki N, Triscone J -M, Guy F, Baek S -H, Eom C -B, Reiner J W, Ahn C H and de Rooij N F 2008 Establishment of a technology platform for epitaxial piezoelectric MEMS Proceedings of EUROSENSORS 2008, (Dresden, Germany) 819-22

[24] Peng J, Chao C, Dai J, Chan H L W and Lua H 2008 Micro-patterning of $0.70 \mathrm{~Pb}\left(\mathrm{Mg}_{1 / 3} \mathrm{Nb}_{2 / 3}\right) \mathrm{O}_{3}-0.30 \mathrm{PbTiO}_{3}$ single crystals by ultrasonic wet chemical etching Mater. Lett. 62 3127-30

[25] Zeto R J, Rod B J, Dubey M, Ervin M H, Piekarz R C, Trolier-McKinstry S, Su T and Shepard J F 1998 High-resolution dry etch patterning of PZT for piezoelectric MEMS devices Proceedings of the Eleventh IEEE International Symposium on Applications of Ferroelectrics, (Montreux, Switzerland) 89-92

[26] Giesbers J B, Prins M W J, Cillessen J F M and van Esch H A 1997 Dry etching of all-oxide transparent thin film memory transistors Microelectron. Eng. 35 71-4

[27] Nagaraj B, Aggarwal S and Ramesh R 2001 Influence of contact electrodes on leakage characteristics in ferroelectric thin films J. Appl. Phys. 90, 375-82

[28] Allard M, Boughaba S and Meunier M 1997 Laser micromachining of free-standing structures in $\mathrm{SiO}_{2}$-covered silicon Appl. Surf. Sci. 109-110, 189-93

[29] Most D, Choi J, Belenky L J and Eom C B 2003 Laser micromachining of $\mathrm{SrTiO}_{3}$ single crystal Solid-State Electron. 47 2249-53

[30] Zhang B and Yung K C 2006 Frequency-tripled Nd:YAG laser ablation in laser structuring process Opt. Lasers Eng. 44 815-25 
[31] Hong E, Trolier-McKinstry S, Smith R L, Krishnaswamy S V and Freidhoff C B 2006 Design of MEMS PZT circular diaphragm actuators to generate large deflections $J$. of Microelectromech. Syst. 15 832-39

[32] Hishinuma Y, Yang E -H, Cheng J -G and Trolier-McKinstry S 2004 Optimized design, fabrication and characterization of PZT unimorph microactuators for deformable mirrors ASME 2004 International Mechanical Engineering Congress and Exposition, (California, USA) 299-304

[33] Smits J and Choi W 1991 The constituent equations of piezoelectric heterogeneous bimorphs IEEE Trans. Ultrason. Ferroelectr. Freq. Control 38 256-70

[34] Luginbuhl Ph, Racine G -A, Lerch Ph, Romanowicz B, Brooks K G, de Rooij N F, Renaud Ph and Setter N 1996 Piezoelectric cantilever beams actuated by PZT sol-gel thin Sens. Actuators, A 54 530-5

[35] 1990 Landolt-Börnstein: Numerical Data and Functional Relationships in Science and Technology New serie, Group III, vol 26 (Berlin: Springer-Verlag) p 123

[36] Hoffmann M, Kuppers H, Schneller T, Bottger U, Schnakenberg U, Mokwa W and Waser R 2003 Theoretical calculations and performance results of a PZT thin film actuator IEEE Trans. Ultrason. Ferroelectr. Freq. Control 50 1240-46

[37] Haccart T, Cattan E and Remiens D 2002 Dielectric, ferroelectric and piezoelectric properties of sputtered PZT thin films on Si substrates: influence of film thickness and orientation Semicond. Phys. - Quantum Electronics and Optoelectronics 77 78-88

[38] Hong E, Trolier-McKinstry S, Smith R, Krishnaswamy S V and Freidhoff C B 2006 Vibration of micromachined circular piezoelectric diaphragms IEEE Trans. Ultrason. Ferroelectr. Freq. Control 53 697-706

[39] Kwon T Y, Eom K, Park J H, Yoon D S, Kim T S and Lee H L 2007 In situ real-time monitoring of biomolecular interactions based on resonating microcantilevers immersed in a viscous fluid Appl. Phys. Lett. 90223903 


\title{
Chapter 4
}

\section{Electrical characteristics of piezoelectric energy harvesters based on an epitaxial $\mathrm{Pb}\left(\mathrm{Zr}_{0.2} \mathbf{T i}_{0.8}\right) \mathrm{O}_{3}$ thin film}

\author{
D. Isarakorn ${ }^{1}$, D. Briand ${ }^{1}$, P. Janphuang ${ }^{1}$, N. F. de Rooij ${ }^{1}$, J. W. Reiner ${ }^{2}$ and C. H. Ahn ${ }^{2}$ \\ ${ }^{1}$ École Polytechnique Fédérale de Lausanne (EPFL), Institute of Microengineering (IMT), \\ The Sensors, Actuators and Microsystems Laboratory (SAMLAB), \\ Rue Jaquet-Droz 1, P.O. Box 526, 2002 Neuchâtel, Switzerland \\ ${ }^{2}$ Department of Applied Physics, Yale University, \\ Becton Center, P.O. Box 208284 New Haven, CT 06520-8284 USA
}

\begin{abstract}
This letter investigates the electrical characteristics of a piezoelectric cantilever for vibration energy harvesting by utilizing an epitaxial $\mathrm{Pb}\left(\mathrm{Zr}_{0.2} \mathrm{Ti}_{0.8}\right) \mathrm{O}_{3}$ thin film. The electrical characteristics of the epitaxial piezoelectric harvester are analyzed in the viewpoint of material properties and compared with other piezoelectric harvesters. The outcome reveals that the piezoelectric harvester realized with epitaxial piezoelectric film enables large power generation, low optimal resistive load, and high output current. The power generation of the epitaxial device $\left(0.1325 \mathrm{~mm}^{3}\right)$ measured at its resonant frequency $(2.3 \mathrm{kHz})$ produces $14 \mu \mathrm{W} g^{-2}, 60 \mu \mathrm{A} \mathrm{g}^{-1}$ and $0.28 \mathrm{~V} \mathrm{~g}^{-1}$ for an optimal resistive load of 4.7 $\mathrm{k} \Omega$.
\end{abstract}




\subsection{Introduction}

MEMS based energy harvesters are attractive as an alternative power source for low-power electronic devices and sensors, with as one of their potential applications, their implementation in wireless sensor nodes. The development of harvesters to generate energy from vibrations has advanced rapidly during the past few years. Several transduction methods including electromagnetic induction, electrostatic generation, and piezoelectric materials have been discussed in review articles [1, 2]. Among these methods, piezoelectric materials have received the most attention due to directly convert vibration energy into electrical energy with a high power density and ease of integration into a system [3]. Several piezoelectric silicon micromachined energy scavengers, mainly based on polycrystalline lead zirconate titanate (PZT) and aluminum nitride (AIN) films, have been proposed by various research groups. Recently, a $d_{31}$-mode (transverse) piezoelectric harvester based on AlN films has been reported, which exhibits an excellent output power of $60 \mu \mathrm{W}$ [4]. The use of the $d_{33}$-mode (longitudinal) [5,6] has been demonstrated with the use of PZT cantilevers with the advantage of a higher generated voltage due to higher piezoelectric coefficient and ability to optimize capacitance by changing the top electrode patterns. For these different types of harvesters, many designs utilizing polycrystalline films have shown to generate interesting power levels requiring a high optimal resistive load. The power is of course a very important parameter, but their output current or voltage is not always optimum depending on the type of piezoelectric films and operating modes used. Current, voltage and load are of considerable importance and are function of the properties of the piezoelectric film used, the later being therefore a key issue to consider to improve the overall performance of energy harvesters

We have demonstrated that the properties of epitaxial piezoelectric films lead to MEMS devices with superior performances [7], and we are now evaluating the benefits that these epitaxial films could bring to mechanical energy harvesters. So far, the vibration energy harvesting involving epitaxial PZT (epi-PZT) thin films have been seldom reported [8, 9]. In this letter, we investigate the use of an epitaxial $\mathrm{Pb}\left(\mathrm{Zr}_{0.2} \mathrm{Ti}_{0.8}\right) \mathrm{O}_{3}$ film and its electrical properties for energy harvesting applications. A very interesting outcome is that the epi-PZT harvester generates higher power and current with usable voltage, and requires lower optimal resistive load compared to piezoelectric harvesters realized with polycrystalline PZT (poly-PZT) or AlN films. We are also proposing an analysis of the results obtained in relation with the electrical characteristics of the different piezoelectric materials.

\subsection{Device fabrication and characteristics}

Our energy harvesting device consists of multilayered epitaxial oxide films deposited on a silicon cantilever beam ended with a Si proof mass designed based on our modeling [9]. $\mathrm{A} \mathrm{Pb}\left(\mathrm{Zr}_{0.2} \mathrm{Ti}_{0.8}\right) \mathrm{O}_{3}$ layer is grown by off-axis magnetron sputtering on a silicon substrate [7] through two oxide layers: a structural buffer $\mathrm{SrTiO}_{3}$ (STO) grown by MBE [10] and a bottom electrode $\mathrm{SrRuO}_{3}$ (SRO) grown by 
off-axis magnetron sputtering. The typical stack results as PZT $(500 \mathrm{~nm}) / \mathrm{SRO}(30 \mathrm{~nm}) / \mathrm{STO}(6$ $\mathrm{nm}) / \mathrm{Si}$. The residual stress present in the epitaxial films after growth was evaluated as low as $60 \mathrm{MPa}$ using a thin film stress measurement system (Tencor FLX-2320A). The harvester (figure 4.1) is then fabricated using micro-patterning techniques optimized for the oxide layers deposited on silicon as detailed in [7].

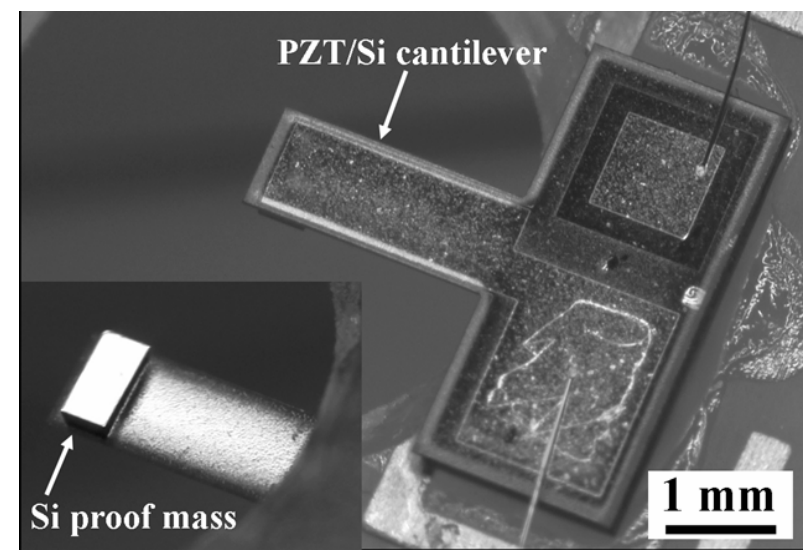

Figure 4.1. Optical image of an epi-PZT cantilever $\left(1000 \times 2500 \times 7 \mu \mathrm{m}^{3}\right)$ with a Si proof mass $\left(1000 \times 500 \times 230 \mu^{3}\right)$. The inset shows the Si proof mass on the back side of the cantilever. The effective volume of the final device is $0.1325 \mathrm{~mm}^{3}$.

The dielectric constant $\varepsilon_{r}$ of the epi-PZT thin films characterized by the measurement of the capacitance values on the $\mathrm{Cr} / \mathrm{Au}$ top electrodes of $100 \times 100 \mu \mathrm{m}^{2}$ area yields a value of $70-100$. The piezoelectric coefficient $d_{31}$ of the epi-PZT thin films was estimated from the displacement at the free end of the epi-PZT cantilevers $\left(1000 \times 2500 \times 40 \mu^{3}\right)$ using an interferometric profiler (Wyko NT110) [7]. We did not have to perform a poling treatment on our films prior to the measurements since they were naturally polarized. The deflections of the cantilevers coated with $100 \mathrm{~nm}$ thick epi-PZT films were measured as a function of dc voltage (figure 4.2(a)). Calculation of the piezoelectric coefficient $d_{31}$ yields a value of $135.4 \pm 7.1 \mathrm{pm} \mathrm{V}^{-1}$. Figure $4.2(\mathrm{~b})$ shows the $e_{31, f}$ as a function of applied voltage calculated following the derivation of Muralt [11]. The effective piezoelectric coefficient $e_{31, f}$ of $18.2 \pm 0.9 \mathrm{C} \mathrm{m}^{-2}$ is estimated for the epi-PZT (20/80) thin films, which is significantly higher than what has been reported in the past [12]. Such a high value of the effective piezoelectric coefficient $e_{31, f}$ could arise from $90^{\circ}$ domain-wall contributions due to partially released clamping of the cantilever structure, which gives rise to a substantial increase in the effective piezoelectric coefficients [13]. It should be noted that all $\varepsilon_{r}$ and $e_{31, f}$ values were obtained from different epi-PZT 
cantilevers located in the center area of the wafer, where the epi-PZT film have homogeneous thickness and quality.

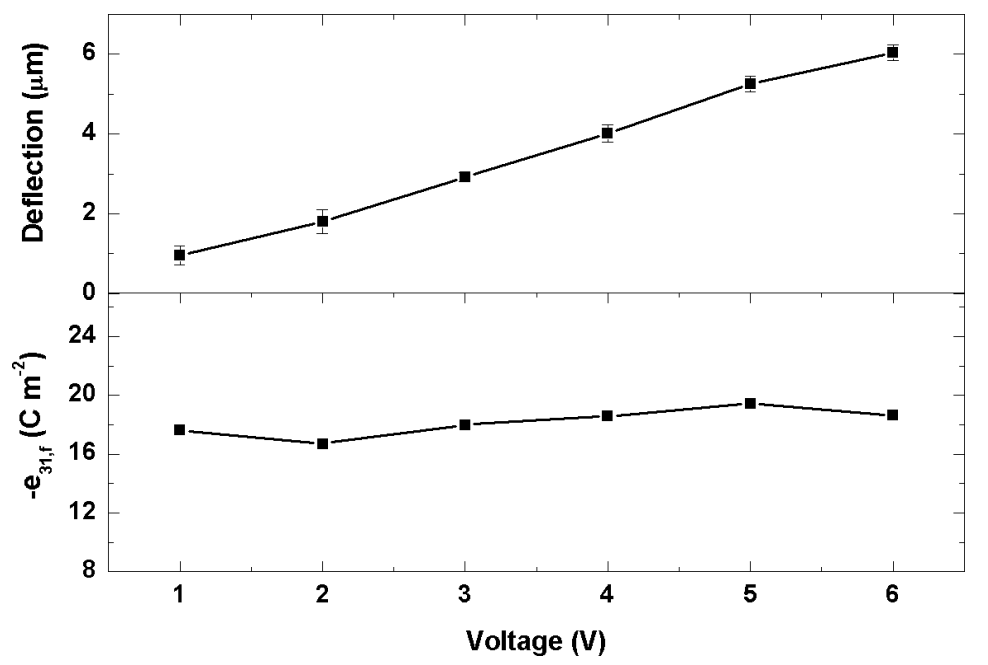

Figure 4.2. (a) Cantilever deflection and (b) effective piezoelectric coefficient under dc excitation. The effective piezoelectric coefficient was calculated from the measured $d_{31}$ and the elastic constants $s_{11}^{E}\left(10.8 \times 10^{-12} \mathrm{~m}^{2} \mathrm{~N}^{-1}\right)$ and $s_{12}^{E}\left(-3.35 \times 10^{-12} \mathrm{~m}^{2} \mathrm{~N}^{-1}\right)$ from [14].

In PZT thin films, a variety of factors, including orientation, grain size, strain effect, and composition all impacts the piezoelectric properties. Poly-PZT thin films appear often to follow bulk behavior; their properties are strongly affected by the $\mathrm{Zr} / \mathrm{Ti}$ ratio. Their piezoelectric, dielectric, and ferroelectric properties are maxima near the morphotropic phase boundary $[15,16]$. However, it has been reported that the behaviors of epitaxial or highly oriented films were quite different from those of bulk ceramics $[15,17]$. Therefore, the high piezoelectric coefficient obtained from our epi-PZT (20/80) films is a primary consequence of the high degree of crystallographic orientation in the epitaxial films as well as the composition of the epi-PZT having a low lattice mismatch with the substrate. The latter is considered to be a key parameter to reduce strain effects, and thus further improve the piezoelectric performance [18, 19]. Moreover, owing to decreased substrate clamping, the movement of $90^{\circ}$ domain walls in epi-PZT cantilevers increases the effective piezoelectric coefficient, which is larger than the theoretical value of a bulk single crystal. The microfabrication process optimized for these oxide layers is also a crucial factor to maintain the excellent piezoelectric properties of the epi-PZT films. As the result, the epi-PZT (20/80) films exhibit a high piezoelectric coefficient and a low dielectric permittivity resulting in a high electromechanical coupling factor, which can enhance the performance of piezoelectric energy harvesters [20]. 


\subsection{Results and discussion}

In the experiment, the resonant frequency $f_{r}$ of the epi-PZT cantilever is first characterized using a Polytec MVS-400 laser Doppler vibrometer, and found at $2302 \mathrm{~Hz}$. The power generation performance of the device is then investigated with a shaker (Bruel \& Kjaer type 4811) driven by a vibration exciter control (type 1050) and a power amplifier (type 2712), by applying an acceleration as a mechanical input. The device is connected with various resistive loads $R_{L}$, and the current $I_{\text {rms }}$ generating through a resistive load under different acceleration levels is recorded with a multimeter (Agilent 34411A). The corresponding average power is calculated by $P_{\text {ave }}=I_{\mathrm{rms}}^{2} R_{L}$. The output current, average power and voltage $V_{\text {rms }}$ as a function of resistive loads are shown in figure 4.3. These output data are normalized per square acceleration $\left(g^{2}\right)$ for output power and per acceleration $(g)$ for current and voltage in order to compare its performance with other MEMS energy harvesters. A maximum output power of $14 \mu \mathrm{W} \mathrm{g} g^{-2}$ with $60 \mu \mathrm{A} \mathrm{g}^{-1}$ output current from a single device is obtained for an optimal resistive load of $4.7 \mathrm{k} \Omega$, and thus resulting in the output voltage of $0.28 \mathrm{~V} \mathrm{~g}^{-1}$. The maximum power density is as high as $105.66 \mu \mathrm{W} \mathrm{g}^{-2} \mathrm{~mm}^{-3}$.
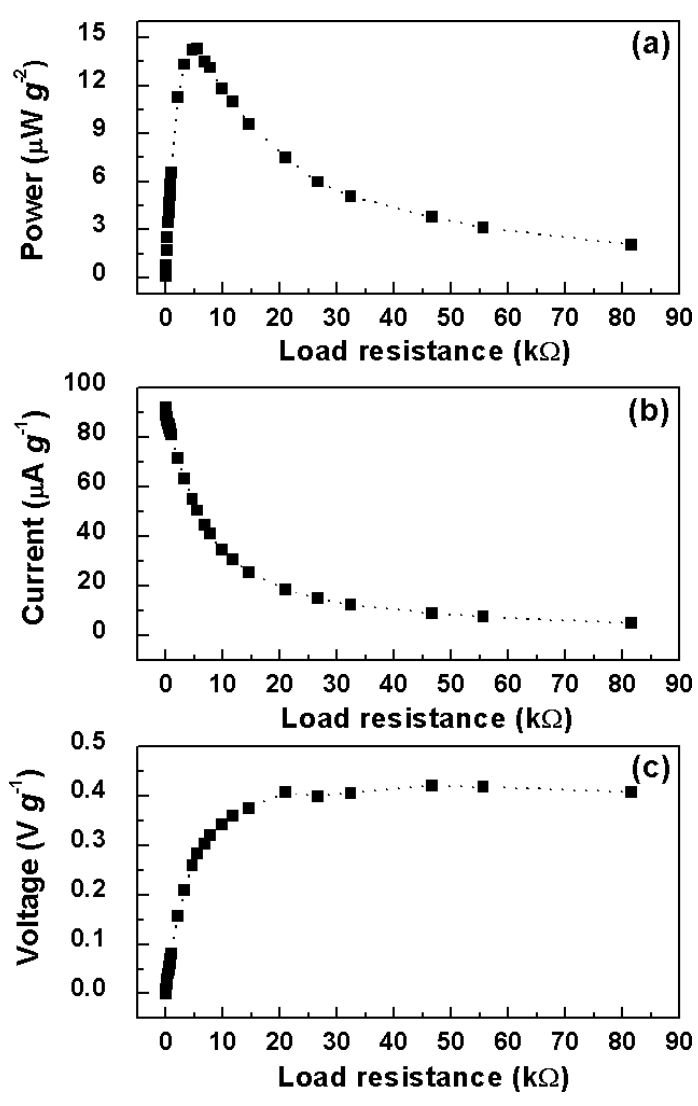

Figure 4.3. (a) Average power, (b) output current and (c) corresponding output voltage of an epi-PZT harvester versus resistive load. 
Table 4.1 shows the electrical output characteristics of the energy harvester based on the epiPZT film compared with other piezoelectric harvesting devices based on poly-PZT and AlN films. The epi-PZT harvester produces superior performances, higher current and smaller resistive load, due to excellent ferroelectric properties. Although, the power generated by the epi-PZT is similar to that of AIN, the epi-PZT harvester exhibits the highest power density, which is of high interest when realizing miniaturized devices. Usually, energy harvesting devices with a low optimal load resistance are highly desirable because they can generate high output current, and also their impedance can be easily matched to standard electronic devices. Referring to the table, the energy harvesting devices based on poly-PZT and AlN films demonstrated useful power generation, but their high impedances limit the output current. Considering the poly-PZT films with different operation modes, the power generated from the $d_{31}$-mode devices is mainly due to higher current than voltage, while the $d_{33}$ mode devices can generate higher voltage at the cost of producing a lower current. Unlike the polycrystalline film based devices, the device reported here using a thin epi-PZT film can generate high power and current with usable voltage, while maintaining low optimal resistive load.

Table 4.1. Performance comparison among MEMS energy harvesters.

\begin{tabular}{|c|c|c|c|c|c|c|c|c|c|}
\hline Reference & Type of device & $\begin{array}{c}\text { Piezo thickness } \\
(\mu \mathrm{m})\end{array}$ & $\begin{array}{c}\text { Effective volume } \\
\left(\mathrm{mm}^{3}\right)\end{array}$ & $\begin{array}{c}\text { Power } \\
\left(\mu W g^{-2}\right)\end{array}$ & $\begin{array}{l}\text { Power density } \\
\left(\mu \mathrm{W} g^{-2} \mathrm{~mm}^{-3}\right)\end{array}$ & $\begin{array}{c}\text { Voltage density } \\
\left(\mathrm{V}^{-1} \mathrm{~mm}^{-3}\right)\end{array}$ & $\begin{array}{c}\text { Current density } \\
\left(\mu \mathrm{A} g^{-1} \mathrm{~mm}^{-3}\right)\end{array}$ & $\begin{array}{c}\text { Frequency } \\
(\mathrm{Hz})\end{array}$ & $\begin{array}{c}\text { Optimal load } \\
(\mathrm{k} \Omega)\end{array}$ \\
\hline Fang et al [21] & $d_{31}$ PZT & 1.64 & 0.1944 & 2.16 & 11.11 & 1.06 & 49.5 & 608 & 21.4 \\
\hline Shen et al [22] & $d_{31}$ PZT & 1 & 0.6520 & 0.54 & 0.82 & 0.09 & 14.57 & 462.5 & 6 \\
\hline Jeon et al [5] & $d_{33}$ PZT & 0.48 & 0.0270 & 0.01 & 0.32 & 7.85 & 1.52 & 13900 & 5200 \\
\hline Lee et al [6] & $d_{33}$ PZT & 5 & 0.6120 & 0.32 & 0.53 & 1.19 & 2.34 & 214 & 510 \\
\hline Elfrink et al [4] & $d_{31} \mathrm{AlN}$ & 0.8 & $>12.7272$ & 15 & $<1.18$ & $<0.20$ & $<0.45$ & 572 & $100-1000$ \\
\hline This research & $d_{31}$ epitaxial PZT & 0.5 & 0.1325 & 14 & 105.66 & 2.11 & 449.62 & 2302 & 4.7 \\
\hline
\end{tabular}

Based on the piezoelectric properties of the different films, we will in the following compare the electrical characteristics of the piezoelectric harvesters made of different films, identifying which properties make the epi-PZT exhibiting a unique behavior. The figures of merit for the power $\left(\mathrm{P}_{\mathrm{F}}\right)$ : $e_{31, f}^{2} / \varepsilon_{r}$, the voltage $\left(\mathrm{V}_{\mathrm{F}}\right): e_{31, f} / \varepsilon_{r}$, the current $\left(\mathrm{I}_{\mathrm{F}}\right): e_{31, f}$, and the electromechanical coupling $\left(\mathrm{K}_{\mathrm{F}}\right)$ : $e_{31, f}^{2} / \varepsilon_{r}$ were used for comparison purpose [12]. From reference [12], the $e_{31, f}$ and $\varepsilon_{r}$ values for the AlN are $-1.05 \mathrm{C} \mathrm{m}^{-2}$ and 10.5 respectively, while for the poly-PZT $(\mathrm{Zr} / \mathrm{Ti}=53 / 47)$ these values are $12 \mathrm{C} \mathrm{m}^{-2}$ for $e_{31, f}$ and 900 for $\varepsilon_{r}$. Thus, the $\mathrm{P}_{\mathrm{F}}$ values of the AlN (0.11) and the poly-PZT (0.16) are much lower than that of epi-PZT (3.31). The epi-PZT based harvester can generate more power than the other piezoelectric films because of its higher $\mathrm{P}_{\mathrm{F}}$. The higher current output obtained from the epiPZT can be explained by its superior piezoelectric coefficient. The $\mathrm{V}_{\mathrm{F}}$ value of AlN is similar to that of epi-PZT, while the poly-PZT exhibits the lowest $\mathrm{V}_{\mathrm{F}}$. Concerning the resistive load, the optimal resistive load $R_{L}$ can be determined through the non-dimensional parameter $\psi=R_{L} C\left(2 \pi f_{r}\right)$, where $C$ is the harvester capacitance [23]. This parameter depends strongly on an electromechanical 
coupling coefficient $k$, where $\psi=1$ for a low $k$ value and $\psi \ll 1$ for a high $k$ value. The poly-PZT and AlN devices are considered as a low coupling system (low $\mathrm{K}_{\mathrm{F}}$ value), their optimal resistive loads can be calculated at $\psi=1$ by $R_{L}=1 /\left(2 \pi f_{r} C\right)$. Therefore, the AlN devices require a much higher optimal resistive load than the poly-PZT devices due to a lower dielectric constant. Considering harvesters based on the epi- and poly-PZT thin films, the poly-PZT has a higher capacitance value than the epi-PZT. However, the coupling of the epi-PZT devices is much higher due to a high $\mathrm{K}_{\mathrm{F}}$ value $(\psi \ll 1)$. Consequently, the epi-PZT harvesters require a lower optimal load, which is favorable for the impedance matching with electronic devices.

\subsection{Conclusions}

In summary, the electrical characteristics of a vibration energy harvester based on an epitaxial $\mathrm{Pb}\left(\mathrm{Zr}_{0.2} \mathrm{Ti}_{0.8}\right) \mathrm{O}_{3}$ thin film have been evaluated analytically and experimentally in comparison with other piezoelectric films. The measurements demonstrated that the epi-PZT thin films provided a large piezoelectric coefficient $e_{31, f}$ of $18.2 \mathrm{C} \mathrm{m}^{-2}$ without a poling treatment and a low dielectric constant of 100 , which are key parameters to realize high performance piezoelectric energy harvesters. The epi-PZT harvester can achieve a maximum power density of $105.66 \mu \mathrm{W} \mathrm{g} \mathrm{gm}^{-2}$ at a low optimal load of $4.7 \mathrm{k} \Omega$. Based on this study, the epi-PZT film has a high potential for vibration energy harvesting applications as it can provide high electrical output at a low optimal load. Future work will address on the influence of epi-PZT composition, crystal structure and thickness as well as device optimization.

\section{Acknowledgements}

We gratefully acknowledge A. Sambri, S. Gariglio, and Prof. J -M Triscone (Dept. of Condensed Matter Physics, University of Geneva) for help with thin film preparation and helpful discussions. This work was supported by the Swiss National Science Foundation through the National Centre of Competence in Research 'Materials with Novel Electronic Properties' MaNEP and by the European Union through the project 'Nanoxide'. The work at Yale was supported by NSF MRSEC DMR 0520495 and the FENA center, along with support from SRC and the Packard and Sloan Foundations. 


\section{References}

[1] Beeby S P, Tudor M J and White N M 2006 Energy harvesting vibration sources for Microsystems applications Meas. Sci. Technol. 17 R175-R195

[2] Mitcheson P D, Reilly E K, Toh T, Wright P K and Yeatman E M 2007 Performance limits of the three MEMS inertial energy generator transduction types J. Micromech. Microeng. 17 S211-S216

[3] Muralt P, Polcawich R G and Trolier-McKinstry S 2009 Piezoelectric thin films for sensors, actuators, and energy harvesting MRS Bull. 35 658-64

[4] Elfrink R, Kamel T M, Goedbloed M, Matova S, Hohlfeld D, van Andel Y and van Schaijk R 2009 Vibration energy harvesting with aluminum nitride-based piezoelectric devices $J$. Micromech. Microeng. 19094005

[5] Jeon Y B, Sood R, Jeong J -H and Kim S -G 2005 MEMS power generator with transverse mode thin film PZT Sens. Actuators, A 122 16-22

[6] Lee B S, Lin S C, Wu W J, Wang X Y, Chang P Z and Lee C K 2009 Piezoelectric MEMS generators fabricated with an aerosol deposition PZT thin film J. Micromech. Microeng. 19 065014

[7] Isarakorn D, Sambri A, Janphuang P, Briand D, Gariglio S, Triscone J -M, Guy F, Reiner J W, Ahn C H, de Rooij N F 2010 Epitaxial piezoelectric MEMS on silicon J. Micromech. Microeng. 20055008

[8] Reilly E K and Wright P K 2009 Modeling, fabrication and stress compensation of an epitaxial thin film piezoelectric microscale energy scavenging device J. Micromech. Microeng. 19095014

[9] Isarakorn D, Briand D, Gariglio S, Sambri A, Stucki N, Triscone J -M, Guy F, Reiner J W, Ahn C H and de Rooij N F 2008 A study on epitaxial piezoelectric thin films grown on silicon for energy scavenging applications Proc. PowerMEMS 2008 + MicroEMS 2008, (Sendai, Japan) pp. 269-72

[10] Su D, Yang B, Jiang N, Sawicki M, Broadbridge C, Couillard M, Reiner J W, Walker F J, Ahn C H and Zhu Y 2010 Valence electron energy-loss spectroscopy of ultrathin $\mathrm{SrTiO}_{3}$ films grown on silicon (100) single crystal Appl. Phys. Letter. 96121914

[11] Muralt P 1997 Piezoelectric thin films for MEMS Integr. Ferroelectr. 17 297-307

[12] Trolier-McKinstry S and Muralt P 2004 Thin film piezoelectric for MEMS J. Electroceram. 12 7-17 and references therein

[13] Ma Z, Zavaliche F, Chen L, Ouyang J, Melngailis J, Roytburd A L, Vaithyanathan V, Schlom D G, Zhao T and Ramesh R 2005 Effect of $90^{\circ}$ domain movement on the piezoelectric response of patterned $\mathrm{PbZr}_{0.2} \mathrm{Ti}_{0.8} \mathrm{O}_{3} / \mathrm{SrTiO}_{3} / \mathrm{Si}$ heterostructures Appl. Phys. Lett. 87072907 
[14] 1990 Landolt-Börnstein: Numerical Data and Functional Relationships in Science and Technology New serie, Group III, vol 26 (Berlin: Springer-Verlag) p 123

[15] Kim D -J, Maria J -P, Kingon A I and Streiffer S K 2003 Evaluation of intrinsic and extrinsic contributions to the piezoelectric properties of $\mathrm{Pb}\left(\mathrm{Zr}_{\mathrm{x}} \mathrm{Ti}_{1-\mathrm{x}}\right) \mathrm{O}_{3}$ thin films as a function of composition J. Appl. Phys. 93 5568-75

[16] Chen H D, Udayakumar K R, Gaskey C J and Cross L E 1995 Electrical properties' maxima in thin films of the lead zirconate-lead titanate solid solution system Appl. Phys. Lett. 673411

[17] Kanno I, Kotera H, Wasa K, Matsunaga T, Kamada T and Takayama R 2003 Crystallographic characterization of epitaxial $\mathrm{Pb}(\mathrm{Zr}, \mathrm{Ti}) \mathrm{O}_{3}$ films with different $\mathrm{Zr} / \mathrm{Ti}$ ratio grown by radio-frequency-magnetron sputtering J. Appl. Phys. 93 4091-6

[18] Kuffer O, Maggio-Aprile I, Triscone J -M, Fischer O and Renner Ch 2000 Piezoelectric response of epitaxial $\mathrm{Pb}\left(\mathrm{Zr}_{0.20} \mathrm{Ti}_{0.80}\right) \mathrm{O}_{3}$ films measured by scanning tunneling microscopy Appl. Phys. Lett. 77 1701-3

[19] Wang Y, Ganpule, Liu B T, Li H, Mori K, Hill B, Wuttig M, Ramesh R, Finder J, Yu Z, Droopad R and Eisenbeiser K 2002 Epitaxial ferroelectric $\mathrm{Pb}(\mathrm{Zr}, \mathrm{Ti}) \mathrm{O}_{3}$ thin films on Si using $\mathrm{SrTiO}_{3}$ template layers Appl. Phys. Lett. 80 97-9

[20] Morimoto K, Kanno I, Wasa Kiyotaka and Kotera H 2010 High-efficiency piezoelectric energy harvesters of $c$-axis-oriented epitaxial PZT films transferred onto stainless steel cantilevers Sens. Actuators, A 163 428-32

[21] Fang H -B, Liu J -Q, Xu Z -Y, Dong L, Wang L, Chen D, Cai B -C and Liu Y 2006 Fabrication and performance of MEMS-based piezoelectric power generator for vibration energy harvesting Microelectron. J. 37 1280-4

[22] Shen D, Park J -H, Ajitsaria J, Choe S -Y, Wikle III H C and Kim D -J 2008 The design, fabrication and evaluation of a MEMS PZT cantilever with an integrated Si proof mass for vibration energy harvesting J. Micromech. Microeng. 18055017

[23] Liao Y and Sodano H A 2008 Model of a single mode energy harvester and properties for optimal power generation Smart Mater. Struct. 17065026 



\title{
Chapter 5
}

\section{The realization and performance of vibration energy harvesting MEMS devices based on an epitaxial piezoelectric thin film}

\author{
D. Isarakorn ${ }^{1}$, D. Briand ${ }^{1}$, P. Janphuang ${ }^{1}$, A. Sambri ${ }^{2}$, S. Gariglio ${ }^{2}$, J. -M. Triscone ${ }^{2}$, F. Guy ${ }^{3}$, \\ J. W. Reiner ${ }^{4}$, C. H. Ahn ${ }^{4}$ and N. F. de Rooij ${ }^{1}$ \\ ${ }^{1}$ École Polytechnique Fédérale de Lausanne (EPFL), Institute of Microengineering (IMT), \\ The Sensors, Actuators and Microsystems Laboratory, \\ Rue Jaquet-Droz 1, 2002 Neuchâtel, Switzerland \\ ${ }^{2}$ Department of Condensed Matter Physics, University of Geneva, \\ 24 quai Ernest-Ansermet, 1211 Geneva 4, Switzerland \\ ${ }^{3}$ TIN, HEPIA, 4 Rue de la Prairie, CH-1202 Geneva, Switzerland \\ ${ }^{4}$ Department of Applied Physics, Yale University, Becton Center, \\ P.O. Box 208284 New Haven, CT 06520-8284 USA
}

\begin{abstract}
This paper focuses on the fabrication and evaluation of vibration energy harvesting devices by utilizing an epitaxial $\mathrm{Pb}\left(\mathrm{Zr}_{0.2} \mathrm{Ti}_{0.8}\right) \mathrm{O}_{3}(\mathrm{PZT})$ thin film. The high quality of the $c$-axis oriented PZT layer results in a high piezoelectric coefficient and a low dielectric constant, which are key parameters in order to realize high performance piezoelectric energy harvesters. Different cantilever structures, with and without a Si proof mass, are realized using micro-patterning techniques optimized for the epitaxial oxide layers, to maintain the piezoelectric properties throughout the process. The characteristics and the energy harvesting performances of the fabricated devices are experimentally investigated and compared to analytical calculations. The optimized device based on a $0.5 \mu \mathrm{m}$ thick epitaxial PZT film, a cantilever beam of $1 \mathrm{~mm} \times 2.5 \mathrm{~mm} \times 0.015 \mathrm{~mm}$, with a Si proof mass of $1 \mathrm{~mm} \times$ $0.5 \mathrm{~mm} \times 0.23 \mathrm{~mm}$, generates an output power, current and voltage of, respectively, $13 \mu \mathrm{W} g^{-2}, 48 \mu \mathrm{A}$ $g^{-1}$ and $0.27 \mathrm{~V} \mathrm{~g}^{-1}\left(g=9.81 \mathrm{~m} \mathrm{~s}^{-2}\right)$ at the resonant frequency of $2.3 \mathrm{kHz}$ for an optimal resistive load of $5.6 \mathrm{k} \Omega$. The epitaxial PZT harvester exhibits higher power and current with usable voltage, while maintaining lower optimal resistive load if compared with other examples present in the literature. These results indicate the potential of epitaxial PZT thin films for the improvement of the performances of energy harvesting devices.
\end{abstract}




\subsection{Introduction}

Energy harvesting devices are attractive as an energy source for powering micro-devices, such as small wireless sensor networks, biomedical implants, environmental condition monitoring systems and structural health monitoring systems [1-5]. The development of devices able to convert kinetic energy from vibrations, forces or displacements into electric output has advanced rapidly during the past few years because such energy can be found in numerous applications, including industrial machines, transportations, household goods, civil engineering structures, and portable and wearable electronics [3, 6, 7]. Several transduction methods can be used for energy harvesters including electromagnetic induction [8], electrostatic generation [9], and piezoelectric materials [10]. The choice of the transduction methods depends mainly on the applications since there is no clear evidence with respect to the preferred transduction methods [11]. Nevertheless, among these methods, piezoelectric materials have received the most attention due to directly convert vibration energy into electrical energy with a high power density and ease of integration into a system [4, 12, 13], and thus they are well suited to miniaturization. Moreover, harvesters based on piezoelectric materials have a wider operating range at low frequency than the other transduction methods, which can be efficiently utilized to harvest energy from common environmental vibrations [11].

Polycrystalline lead zirconate titanate is the most popular piezoelectric material utilized for energy harvesting applications due to its high piezoelectric coefficient. There are two operation configurations, the $d_{31}$ mode and the $d_{33}$ mode, which are commonly used in piezoelectric harvesters. The $d_{31}$ mode harvesters have a PZT layer in between top and bottom electrodes, while the $d_{33}$ mode harvesters eliminate the need for a bottom electrode by utilizing interdigit electrodes on the top of a PZT layer. Conventionally, bulk piezoelectric harvesters have been developed using the $d_{31}$ mode, which have shown to generate power levels suitable for several applications [10, 12, 14, 15]. However, the use of $d_{31}$ mode for thin-film PZT harvesters often can not provide voltage high enough to activate power management circuits [16]. Therefore, energy harvesters based on PZT thin films employing the $d_{33}$ mode have been developed, compensating the relative low output voltage through the use of a higher piezoelectric coefficient [17] and the optimization of the capacitance by changing the top electrode patterns [18]. Besides PZT thin films, piezoelectric energy harvesters based on aluminum nitride (AlN) thin films have shown an impressive output power of $60 \mu \mathrm{W}$ [19]. Usually, the power is not only governed by the effective piezoelectric coefficient $e$ [20] but also by the dielectric constant $\varepsilon$, being proportional to the ratio $e^{2} / \varepsilon$ [21]. The effective piezoelectric coefficient $e$ can be calculated from the given piezoelectric coefficient, taking into account the elastic modulus and the Poisson ratio of the material. Indeed, although the piezoelectric coefficients of the AlN thin films are lower than those of the PZT thin films, AlN is considered as a candidate material for the energy harvesting applications since the value of $e^{2} / \varepsilon$ for AIN thin films is comparable to the one of 
PZT owing to their lower dielectric constant [19]. While different types of harvesters utilizing polycrystalline films demonstrate interesting power generation levels, the characteristics of the generated voltage and current with respect to the optimal resistive load obtained are different and not always optimum. In general, the electrical characteristics of piezoelectric energy harvesters depend on the properties of the piezoelectric films. Therefore, the type of the piezoelectric films is a key issue to consider in order to improve the overall performance of energy harvesters.

Epitaxial PZT thin films grown on silicon substrates are receiving a lot of interest for many applications because their excellent properties are promising for the realization of novel electronic and MEMS devices [22-25]. In general, epitaxial PZT thin films exhibit piezoelectric and ferroelectric properties superior to polycrystalline films [26]. It is also known that the epitaxial PZT thin films exhibit not only excellent piezoelectric coefficients but also low dielectric constants due to the high $c$ axis orientation $[27,28]$, properties that are known to enhance the performance of piezoelectric energy harvesters, as it was mentioned above. In spite of this positive advancement, the research focused on vibration energy harvesting devices based on epitaxial PZT thin films has been seldom reported [29, 30]. Reilly et al first reported on energy harvesters based on epitaxial thin films grown by pulsed laser deposition [29]. Despite the high quality of the epitaxial films, the power was significantly low $\left.(\sim 1 \mu \mathrm{W} \mathrm{cm})^{-3}\right)$ due to some damage to the piezoelectric film caused by the fabrication process. Recently, Morimoto et al. designed and fabricated energy harvesters composed of epitaxial PZT films transferred onto stainless steel cantilevers to enhance output power efficiency and to improve structural toughness [30]. The transferred epitaxial PZT films exhibited a low dielectric constant and high piezoelectric coefficient, which were able to produce $5.3 \mu \mathrm{W}$ at $0.5 \mathrm{~g}$ acceleration. However, the fabrication throughput, reproducibility and device miniaturization seem to be limited. While the growth of epitaxial piezoelectric thin films on silicon is promising for MEMS based energy harvesters, several challenges still remain for the development of high performance devices based on the epitaxial PZT thin films.

In this paper, we report on the realization and characterization of vibration energy harvesting MEMS devices based on an epitaxial $\mathrm{Pb}\left(\mathrm{Zr}_{0.2} \mathrm{Ti}_{0.8}\right) \mathrm{O}_{3}$ thin film having a high piezoelectric coefficient and a low dielectric constant. The analytical power model and finite element model used to design and optimize the devices, and the choice of the actual PZT stoichiometry for energy harvesting applications are discussed. The results on the fabrication and characterization of the epitaxial PZT cantilevers with and without a Si proof mass are presented. Different characteristics of the fabricated epitaxial PZT harvesters have been studied: their dynamic behavior, electromechanical coupling coefficient and energetic performances. The electrical characteristics of the harvesters, i.e. power, voltage, current and load were experimentally investigated and the results obtained are in good agreement with the analytical model. The performances of the epitaxial PZT harvesters are also compared with other demonstrated energy harvesters. 


\subsection{Principle and design}

\subsubsection{Analytical power model for piezoelectric energy harvesters}

An analytical power model is used for estimating the output power of piezoelectric energy harvesters. Additionally, it is also used for the device design optimization, in order to find the optimal parameters. Many previous researches have developed analytical power models describing the effect of the parameters on the generated output power of the piezoelectric energy harvesters [31-38]. In this work, the analytical power model for a piezoelectric bimorph cantilever with a mass placed on the free end developed by Roundy et al. is applied [12]. This model was derived by combining the standard beam equations with the constitutive equations for a piezoelectric material and a resistive load, defined as:

$$
P_{b}=\frac{1}{2 \omega^{2}} \frac{R_{L} C_{b}^{2}\left(\frac{1.5 E_{P} d_{31}{ }_{p}\left({ }_{p}{ }_{p}+t_{S}\right)}{l_{b}^{2} \varepsilon}\right)^{2} A_{i n}^{2}}{\left(4 \zeta^{2}+k^{2}\right)\left(R_{L} C_{b} \omega\right)^{2}+4 \zeta k^{2}\left(R_{L} C_{b} \omega\right)+4 \zeta^{2}}
$$

where $P_{b}$ : output power of piezoelectric bimorph cantilever, $R_{L}$ : resistive load, $C_{b}$ : capacitance of the PZT film, $E_{p}$ : Young's modulus of the PZT film, $d_{31}$ : piezoelectric coefficient, $t_{p}$ : thickness of PZT, $t_{s}$ : thickness of silicon, $l_{b}$ : length of cantilever, $A_{i n}$ : acceleration of vibration source, $\zeta$ : mechanical damping ratio, $k$ : coupling coefficient, and $\omega$ : the angular frequency of the vibration source, which is equal to the resonant frequency of the cantilever. Our energy harvesting device consists of multilayered epitaxial oxide films deposited on a Si cantilever beam ended with a Si proof mass as shown in figure 5.1. $\mathrm{Si}$ is used as a supporting layer and a proof mass. $\mathrm{SrTiO}_{3}$ (STO) is a buffer layer for the subsequent epitaxial growth. $\mathrm{SrRuO}_{3}(\mathrm{SRO})$ is a metallic oxide film used as a bottom electrode. $\mathrm{Au} / \mathrm{Cr}$ is used as a top electrode and an electrical contact for the bottom electrode. The STO, SRO and Au/Cr layers are much thinner than the thickness of the PZT and Si stack; hence they are omitted in the calculation for simplification. Since the configuration of the energy harvesting device considered in this work is a unimorph cantilever, its output power is a quarter of power obtained from the bimorph structure. Thus, the output power of the unimorph cantilever $(P)$ can be expressed by:

$$
P=\frac{1}{4} P_{b} .
$$


Concerning the resistive load, the main point is that it must be matched to the impedance of the harvester to maximize the output power. The optimal resistive load can be calculated by:

$$
R_{L}=\frac{1}{2 \pi f_{r} C_{b}} \frac{2 \zeta}{\sqrt{4 \zeta^{2}+k^{4}}}
$$

where $f_{r}$ is the resonant frequency. This equation implies that the capacitance of the harvester can adjust the optimal resistive load. Usually, energy harvesting devices with a low optimal load resistance value are highly desirable because their impedance can be easily matched to standard electronic devices. Such harvesters could thus be directly used for electronic devices without an impedance matching circuit resulting in improving the efficiency of the energy harvesting system [39].

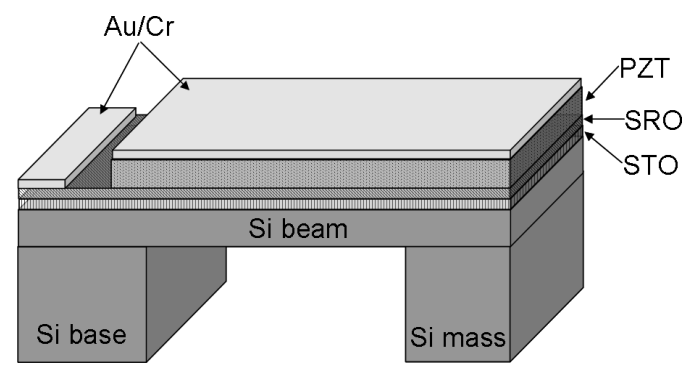

Figure 5.1. Schematic diagram of an epitaxial PZT harvester.

\subsubsection{Resonant frequency}

The frequencies of the ambient vibrations in our environment range from 60 to $200 \mathrm{~Hz}$ [40]. The energy harvesting device needs to be designed to match such low frequency range in order to achieve the maximum output power. In this work, the finite element analysis was performed to predict the resonant frequencies of the devices, since it is a better approach for complex structures compared to analytical model [41, 42]. A three-dimensional finite element model was created with the finite element modeling software ANSYS ${ }^{\circledR}$ (ver. 12.0). Two-layer cantilever structures consisting of a Si layer and an epitaxial PZT thin film with and without a Si mass were modeled. All the others much thinner layers, $\mathrm{Au} / \mathrm{Cr}$, STO and SRO, were not modeled in order to simplify the finite element model. We assume that the thickness of epitaxial PZT layer includes the thickness of the top $\mathrm{Au} / \mathrm{Cr}$ electrode, and the STO and SRO layers are considered as the parts of the Si layer. The material properties listed in table 5.1 and the geometric parameters were introduced into the model. Zero displacement were applied in the $\mathrm{x}, \mathrm{y}$ and $\mathrm{z}$ directions at the one end of the cantilevers. The epitaxial PZT layer was meshed using the SOLID5 coupled-field solid elements, while the Si layer was meshed using the 
SOLID45 3-D structural solid elements. The Block Lanczos method was used to find the resonant frequencies of the devices.

Table 5.1. Material properties used in FEM simulation.

\begin{tabular}{|c|c|c|c|c|c|c|}
\hline$\overline{\text { Material }}$ & $\begin{array}{l}\text { Density } \\
\left(\mathrm{kg} \mathrm{m}^{-3}\right)\end{array}$ & $\begin{array}{l}\text { Young's modulus } \\
\text { (GPa) }\end{array}$ & Poisson's ratio & $\begin{array}{c}\text { Elastic constant } \\
(\mathrm{GPa})\end{array}$ & $\begin{array}{l}\text { Piezoelectric constant } \\
\qquad\left(\mathrm{C} \mathrm{m}^{-2}\right)\end{array}$ & Dielectric constant \\
\hline Silicon [43] & 2330 & 120 & 0.28 & - & - & - \\
\hline Epitaxial PZT $[25,44]$ & 7550 & - & - & $\begin{array}{l}\mathrm{c}_{11}=119.0 \\
\mathrm{c}_{12}=57.9 \\
\mathrm{c}_{13}=56.0 \\
\mathrm{c}_{33}=110.0 \\
\mathrm{c}_{44}=30.5 \\
\mathrm{c}_{66}=30.4\end{array}$ & $\begin{array}{l}\mathrm{e}_{31}=-5.6 \\
\mathrm{e}_{33}=11.3 \\
\mathrm{e}_{15}=7.6\end{array}$ & $\varepsilon_{33}=100$ \\
\hline
\end{tabular}

\subsubsection{Selection of Zr/Ti composition for PZT energy harvesters}

The performance of energy harvesters usually depends on their electrical characteristics. In case of PZT energy harvesters, the electrical characteristics are mainly function of the $\mathrm{Zr} / \mathrm{Ti}$ composition. Most of the PZT energy harvesters utilized the PZT thin films with a stoichiometry near the morphotropic phase boundary (MPB), i.e. around $\mathrm{Zr} / \mathrm{Ti}=53 / 47$, because the piezoelectric coefficients are highest at this composition [45-47]. Nevertheless, the electrical characteristics of the PZT energy harvesters depend not only on the piezoelectric coefficients, but also on the dielectric property. In this study, the electrical characteristics of the PZT energy harvesters with different $\mathrm{Zr} / \mathrm{Ti}$ compositions are therefore evaluated by looking at the effective piezoelectric coefficient $e_{31}$ and the relative dielectric constant $\varepsilon_{r}$. The figures of merit for the various electrical characteristics are summarized in table $5.2[48,49]$.

Table 5.2. Figures of merit for piezoelectric energy harvesters.

\begin{tabular}{lc}
\hline Electrical characteristic & Figure of merit \\
\hline Power generation $\left(\mathrm{P}_{\mathrm{F}}\right)$ & $e_{31}^{2} / \varepsilon$ \\
Voltage generation $\left(\mathrm{V}_{\mathrm{F}}\right)$ & $e_{31} / \varepsilon$ \\
Current generation $\left(\mathrm{I}_{\mathrm{F}}\right)$ & $e_{31}$ \\
\hline
\end{tabular}

Referring to table 5.2, when developing the high performance PZT energy harvesters, it is of critical importance to select the PZT composition, which has high piezoelectric coefficient and low dielectric constant values, in order to obtain the high values of figures of merit. The properties of the 
PZT thin films with different $\mathrm{Zr} / \mathrm{Ti}$ compositions have been widely studied by various research groups [47, 50-52]. Based on these studies, the figures of merit for various electrical characteristics were estimated and plotted in figure 5.2 [52]. It shows a decreasing trend for the power and voltage generation figures of merit when increasing $\mathrm{Zr}$ content of the PZT thin films. On the other hand, the current generation figure of merit increases with increasing $\mathrm{Zr}$ content, and is highest at the composition near the MPB. It should be noted that these figures of merit were estimated from the polycrystalline PZT thin films, whose piezoelectric coefficient is the highest at the MPB and dielectric constant increases with increasing $\mathrm{Zr}$ content [52]. However, the maximum in the piezoelectric coefficient of the well-oriented PZT thin films is often, but not always, observed at the MPB [45, 47, $48,51]$. The piezoelectric coefficient depends on a variety of factors: orientation, grain size, and strain effect. Taking these results into account as design guidelines, the epitaxial thin film with a stoichiometry as $\mathrm{Pb}\left(\mathrm{Zr}_{0.2} \mathrm{Ti}_{0.8}\right) \mathrm{O}_{3}$ is our choice for energy harvesting applications due to the high power and voltage generation figures of merit. It is worth to notice that, although the figure of merit of the output current for this PZT composition is lower than the others, the high degree of crystallographic orientation in the epitaxial films can enhance the piezoelectric coefficients resulting in a higher current generation. Furthermore, an epitaxial thin film with the chosen stoichiometry has a small lattice mismatch with the substrate [53], which can reduce strain effects, and thus further improve the piezoelectric properties [50,54].

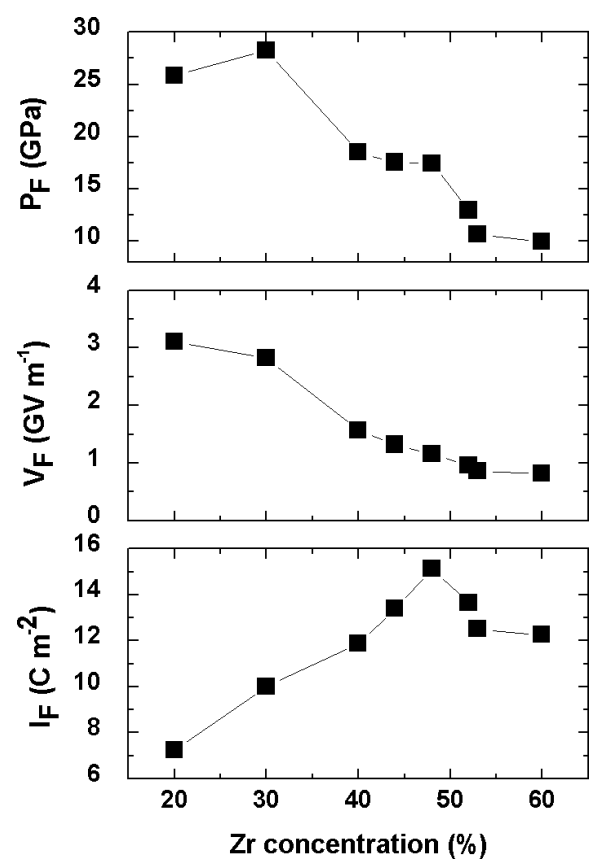

Figure 5.2. Figures of merit as the function of $\mathrm{Zr}$ concentration calculated based on the piezoelectric coefficients and the dielectric constants in [52]. 


\subsection{Fabrication}

\subsubsection{Epitaxial PZT thin films growth on silicon}

The quality of piezoelectric thin films integrated on silicon devices is very important for device performances and reliable use. It is essential to develop a controlled growth procedure to obtain high quality piezoelectric thin films on silicon. The epitaxial growth of piezoelectric thin films is a possible way to integrate high quality piezoelectric films on silicon. Once the difficulties related to the epitaxy of oxides on silicon [55,56] are overcome, epitaxial piezoelectric films present a number of advantages over the textured ones, such as high forces, low driving voltage, and high energyconversion efficiency [50], which allow the realization of MEMS with enhanced actuation/detection properties.

The PZT thin films can be epitaxially grown on silicon through proper intermediate layers. In this work, a 10-20 unit-cell thick $\mathrm{SrTiO}_{3}$ (STO) buffer layer is grown onto 2" $\mathrm{Si}$ (001) wafers by molecular beam epitaxy (MBE) in order to chemically engineer the interface between silicon and the oxide $[57,58]$. This layer represents a good template for the subsequent growth of a metallic $\mathrm{SrRuO}_{3}$ (SRO) film, used as a bottom electrode, grown by off-axis magnetron sputtering. A ferroelectric $\mathrm{Pb}\left(\mathrm{Zr}_{0.2} \mathrm{Ti}_{0.8}\right) \mathrm{O}_{3}$ layer is also grown with the same technique. As mentioned above, such PZT stoichiometry presents a good lattice match with the underlaying SRO layer, an essential requirement for epitaxy [53]. The epitaxial PZT thin films with the thicknesses of $100 \mathrm{~nm}$ and $500 \mathrm{~nm}$ for energy harvesting devices were grown on the SRO $(30 \mathrm{~nm}) / \mathrm{STO}(6 \mathrm{~nm}) / \mathrm{Si}$ substrates. X-ray $\theta-2 \theta$ diffractograms confirm the $c$-axis orientation of the oxide stack and the presence of finite size oscillations around the (001) Bragg peak attests the high crystalline quality of such layers. Rocking curve measurements reveal the good crystalline quality of the piezoelectric layer with a full width half maximum (FWHM) of $0.5^{\circ}$ around the PZT (001) peak.

The polarization and current-voltage measurements were determined on $\mathrm{Cr} / \mathrm{Au}$ test electrodes $\left(100 \times 100 \mu \mathrm{m}^{2}\right)$ using a TF analyzer 2000 system. The responses show clear ferroelectric switching peaks without a significant presence of leakage currents up to $16 \mathrm{~V}$. The measured remnant polarization is of about $70 \mu \mathrm{C} \mathrm{cm}^{-2}$. The epitaxial PZT thin films exhibit a dielectric constant $\varepsilon_{r}$ of $70-100$, characterized by the measurement of the capacitance values on the $\mathrm{Cr} / \mathrm{Au}$ top electrodes of $100 \times 100 \mu \mathrm{m}^{2}$ area. The piezoelectric coefficient $d_{31}$ is evaluated from deflection versus voltage measurements [59], performed on our PZT-based cantilevers with the 100-nm thick PZT layer, and it results to be around 130-140 $\mathrm{pm} \mathrm{V}^{-1}$. A description of the detailed structural and electrical characterization can be found elsewhere [25]. 


\subsubsection{Device fabrication}

The energy harvesting devices consist of multilayered epitaxial oxide films deposited on a silicon cantilever beam with and without a Si proof mass. These devices are realized using micro-patterning techniques optimized for the epitaxial oxide layers deposited on silicon wafers with special emphasis in maintaining the piezoelectric properties of the epitaxial PZT thin films [25]. The process we have developed has been optimized with the right choice of the top electrode material and the minimization of the thermal budget. The process flow is illustrated in figure 5.3. Starting from a heterostructure of $\mathrm{PZT/SRO} / \mathrm{STO}$ grown on $\mathrm{Si}$ as described in the previous section (figure 5.3(a)), $\mathrm{Au}(100 \mathrm{~nm}) / \mathrm{Cr}(10$ $\mathrm{nm})$ square electrodes $(60 \mu \mathrm{m} \times 60 \mu \mathrm{m})$ are deposited through a shadow mask by an e-beam evaporator. These electrodes are used to monitor the variation in the polarization during the progress of the microfabrication process. Contact pads are opened through the epitaxial PZT film to give access to the SRO bottom electrode using a combination of $150 \mathrm{HCl}: 350 \mathrm{H}_{2} \mathrm{O}: 1 \mathrm{HF}$ solution at 55 ${ }^{\circ} \mathrm{C}$, as shown in figure 5.3(b). The shape of the cantilevers is patterned from the top side through the PZT/SRO/STO. This multilayer stack is etched using an Ion Tech ion milling machine (figure 5.3(c)). The back side of the wafer is then etched by deep reactive ion etching (DRIE) to define the thickness of the silicon cantilever (figure 5.3(d)). $\mathrm{An} \mathrm{Au}(100 \mathrm{~nm}) / \mathrm{Cr}(10 \mathrm{~nm})$ layer is evaporated for electrical contacts to the top and bottom electrodes by a lift-off process (figure 5.3(e)). We deposited and patterned the electrodes at the end of the fabrication process in order to minimize the thermal budget during the process to avoid the degradation of the ferroelectric layer $[25,60,61]$. To release the cantilevers from the silicon wafer, a structural release process is performed using frequency-tripled Nd:YAG laser $(\lambda=355 \mathrm{~nm})$ micromachining from the frontside of the wafer (figure 5.3(f)). The polarization was measured to optimize the processing with at the end no degradation of the epitaxial PZT properties throughout the process.

The dimensions of the epitaxial PZT harvesters with different designs are given in table 5.3. For all designs, the epitaxial PZT and Si layers have the same width and length but different thicknesses. The thickness of the Si proof mass is limited by the thickness of the Si substrates. The devices type A (without mass) and B (with mass) were fabricated on the same wafer in order to study the effect of the Si proof mass on the power generation performance, while the device type $\mathrm{C}$ is an optimized structure with a thicker epitaxial PZT layer and a thinner Si cantilever beam. One of the fabricated epitaxial PZT harvesters with the PZT thickness of $500 \mathrm{~nm}$ and the Si proof mass is shown in figure 5.4. 
(a)

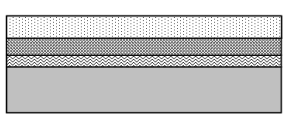

(b)

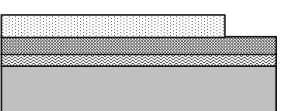

(c)

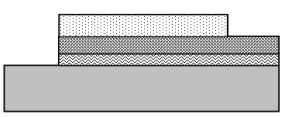

(d)

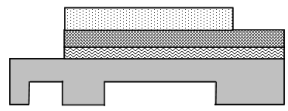

(e)

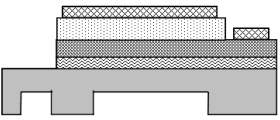

(f)

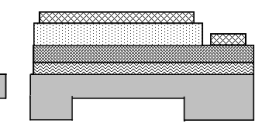

TTO Silicon

Figure 5.3. Schematic of the microfabrication process for the epitaxial PZT harvesters.

Table 5.3. The dimensions of epitaxial PZT harvesters with different designs.

\begin{tabular}{|c|c|c|c|c|c|c|c|}
\hline \multirow{2}{*}{ Device type } & \multirow{2}{*}{ PZT thickness $(\mu \mathrm{m})$} & \multicolumn{3}{|c|}{ Si cantilever beam } & \multicolumn{3}{|c|}{ Si proof mass } \\
\hline & & Width $(\mu \mathrm{m})$ & Length $(\mu \mathrm{m})$ & Thickness $(\mu \mathrm{m})$ & Width $(\mu \mathrm{m})$ & Length $(\mu \mathrm{m})$ & Thickness $(\mu \mathrm{m})$ \\
\hline A & 0.1 & 1000 & 2500 & 30 & - & - & - \\
\hline B & 0.1 & 1000 & 2500 & 30 & 1000 & 500 & 215 \\
\hline $\mathrm{C}$ & 0.5 & 1000 & 2500 & 15 & 1000 & 500 & 230 \\
\hline
\end{tabular}

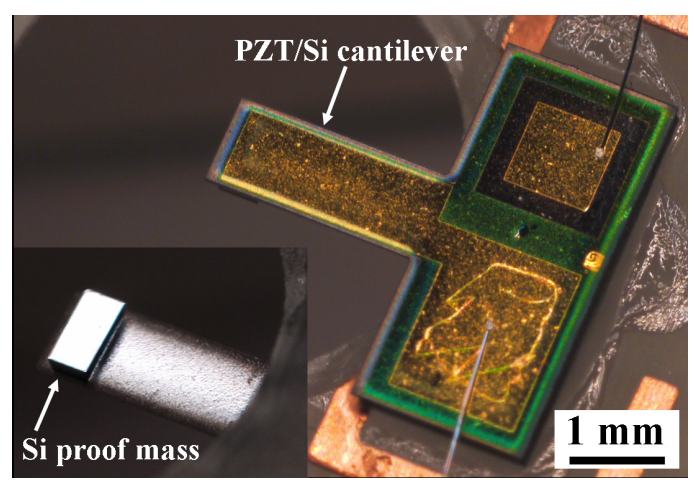

Figure 5.4. Optical image of an epitaxial PZT harvester type $C(1000 \mu \mathrm{m} \times 2500 \mu \mathrm{m} \times 10 \mu \mathrm{m})$ with a Si proof mass $(1000 \mu \mathrm{m} \times 500 \mu \mathrm{m} \times 230 \mu \mathrm{m})$. The inset shows the Si mass on the back side of the harvester.

\subsection{Results and discussion}

The dynamic behavior, the electromechanical coupling coefficient and the energy harvesting performance of the fabricated epitaxial PZT harvesters were characterized. The dynamic characteristics explored here were the resonant frequency, the quality factor and the mechanical 
damping. The electromechanical coupling coefficient is a measure of the effectiveness of a device determined by measuring the frequency response. These measured parameters were introduced to the analytical power model in order to predict the output power. Moreover, they can also be used as the basis for the design optimization. The energy harvesting characteristics, i.e. power, voltage, current and load were then experimentally investigated and compared with the analytical model. Finally, the electrical characteristics of the epitaxial PZT harvester were compared with other demonstrated examples in the literature. All the experiments presented in this section were performed at atmospheric pressure and at room temperature.

\subsubsection{Resonance characterization}

The resonant frequencies of epitaxial PZT cantilevers were characterized using a Polytec MVS-400 laser Doppler vibrometer. The laser beam was positioned at the tip of the epitaxial PZT cantilevers. The epitaxial PZT cantilevers were actuated by a $1 \mathrm{~V}$ amplitude sinusoidal signal with frequencies varying near their simulated resonant frequencies. In figure 5.5, the typical frequency responses of the epitaxial PZT cantilevers with different designs are presented. The resonant frequencies of the devices type $\mathrm{A}, \mathrm{B}$ and $\mathrm{C}$ are found at $9.3 \pm 0.3,4.3 \pm 0.2$ and $2.3 \pm 0.1 \mathrm{kHz}$, respectively. The resonant frequency decreases when the thickness of Si layer is reduced as well as with the addition of a proof mass at the end of the beam. In table 5.4, the measured resonant frequencies (mean values) are compared with the simulated ones. The dimensions of the epitaxial PZT cantilevers used in the simulation are set to match with the ones of the fabricated cantilevers. The measure values are slightly lower than the simulated values. This observed discrepancy could be mainly due to differences between the design and the final geometry of the deposited layers and the Si cantilevers.

The resonant frequencies of these devices were obviously higher than common environmental vibrations $(60-200 \mathrm{~Hz})$ because of the small proof mass limited by the thickness of the Si wafers used (2" in diameter) and the thicker Si cantilever beam. The later is a crucial factor that must be precisely controlled to obtain a desired resonant frequency. We expect that the next design using the silicon-oninsulator technology would lower the resonant frequency to reach the frequency range of the environmental vibrations. 


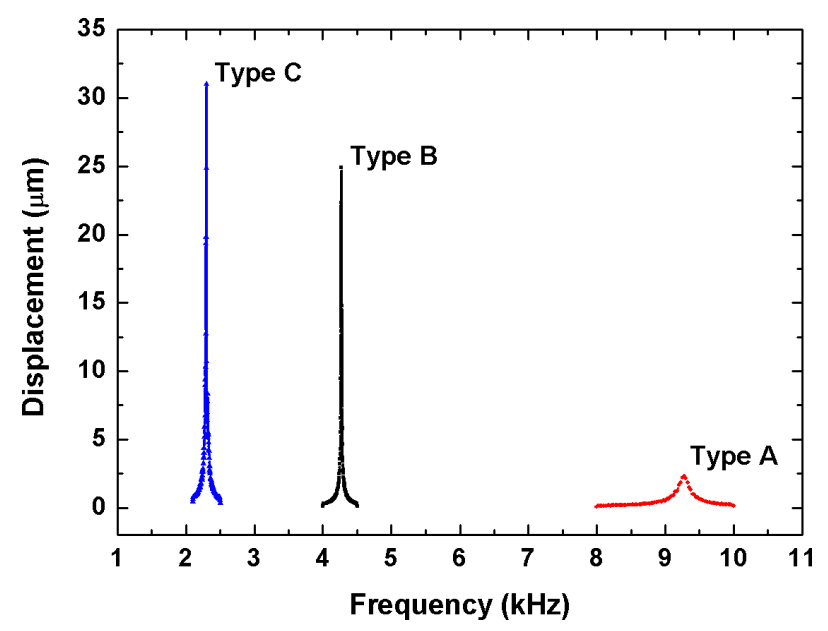

Figure 5.5. Typical first resonant peaks for energy harvesting devices with different designs.

Table 5.4. Comparison of simulated and experimental values of resonant frequencies for devices with different designs.

\begin{tabular}{lccc}
\hline Device type & A & B & C \\
\hline Simulated values $(\mathrm{kHz})$ & 9.439 & 4.306 & 2.324 \\
Experimental values (kHz) & 9.275 & 4.272 & 2.297 \\
Deviation (\%) & 1.7 & 0.8 & 1.2 \\
\hline
\end{tabular}

In energy harvesting applications, the quality factor ( $Q$ factor) is another important parameter that reflects the power generation performance. The $Q$ factor is defined as the ratio between the resonant frequency $\left(f_{r}\right)$ and the width of resonant peak at its half height $(\Delta f)$, i.e. $Q=f_{r} / \Delta f$. Basically, the energy harvesters having a higher $Q$ factor will have a larger deflection. The generated power is proportional to the maximum deflection, so consequently proportional to the $Q$ factor. The $Q$ factors of the epitaxial PZT cantilevers are extracted from the frequency responses in figure 5.5 and found to be 65,138 and 363 for devices type A, B and C, respectively.

\subsubsection{Electromechanical coupling coefficient}

The electromechanical coupling coefficient $k^{2}$ of piezoelectric MEMS devices is a parameter commonly used as a measure of device efficiency. In general, the coupling coefficient $k^{2}$ is defined as the energy transfer from a mechanical (electrical) system to an electrical (mechanical) system. The coupling coefficient $k^{2}$ can be determined by measuring frequencies at resonance $\left(f_{r}\right)$ and antiresonance $\left(f_{a}\right)$, defined as follows: 


$$
k^{2}=1-\left(\frac{f_{r}}{f_{a}}\right)^{2}
$$

The resonant and antiresonant frequencies of epitaxial PZT harvesters were measured on a Hewlett Packard HP 4194A impedance/gain-phase analyzer. The values of coupling coefficients $k^{2}$ of different epitaxial PZT cantilevers derived from the difference between the resonant and antiresonant peaks are shown in table 5.5. It should be noted that the coupling coefficient $k^{2}$ is not the material constant value of epitaxial PZT layer, but depend on the geometry and other elastic materials $[12,17]$.

Table 5.5. The coupling coefficients $k^{2}$ of the epitaxial PZT harvesters.

\begin{tabular}{lccc}
\hline Device type & A & B & C \\
\hline Coupling coefficient $k^{2}$ & $0.041 \pm 0.005$ & $0.053 \pm 0.003$ & $0.056 \pm 0.002$ \\
\hline
\end{tabular}

\subsubsection{Mechanical damping ratio}

In vibratory system, mechanical damping is the effect that energy dissipates during oscillation. The loss of energy results in the decay of amplitude of the oscillations. Basically, output power of energy harvesters is proportional to the deflection. Therefore, low damping effect is highly desirable to maximize the power generation. The damping ratio $(\zeta)$ is a parameter describing the level of damping in a system and defined as the ratio of the damping constant $(c)$ to the critical damping constant $\left(c_{c}\right)$. The damping ratio $(\zeta)$ of epitaxial PZT cantilevers was experimentally determined on the laser Doppler vibrometer using the half-power bandwidth method [62]. This approach is very simple for a quantitative measure of damping level from frequency domain, which is very good for single degree of freedom systems with small values of damping. Several measurements were conducted on each device. The damping ratio $(\zeta)$ can be estimated using the following equation:

$$
\zeta=\frac{f_{2}-f_{1}}{2 f_{r}}
$$

where $f_{2}-f_{1}$ is frequency difference at which the amplitude is 0.707 times the resonant amplitude. Table 5.6 illustrates the values of the damping ratios with respect to different designs of the epitaxial PZT cantilevers. 
Table 5.6. The damping ratios $\zeta$ of the epitaxial PZT harvesters.

\begin{tabular}{lccc}
\hline Device type & A & B & C \\
\hline Damping ratio $\zeta$ & $0.0077 \pm 0.0023$ & $0.0008 \pm 0.0001$ & $0.0004 \pm 0.0001$
\end{tabular}

\subsubsection{Energy harvesting performance}

The energy harvesting performance of the epitaxial PZT harvesters has been investigated with a shaker (Bruel \& Kjaer type 4811) driven by a vibration exciter control (type 1050) and a power amplifier (type 2712), by applying an acceleration as a mechanical input. The harvesters placed inside a metallic box for noise protection were mounted on the shaker. The harvesters were connected with various resistive loads $R_{L}$, and the ac current $I_{r m s}$ generating through a resistive load under different acceleration levels was recorded with a multimeter (Agilent 34411A). The schematics of the experimental setup and the epitaxial PZT harvester are shown in figure 5.6. The generated voltage from the devices was calculated by Ohm's Law. The corresponding average power is calculated by:

$$
P_{a v e}=I_{r m s}^{2} R_{L} .
$$
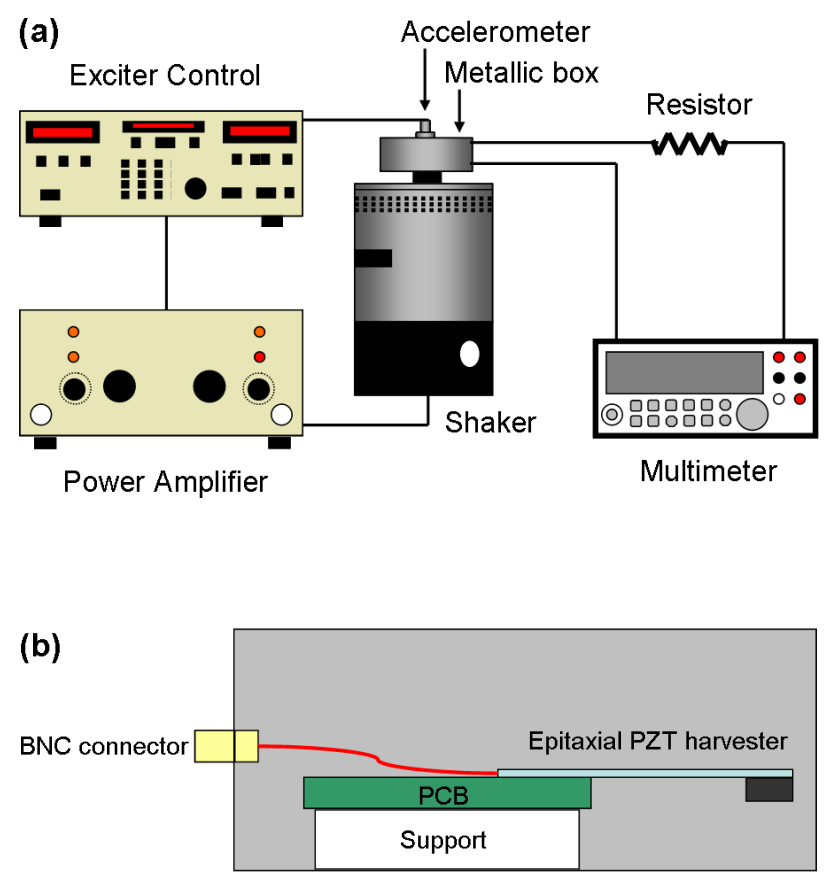

Figure 5.6. Schematics of (a) the experimental setup for output power characterization and (a) the epitaxial PZT harvester mounted inside the metallic box for noise protection. 
The devices type A and B were excited under different acceleration levels ranging from $0.1 \mathrm{~g}$ to $1 \mathrm{~g}$ at their resonant frequencies in order to compare the performance of the devices with and without a Si proof mass. The $g$ is the gravitational acceleration, where $1 g$ implies $9.81 \mathrm{~m} \mathrm{~s}^{-2}$. The theoretical and experimental values of the current generated through the various load resistors are shown in figure 5.7. The calculated power and voltage are also shown in figure 5.8 and 5.9, respectively. The theoretical results were obtained by introducing the experimental mean values of the coupling coefficients $k^{2}$, the damping ratios $\zeta$, the resonant frequency $f_{r}$, and the piezoelectric coefficient $d_{31}$ in equations (5.1) and (5.2). These output data were normalized per square acceleration $\left(g^{2}\right)$ for output power and per acceleration $(g)$ for current and voltage. The normalized values are used to quantify the absolute power, current, and voltage generated by an energy harvester at a given acceleration level. As expected, the device with the Si proof mass (type B) can generate higher power than the device without the Si proof mass (type A) because of the higher stress on the epitaxial PZT layer induced by the Si proof mass. The maximum output power was found to be 0.34 $\mathrm{nW} g^{-2}$ at the optimal resistive load of $220 \Omega$ for the device type A and $161 \mathrm{nW} g^{-2}$ at the optimal resistive load of $330 \Omega$ for the device type B. At the optimal resistive loads, the device type A produced $1.2 \mu \mathrm{A} \mathrm{g}^{-1}$ and $0.27 \mathrm{mV} \mathrm{g}^{-1}$, while the device type B produced $22 \mu \mathrm{Ag}^{-1}$ and $7.3 \mathrm{mV} \mathrm{g}^{-1}$. For both devices, the experimental results agree well with the theoretical values predicted by equation (5.1). However, there is some variability in the experimental values that resulted in the large error bars. This could be mainly due to the variations of the material properties and of the Si thicknesses as well as the changes of the elastic compliance of the PZT thin films due to nonlinear effects under large stress at high acceleration levels $[63,64]$.

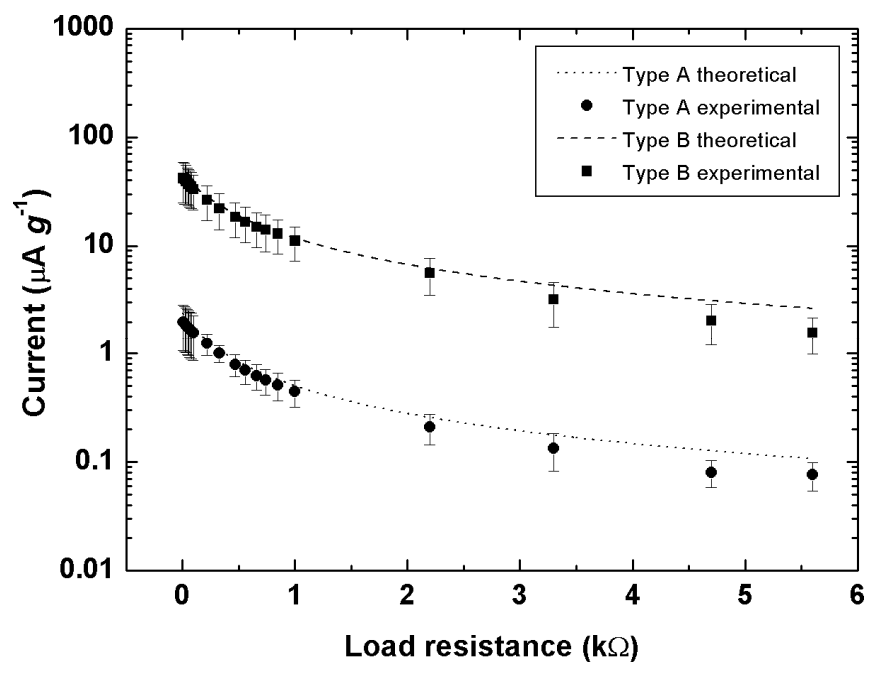

Figure 5.7. Theoretical and experimental currents versus load resistance for the devices type A and type B. 

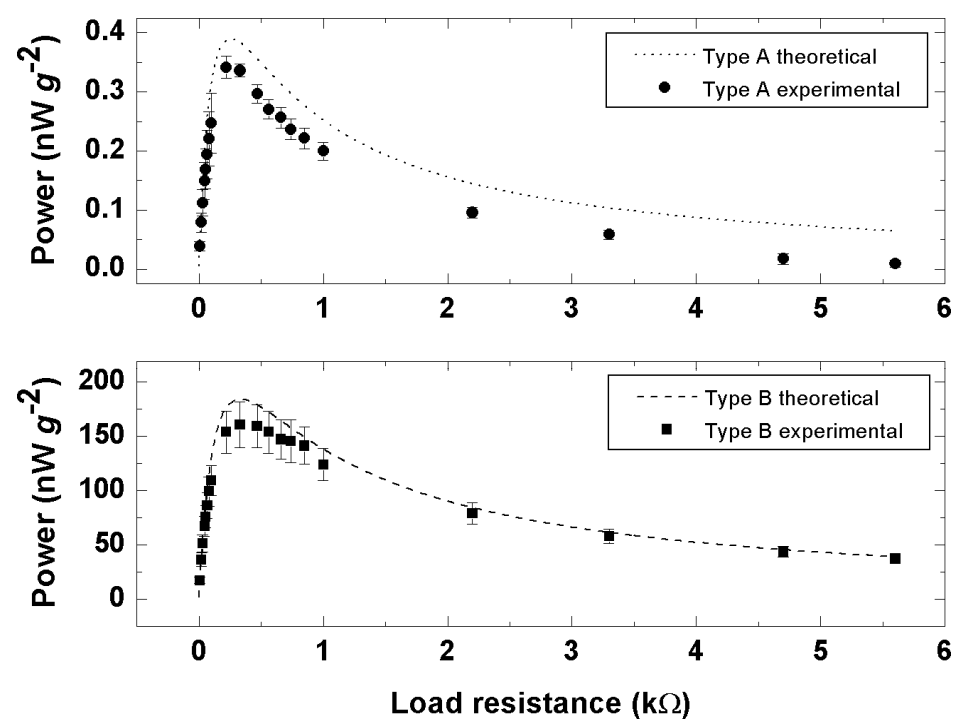

Figure 5.8. Theoretical and experimental powers versus load resistance for the devices type A (upper) and type B (lower).

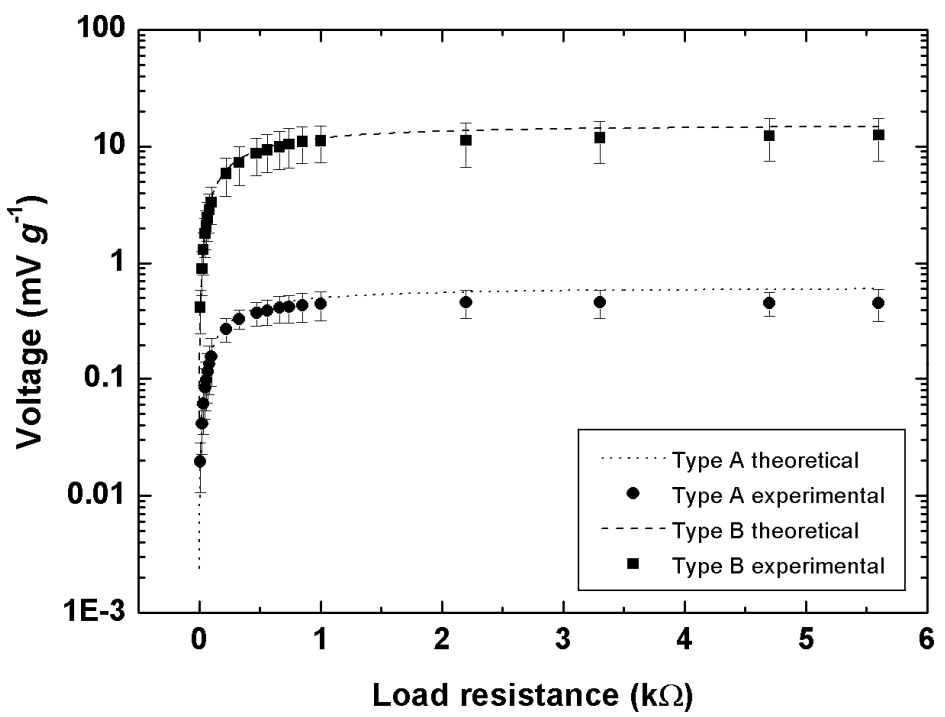

Figure 5.9. Theoretical and experimental voltages versus load resistance for the devices type A and type B.

The device type $\mathrm{C}$ was then characterized by the same experimental procedures. The normalized values of the output current, average power and voltage as a function of resistive loads at different acceleration levels are shown in figure 10. A maximum output power of $13 \mu \mathrm{W} g^{-2}$ with 48 $\mu \mathrm{A} g^{-1}$ output current was obtained for an optimal resistive load of $5.6 \mathrm{k} \Omega$, and thus resulting in the 
output voltage of $0.27 \mathrm{~V} \mathrm{~g}^{-1}$. The theoretical results were also plotted in figure 5.10. The experimental results almost followed the theoretical calculations, but the experimental value of the optimal resistive load was found to be higher than the theoretical one. This discrepancy could be due to an overestimation of the capacitance value of the epitaxial PZT thin films due to the variations of either the dielectric constant or the thickness of the epitaxial PZT thin film.
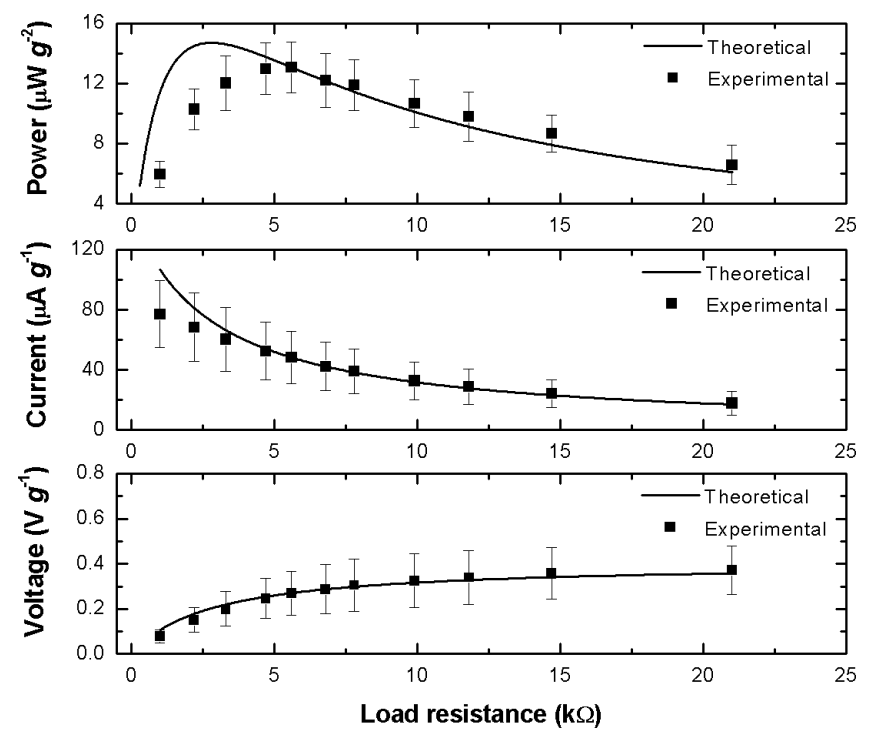

Figure 5.10. Theoretical and experimental power, current and voltage values versus load resistance for the device type $\mathrm{C}$.

The experimental results of the epitaxial PZT harvesters with different designs are summarized in table 5.7. As adding the Si proof mass and decreasing the thickness of the Si cantilever beam, the resonant frequency was reduced significantly, and the power generation was also largely enhanced. The power density was calculated using the normalized power divided by the volume of the Si beam cantilever and the Si proof mass, which is the so-called effective volume. The power density is used to compare the performance of different energy harvesters irrespective of device size. The power density values of these three devices were $0.005 \mu \mathrm{W} g^{-2} \mathrm{~mm}^{-3}$ (type A), $0.879 \mu \mathrm{W} g^{-2} \mathrm{~mm}^{-3}$ (type B), and $85 \mu \mathrm{W} \mathrm{g}{ }^{-2} \mathrm{~mm}^{-3}$ (type C). Such a high power density of the device type $\mathrm{C}$ is of great interest for the realization of miniaturized energy harvesting MEMS devices. The optimal resistive load is generally inversely proportional to the capacitance of the piezoelectric materials. The devices type A and $\mathrm{B}$ have higher capacitance values than the device type $\mathrm{C}$ due to the thinner epitaxial PZT films. Therefore, the optimal resistive load for the device type $\mathrm{C}$ was larger than the devices type $\mathrm{A}$ and $\mathrm{B}$. Usually, energy harvesting devices with a low optimal resistive load are highly desirable because their impedance can be easily matched to standard electronic devices [39]. Overall, the device type C optimized with the thicker epitaxial PZT layer and the thinner Si cantilever beam enables the highest 
electric generation performance among the other designs. Although it required the higher optimal resistive load, this property can be further improved by changing the PZT composition in order to modify the dielectric constant value.

Table 5.7. Output performance of the epitaxial PZT harvesters.

\begin{tabular}{|c|c|c|c|c|c|c|c|}
\hline Device type & $\begin{array}{c}\text { Resonant frequency } \\
(\mathrm{kHz})\end{array}$ & $\begin{array}{c}\text { Power } \\
\left(\mu \mathrm{W} g^{-2}\right)\end{array}$ & $\begin{array}{l}\text { Current } \\
\left(\mu \mathrm{A}^{-1}\right)\end{array}$ & $\begin{array}{l}\text { Voltage } \\
\left(\mathrm{mV}^{-1}\right)\end{array}$ & $\begin{array}{c}\text { Optimal load } \\
(\Omega)\end{array}$ & $\begin{array}{l}\text { Effective volume } \\
\qquad\left(\mathrm{mm}^{3}\right)\end{array}$ & $\begin{array}{l}\text { Power density } \\
\left(\mu \mathrm{W} \mathrm{g} \mathrm{gm}^{-3}\right)\end{array}$ \\
\hline $\bar{A}$ & 9.275 & 0.00034 & 1.2 & 0.27 & 220 & 0.075 & 0.005 \\
\hline B & 4.272 & 0.161 & 22 & 7.3 & 330 & 0.183 & 0.879 \\
\hline $\mathrm{C}$ & 2.297 & 13 & 48 & 270 & 5600 & 0.153 & 85 \\
\hline
\end{tabular}

\subsubsection{A comparison of piezoelectric energy harvesting devices}

For different types of piezoelectric energy harvesters, many designs utilize polycrystalline PZT and AIN films. In this section, we compare the electrical characteristics, power, voltage, current and load of piezoelectric harvesters made of different materials and film textures. It should be noted that the comparison presented here were estimated from the data given in the references. Therefore, they should only be used as a guideline. Figure 5.11 compares the normalized power and power density of different types of selected piezoelectric energy harvesting devices. In figure 5.11(a), the epitaxial PZT harvester produced the output power slightly lower than the state-of-the-art energy harvester based on the AlN film [19]. It is worth to notice, anyway, that the higher impedance exhibited from energy harvesting devices based on polycrystalline AlN films limits their output current. Epitaxial PZT harvester exhibited the best power density over other harvesters as shown in figure 5.11(b). Besides the power generating performance, the other electrical characteristics such as current, voltage and load are also specified in table 5.8. Overall, the harvester based on the epitaxial PZT film produces superior performances, higher current and smaller resistive load due to excellent piezoelectric properties. A very interesting outcome is that the epitaxial PZT harvester generates higher power and current with usable voltage, and requires lower optimal resistive load compared to piezoelectric harvesters realized with polycrystalline PZT or AlN thin films. These results indicate the potential of epitaxial PZT thin films for the energy harvesting applications, which can improve the overall performance of energy harvesting devices. 

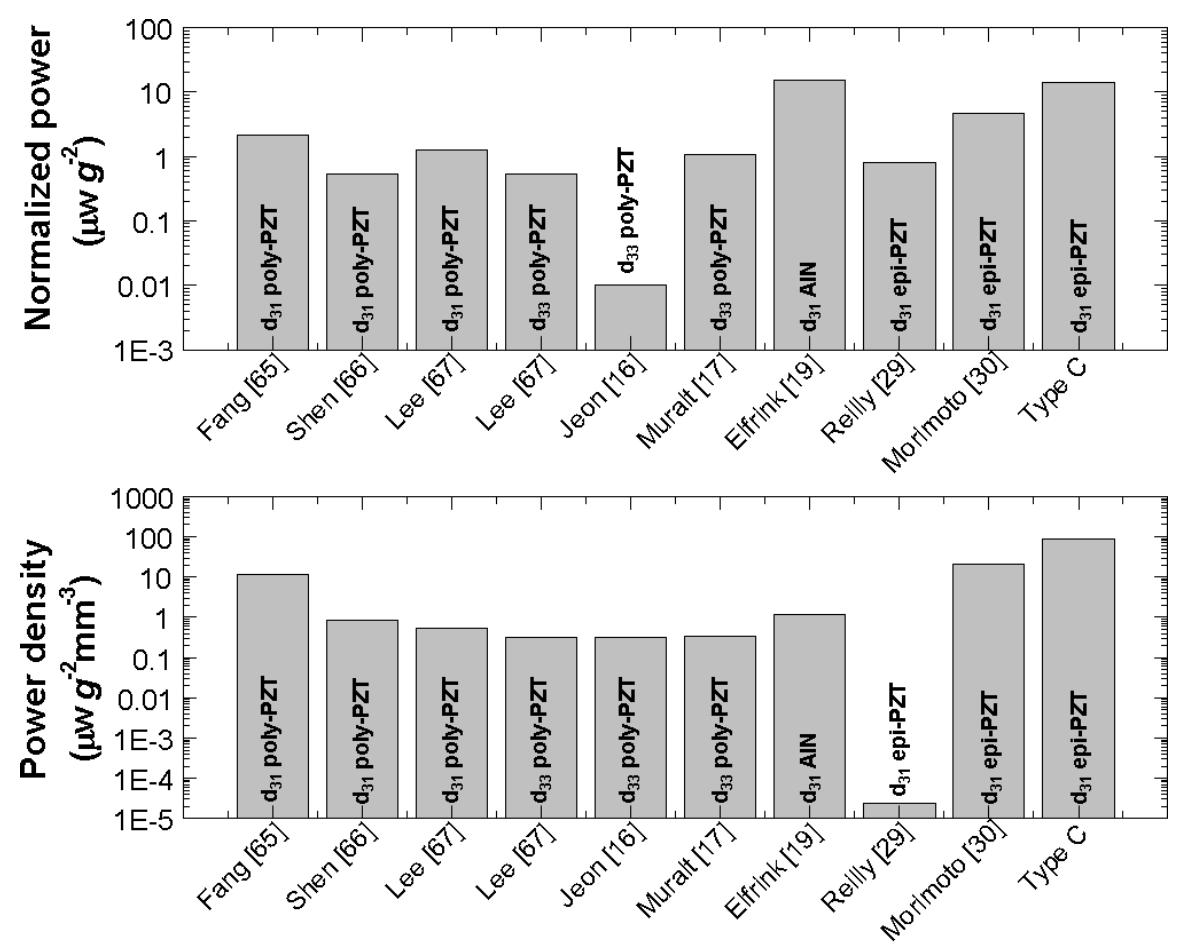

Piezoelectric energy harvester

Figure 5.11. Comparison of energy harvesting performances for different piezoelectric energy harvesters: (a) normalized power and (b) power density. Note that $d_{31}$ and $d_{33}$ refer to the operating modes, poly-PZT represents polycrystalline PZT, AIN represents aluminum nitride, and epi-PZT represents epitaxial PZT.

Table 5.8. Energy harvesting performances of different piezoelectric energy harvesting devices.

\begin{tabular}{lccccccc}
\hline Harvester & Type & $\begin{array}{c}\text { Piezo thickness Effective volume } \\
(\mu \mathrm{m})\end{array}$ & $\left(\mathrm{mm}^{3}\right)$ & $\left(\mathrm{V}^{-1} \mathrm{~mm}^{-3}\right)$ & $\left(\mu \mathrm{A}^{-1} \mathrm{~mm}^{-3}\right)$ & $\begin{array}{c}\text { Frequency } \\
(\mathrm{Hz})\end{array}$ & $\begin{array}{c}\text { Optimal load } \\
(\mathrm{k} \Omega)\end{array}$ \\
\hline Fang [65] & $d_{31}$ poly-PZT & 1.64 & 0.1944 & 1.059 & 49.516 & 608 & 21.4 \\
Shen [66] & $d_{31}$ poly-PZT & 1 & 0.6520 & 0.087 & 14.570 & 462.5 & 6 \\
Lee [67] & $d_{31}$ poly-PZT & 5 & 0.4245 & 1.869 & 12.462 & 256 & 150 \\
Lee [67] & $d_{33}$ poly-PZT & 5 & 0.6120 & 1.872 & 3.672 & 214 & 510 \\
Jeon [16] & $d_{33}$ poly-PZT & 0.48 & 0.0270 & 7.852 & 1.518 & 13900 & 5200 \\
Muralt [17] & $d_{33}$ poly-PZT & 2 & 0.3248 & 2.463 & 2.694 & 870 & 1800 \\
Elfrink [19] & $d_{31}$ AlN & 0.8 & 12.7272 & 0.204 & 0.454 & 572 & $100-1000$ \\
Reilly [29] & $d_{31}$ epi-PZT & 1 & 0.0000296 & 0.005 & $9.8 \times 10^{-6}$ & 971 & 510000 \\
Morimoto [30] & $d_{31}$ epi-PZT & 2.8 & 4.625 & 0.795 & 15.890 & 126 & 50 \\
Type C & $d_{31}$ epi-PZT & 0.5 & 0.1530 & 1.76 & 313.73 & 2297 & 5.6 \\
\hline
\end{tabular}




\subsection{Conclusions}

This paper addressed the realization and performance of energy harvesters by utilizing an epitaxial $\mathrm{Pb}\left(\mathrm{Zr}_{0.2} \mathrm{Ti}_{0.8}\right) \mathrm{O}_{3}$ thin film for vibration energy harvesting applications. The epitaxial PZT thin film grown on a Si substrate through oxide layers exhibits a high piezoelectric coefficient and a low dielectric constant, characteristics that made it a promising material for piezoelectric energy harvesting devices. Different types of epitaxial PZT cantilevers, with and without a Si proof mass, have been successfully fabricated through optimized micro-patterning techniques. Electrical characteristics, i.e., generated current, voltage, power and load of the epitaxial PZT harvesters were theoretically and experimentally evaluated: the experimental results are in very good agreement with the theoretical ones. The optimized epitaxial PZT harvester produced a power, current and voltage of, respectively, $13 \mu \mathrm{W} \mathrm{g} g^{-2}, 48 \mu \mathrm{A} g^{-1}$ and $0.27 \mathrm{~V} \mathrm{~g}^{-1}$ at its resonant frequency of $2.3 \mathrm{kHz}$ for an optimal resistive load of $5.6 \mathrm{k} \Omega$. Compared to the other piezoelectric energy harvesters surveyed in the literature, the power and current density values of the epitaxial PZT harvester developed in this study were largest, while maintaining the lowest optimal resistive load. Based on the results of this study, the epitaxial PZT thin film has a high potential for vibration energy harvesting applications. The next points to be addressed are the lowering the resonant frequency through an improvement of the structure design based on the use of the silicon-on-insulator technology and a study of the power management circuits in order to realize fully-functional vibration energy harvesting systems based on this technology.

\section{Acknowledgements}

This work was supported by the Swiss National Science Foundation through the National Centre of Competence in Research 'Materials with Novel Electronic Properties' MaNEP and by the European Union through the project 'Nanoxide'. The work at Yale was supported by NSF MRSEC

DMR 0520495 and the FENA center. Don Isarakorn is grateful to the Ministry of Science and Technology, Thailand for the financial support for his PhD studies at EPFL IMT-NE SAMLAB. 


\section{References}

[1] Beeby S P, Torah R N, Tudor M J, Glynne-Jones P, O’Donnell T, Saha C R and Roy S 2007 A micro electromagnetic generator for vibration energy harvesting J. Micromech. Microeng. 17 1257-65

[2] Harb A 2010 Energy harvesting: State-of-the-art Renewable Energy 1-14 (article in press)

[3] Beeby S P, Tudor M J and White N M 2006 Energy harvesting vibration sources for Microsystems applications Meas. Sci. Technol. 17 R175-R195

[4] Anton S R and Sodano H A 2007 A review of power harvesting using piezoelectric materials (2003-2006) Smart Mater. Struct. 16 R1-R21

[5] Vullers R J M, van Schaijk R, Doms I, Van Hoof C and Mertens R 2009 Micropower energy harvesting Solid-State Electron. 53 684-93

[6] Elvin N G, Lajnef N and Elvin A A 2006 Feasibility of structural monitoring with vibration powered sensors Smart Mater. Struct. 15 977-86

[7] Granstrom J, Feenstra J, Sodano H A and Farinholt K 2007 Energy harvesting from a backpack instrumented with piezoelectric shoulder straps Smart Mater. Struct. 16 1810-20

[8] Glynne-Jones P, Tudor M J, Beeby S P and White N M 2004 An electromagnetic, vibrationpowered gernerator for intelligent sensor systems Sens. Actuators, A $110344-9$

[9] Peano F and Tambosso T 2005 Design and optimization of a MEMS electret-based capacitive energy scavenger J. Microelectromech. Syst. 14 435-529

[10] Shen D, Choe S -Y and Kim D -J 2007 Analysis of piezoelectric materials for energy harvesting devices under high-g vibrations Jpn. J. Appl. Phys. 46 6755-60

[11] Mitcheson P D, Reilly E K, Toh T, Wright P K and Yeatman E M 2007 Performance limits of the three MEMS inertial energy generator transduction types J. Micromech. Microeng. 17 S211-S216

[12] Roundy S and Wright P K 2004 A piezoelectric vibration based generator for wireless electronics Smart Mater. Struct. 13 1131-42

[13] Muralt P, Polcawich R G and Trolier-McKinstry S 2009 Piezoelectric thin films for sensors, actuators, and energy harvesting MRS Bull. 35 658-64

[14] Zheng Q and Xu Y2008 Asymmetric air-spaced cantilevers for vibration energy harvesting Smart Mater. Struct. 17055009

[15] Sodano H A, Inman D J and Park G 2005 Comparison of piezoelectric energy harvesting devices for recharging batteries J. Intell. Mater. Syst. Struct. 16 799-807

[16] Jeon Y B, Sood R, Jeong J -H and Kim S -G 2005 MEMS power generator with transverse mode thin film PZT Sens. Actuators, A 122 16-22

[17] Muralt P, Marzencki M, Belgacem B, Calame F and Basrour S 2009 Vibration energy harvesting with PZT micro device Procedia Chemistry 1 1191-4 
[18] Zhang Q Q, Gross S J, Tadigadapa S, Jackson T N, Djuth F T and Trolier-McKinstry S 2003 Lead zirconate titanate films for $d_{33}$ mode cantilever actuators Sens. Actuators, A 105 91-7

[19] Elfrink R, Kamel T M, Goedbloed M, Matova S, Hohlfeld D, van Andel Y and van Schaijk R 2009 Vibration energy harvesting with aluminum nitride-based piezoelectric devices $J$. Micromech. Microeng. 19094005

[20] Muralt P 1997 Piezoelectric thin films for MEMS Integr. Ferroelectr. 17 297-307

[21] Van Schaijk R, Elfrink R, Kamel T M and Goedbloed M 2008 Piezoelectric AlN energy harvesters for wireless autonomous transducer solutions Proc. of IEEE Sensors 2008, (Lecce, Italy) 45-8

[22] Mathews S, Ramesh R, Venkatesan T and Benedetto J 1997 Ferroelectric field effect transistor based on epitaxial perovskite heterostructures Science $\mathbf{2 7 6} 238-40$

[23] Haertling G H 1991 Ferroelectric thin films for electronic applications J. Vac. Sci. Technol., A 9 414-20

[24] Nguyen M D, Nazeer H, Karakaya K, Pham S V, Steenwelle R, Dekkers M, Abelmann L, Blank D H A and Rijnders G 2010 Characterization of epitaxial $\mathrm{Pb}(\mathrm{Zr}, \mathrm{Ti}) \mathrm{O}_{3}$ thin films deposited by pulsed laser deposition on silicon cantilevers J. Micromech. Microeng. 20 085022

[25] Isarakorn D, Sambri A, Janphuang P, Briand D, Gariglio S, Triscone J -M, Guy F, Reiner J W, Ahn C H, de Rooij N F 2010 Epitaxial piezoelectric MEMS on silicon J. Micromech. Microeng. 20055008

[26] Ramesh R and Schlom D G 2002 Orienting Ferroelectric Films Science 296 1975-6

[27] Kanno I, Fujii S, Kamada T and Takayama R 1997 Piezoelectric properties of $c$-axis oriented $\mathrm{Pb}(\mathrm{Zr}, \mathrm{Ti}) \mathrm{O}_{3}$ thin films Appl. Phys. Lett. 70 1378-80

[28] Kanno I, Kotera H and Wasa K 2003 Measurement of transverse piezoelectric properties of PZT thin films Sens. Actuators, A 107 68-74

[29] Reilly E K and Wright P K 2009 Modeling, fabrication and stress compensation of an epitaxial thin film piezoelectric microscale energy scavenging device J. Micromech. Microeng. 19095014

[30] Morimoto K, Kanno I, Wasa Kiyotaka and Kotera H 2010 High-efficiency piezoelectric energy harvesters of $c$-axis-oriented epitaxial PZT films transferred onto stainless steel cantilevers Sens. Actuators, A 163 428-32

[31] Kim M, Hoegen M, Dugundji J and Wardle B L 2010 Modeling and experimental verification of proof mass effects on vibration energy harvester performance Smart Mater. Struct. 19 045023 
[32] Li Y, Li W, Guo T, Yan Z, Fu X and Hu X 2009 Study on structure optimization of a piezoelectric cantilever with a proof mass for vibration-powered energy harvesting system $J$. Vac. Sci. Technol., B 27 1288-90

[33] Lu F, Lee H P and Lim S P 2004 Modeling and analysis of micro piezoelectric power generators for micro-electromechanical-systems applications Smart Mater. Struct. 13 57-63

[34] Erturk A and Inman D J 2008 Issues in mathematical modeling of piezoelectric energy harvesters Smart Mater. Struct. 17065016

[35] Chen S -N, Wang C -J and Chien M -C 2006 Analytical modeling of piezoelectric vibrationinduced micro power generator Mechatronics 16 379-87

[36] Shu Y C and Lien I C 2006 Analysis of power output for piezoelectric energy harvesting systems Smart Mater. Struct. 15 1499-512

[37] Ajitsaria J, Choe S Y, Shen D and Kim D J 2007 Modeling and analysis of a bimorph piezoelectric cantilever beam for voltage generation Smart Mater. Struct. 16 447-54

[38] Renaud M, Karakaya K, Sterken T, Fiorini P, Van Hoof C and Puers R 2008 Fabrication, modeling and characterization of MEMS piezoelectric vibration harvesters Sens. Actuators, A

145-146 380-6

[39] Song H -C, Kim H -C, Kang C -Y, Kim H -J, Yoon S -J and Jeong D -Y 2009 Multilayer piezoelectric energy scavenger for large current generation J. Electroceram. 23 301-4

[40] Roundy S, Wright P K and Rabaey J 2003 A study of low level vibrations as a power source for wireless sensor nodes Comput. Commun. 26 1131-44

[41] Wang C, Wang Z, Ren T -L, Zhu Y, Yang Y, Wu X, Wang H, Fang H and Liu L 2007 A micromachined piezoelectric ultrasonic transducer operating in $d_{33}$ mode using square interdigital electrodes IEEE Sens. J. 7 967-76

[42] Defay E, Millon C, Malhaire C and Barbier D 2002 PZT thin films integration for the realization of a high sensitivity pressure microsensor based on a vibrating membrane Sens. Actuators, A 99 64-7

[43] Luginbuhl Ph, Racine G -A, Lerch Ph, Romanowicz B, Brooks K G, de Rooij N F, Renaud Ph and Setter N 1996 Piezoelectric cantilever beams actuated by PZT sol-gel thin film Sens. Actuators, A $\mathbf{5 4}$ 530-5

[44] 1990 Landolt-Börnstein: Numerical Data and Functional Relationships in Science and Technology New serie, Group III, vol 26 (Berlin: Springer-Verlag) p 123

[45] Dubois M -A and Muralt P 1999 Measurement of the effective transverse piezoelectric coefficient $e_{31, f}$ of $\mathrm{AlN}$ and $\mathrm{Pb}\left(\mathrm{Zr}_{\mathrm{x}}, \mathrm{Ti}_{1-\mathrm{x}}\right) \mathrm{O}_{3}$ thin films Sens. Actuators, $A$ 77 106-12

[46] Ledermann N, Muralt P, Baborowski J, Gentil S, Mukati K, Cantoni M, Seifert A and Setter $\mathrm{N} 2003\{100\}$-Textured, piezoelectric $\mathrm{Pb}\left(\mathrm{Zr}_{\mathrm{x}}, \mathrm{Ti}_{1-\mathrm{x}}\right) \mathrm{O}_{3}$ thin films for MEMS: integration, deposition and properties Sens. Actuators, A 105 162-70 
[47] Kim D -J, Maria J -P, Kingon A I and Streiffer S K 2003 Evaluation of intrinsic and extrinsic contributions to the piezoelectric properties of $\mathrm{Pb}\left(\mathrm{Zr}_{\mathrm{x}} \mathrm{Ti}_{1-\mathrm{x}}\right) \mathrm{O}_{3}$ thin films as a function of composition J. Appl. Phys. 93 5568-75

[48] Trolier-McKinstry S and Muralt P 2004 Thin film piezoelectric for MEMS J. Electroceram. 12 7-17

[49] Wasa K, Kanno I and Kotera H 2009 Fundamentals of thin film piezoelectric materials and processing design for a better energy harvesting MEMS Proc. PowerMEMS 200961

[50] Wang Y, Ganpule, Liu B T, Li H, Mori K, Hill B, Wuttig M, Ramesh R, Finder J, Yu Z, Droopad R and Eisenbeiser K 2002 Epitaxial ferroelectric $\mathrm{Pb}(\mathrm{Zr}, \mathrm{Ti}) \mathrm{O}_{3}$ thin films on Si using $\mathrm{SrTiO}_{3}$ template layers Appl. Phys. Lett. 80 97-9

[51] Kanno I, Kotera H, Wasa K, Matsunaga T, Kamada T and Takayama R 2003 Crystallographic characterization of epitaxial $\mathrm{Pb}(\mathrm{Zr}, \mathrm{Ti}) \mathrm{O}_{3}$ films with different $\mathrm{Zr} / \mathrm{Ti}$ ratio grown by radio-frequency-magnetron sputtering J. Appl. Phys. 93 4091-6

[52] Hoffmann M, Kuppers H, Schneller T, Bottger U, Schnakenberg U, Mokwa W and Waser R 2003 Theoretical calculations and performance results of a PZT thin film actuator IEEE Trans. Ultrason., Ferroelect., Freq. Contr. 501240

[53] Gariglio S, Stucki N, Triscone J -M and Triscone G 2007 Strain relaxation and critical temperature in epitaxial ferroelectric $\mathrm{Pb}\left(\mathrm{Zr}_{0.20} \mathrm{Ti}_{0.80}\right) \mathrm{O}_{3}$ thin films Appl. Phys. Lett. 90202905

[54] Kuffer O, Maggio-Aprile I, Triscone J -M, Fischer O and Renner Ch 2000 Piezoelectric response of epitaxial $\mathrm{Pb}\left(\mathrm{Zr}_{0.20} \mathrm{Ti}_{0.80}\right) \mathrm{O}_{3}$ films measured by scanning tunneling microscopy Appl. Phys. Lett. 77 1701-3

[55] Lin A, Hong X, Wood V, Verevkin A A, Ahn C H, McKee R A, Walker F J and Specht E D 2001 Epitaxial growth of $\mathrm{Pb}\left(\mathrm{Zr}_{0.2} \mathrm{Ti}_{0.8}\right) \mathrm{O}_{3}$ on $\mathrm{Si}$ and its nanoscale piezoelectric properties Appl. Phys. Lett. 78 2034-36

[56] Dekkers M, Nguyen M D, Steenwelle R, te Riele P M, Blank D H A and Rijnders G 2009 Ferroelectric properties of epitaxial $\mathrm{Pb}(\mathrm{Zr}, \mathrm{Ti}) \mathrm{O}_{3}$ thin films on silicon by control of crystal orientation Appl. Phys. Lett. 95012902

[57] Reiner J W, Garrity K F, Walker F J, Ismail-Beigi S and Ahn C H 2008 Role of strontium in oxide epitaxy on silicon (001) Phys. Rev. Lett. 101105503

[58] Su D, Yang B, Jiang N, Sawicki M, Broadbridge C, Couillard M, Reiner J W, Walker F J, Ahn C H and Zhu Y 2010 Valence electron energy-loss spectroscopy of ultrathin $\mathrm{SrTiO}_{3}$ films grown on silicon (100) single crystal Appl. Phys. Letter. 96121914

[59] Smits J and Choi W 1991 The constituent equations of piezoelectric heterogeneous bimorphs IEEE Trans. Ultrason. Ferroelectr. Freq. Contr. 38 256-70

[60] Shimamoto $\mathrm{Y}$, Kushida-Abdelghafar K, Miki H and Fujisaki Y $1997 \mathrm{H}_{2}$ damage of ferroelectric $\mathrm{Pb}(\mathrm{Zr}, \mathrm{Ti}) \mathrm{O}_{3}$ thin-film capacitors-The role of catalytic and adsorptive activity of the top electrode Appl. Phys. Lett., 70 3096-7 
[61] Nagaraj B, Aggarwal S and Ramesh R 2001 Influence of contact electrodes on leakage characteristics in ferroelectric thin films J. Appl. Phys. 90, 375-82

[62] Olmos B A and Roesset J M 2010 Evaluation of the half-power bandwidth method to estimate damping in systems without real modes Earthquake Engng Struct. Dyn. DOI: $10.1002 /$ eqe. 1010

[63] Wang Q -M, Zhang Q, Xu B, Liu R and Cross L E 1999 Nonlinear piezoelectric behavior of ceramic bending mode actuators under strong electric fields J. Appl. Phys. 86, 3352-60

[64] Yao L Q, Zhang J G, Lu L and Lai M O 2004 Nonlinear dynamic characteristics of piezoelectric bending actuators under strong applied electric field J. Microelectromech. Syst. $13645-52$

[65] Fang H -B, Liu J -Q, Xu Z -Y, Dong L, Wang L, Chen D, Cai B -C and Liu Y 2006 Fabrication and performance of MEMS-based piezoelectric power generator for vibration energy harvesting Microelectron. J. 37 1280-4

[66] Shen D, Park J -H, Ajitsaria J, Choe S -Y, Wikle III H C and Kim D -J 2008 The design, fabrication and evaluation of a MEMS PZT cantilever with an integrated Si proof mass for vibration energy harvesting J. Micromech. Microeng. 18055017

[67] Lee B S, Lin S C, Wu W J, Wang X Y, Chang P Z and Lee C K 2009 Piezoelectric MEMS generators fabricated with an aerosol deposition PZT thin film J. Micromech. Microeng. 19 065014 



\title{
Chapter 6
}

\section{Finite element analysis and experiments on a silicon membrane actuated by an epitaxial PZT thin film for localized-mass sensing applications}

\author{
D. Isarakorn ${ }^{1}$, D. Briand ${ }^{1}$, A. Sambri ${ }^{2}$, S. Gariglio ${ }^{2}$, J. -M. Triscone ${ }^{2}$, F. Guy ${ }^{3}$, \\ J. W. Reiner ${ }^{4}$, C. H. Ahn ${ }^{4}$, N. F. de Rooij ${ }^{1}$ \\ ${ }^{1}$ École Polytechnique Fédérale de Lausanne (EPFL), Institute of Microengineering (IMT), \\ The Sensors, Actuators and Microsystems Laboratory, \\ Rue Jaquet-Droz 1, P.O. Box 526, 2002 Neuchâtel, Switzerland \\ ${ }^{2}$ Department of Condensed Matter Physics, University of Geneva, \\ 24 quai Ernest-Ansermet, 1211 Geneva 4, Switzerland \\ ${ }^{3}$ TIN, HEPIA, 4 Rue de la Prairie, CH-1202 Geneva, Switzerland \\ ${ }^{4}$ Department of Applied Physics, Yale University, \\ Becton Center, P.O. Box 208284 New Haven, CT 06520-8284 USA
}

\begin{abstract}
In this paper, we investigate the performance of a piezoelectric membrane actuated by an epitaxial piezoelectric $\mathrm{Pb}\left(\mathrm{Zr}_{0.2} \mathrm{Ti}_{0.8}\right) \mathrm{O}_{3}$ (PZT) thin film for localized-mass sensing applications. The fabrication and characterization of piezoelectric circular membranes based on epitaxial thin films prepared on a silicon wafer are presented. The dynamic behavior and mass sensing performance of the proposed structure are experimentally investigated and compared to numerical analyses. A $1500 \mu \mathrm{m}$ diameter silicon membrane actuated by a $150 \mathrm{~nm}$ thick epitaxial PZT film exhibits a strong harmonic oscillation response with a high quality factor of 110-144 depending on the resonant mode at atmospheric pressure. Different aspects related to the effect of the mass position and of the resonant mode on the mass sensitivity as well as the minimum detectable mass are evaluated. The operation of the epitaxial PZT membrane as a mass sensor is determined by loading polystyrene microspheres. The mass sensitivity is a function of the mass position, which is the highest at the antinodal points. The epitaxial PZT membrane exhibits a mass sensitivity in the order of $10^{-12} \mathrm{~g} \mathrm{~Hz}^{-1}$ with a minimum detectable mass of $5 \mathrm{ng}$. The results reveal that the mass sensor realized with the epitaxial PZT thin film, which is capable of generating a high actuating force, is a promising candidate for the
\end{abstract}


development of high performance mass sensors. Such devices can be applied for various biological and chemical sensing applications. 


\subsection{Introduction}

Mass sensors have attracted interest as a sensing platform for biomolecular and chemical detection. A great amount of research has been dedicated to the development of high performance mass sensors suitable for measuring masses down to molecular or atomic levels [1-10]. Mass sensors can be classified according to their operating principles as static and dynamic mode devices. In the static mode, the deflection of sensors due to surface stress caused by mass loading is measured as the output signal. In the dynamic mode, the mass microbalancing technique is adopted to measure the change in resonant frequency, which reflects the change in mass loading. Several types of microstructures including microcantilever, micromembrane, and microbridge have been investigated for mass sensing applications in the dynamic mode $[1,4,5]$. Among these microstructures, the resonant microcantilevers actuated by piezoelectric thin films have received the most attention in the field because of their high sensitivity, real-time detection capability, and simplicity [1, 10]. However, they are limited by their fragility under fabrication processes and real operating conditions, and they also suffer from a low quality factor in the operation in viscous liquid [1]. Moreover, an extra passivation layer on the cantilever is required to electrically insulate the electrodes and the piezoelectric layer during the operation in a liquid media, which often degrades the performance of the sensors [11].

To overcome these issues, several piezoelectric membrane sensors have been successfully demonstrated for mass sensing applications [1, 10, 12-14]. The membrane structures are advantageous compared to the cantilevers for three different reasons. First, the membrane structures are more rigid than cantilevers, thus minimizing the structural fragility problem. Second, the top surface of membrane structures is physically isolated from the bottom surface [11]. Thus, they can be easily adapted to be used in liquid solutions without requiring an additional passivation layer [9]. Third reason, the quality factor of the membrane is not effected by the variation of liquid viscosity within certain limits [12]. Therefore, the membrane structures could be a promising candidate for the mass sensing applications.

Beside the study related to the choice of an appropriate geometry, the properties of the piezoelectric films employed in the mass sensors represent a crucial factor that need to be investigated in order to improve the performances of such devices [15]. Ideally, the piezoelectric films must generate a high actuating force and large displacement in order to obtain a high quality factor, which is related to the minimum mass a sensor can detect. Typically, in the mass sensors, piezoelectric $\mathrm{Pb}(\mathrm{Zr}, \mathrm{Ti}) \mathrm{O}_{3}$ (PZT) thin films are preferred to $\mathrm{AlN}$ and $\mathrm{ZnO}$ films, because of their superior piezoelectric properties. The use of PZT thin films for mass detection has already shown promising performances. However, the forces generated by PZT thin films, whose thickness is around $1 \mu \mathrm{m}$, are commonly too small to overcome the high effective mass in the viscous environment [16]. It have been reported that the mass sensor realized with about $22 \mu \mathrm{m}$ thick PZT film demonstrated a very good quality factor due to an excellent harmonic oscillation response [16]. In spite of this positive advancement, it should be taken into account that for the integration of PZT thick films on Si 
substrates, high processing temperature is required in order to obtain high density and good electromechanical properties. Moreover, the requirement of higher actuation voltages for PZT thick films with respect to the thinner is not suitable for realizing integration systems with an electronic read-out circuit [17]. It is evident that improving the actuating force of PZT thin films is one of the most important challenges in the development of high performance mass sensors.

Epitaxial PZT thin films on Si have attracted a lot of interest because their properties are promising for the realization of novel MEMS devices [18]. Indeed, epitaxial piezoelectric films exhibit properties, including piezoelectric coefficients, polarization, and dielectric constant superior to polycrystalline films [19]. Although the growth of epitaxial piezoelectric films on silicon is a challenging process, it can be achieved by mean of appropriate transition oxide layers. Recently, we have demonstrated the ability to grow very high quality epitaxial $\mathrm{Pb}\left(\mathrm{Zr}_{0.2} \mathrm{Ti}_{0.8}\right) \mathrm{O}_{3}$ (PZT) thin film on silicon, with rms roughness of about $5 \mathrm{~nm}$ over $1 \mu \mathrm{m}^{2}$ and very large piezoelectric coefficients. Cantilever and membrane transducers realized with $100 \mathrm{~nm}$ thick PZT films exhibits a strong harmonic oscillation at a low actuation voltage with a high quality factor in dynamic mode [20]. Taking these results into account, there is a great potential to use such epitaxial devices for mass sensing applications as well.

In this study, we explore the application of the circular silicon membrane actuated by the epitaxial piezoelectric PZT thin film for a localized-mass detection. The fabrication and characterization of the epitaxial PZT membranes are presented. The focus is the exploration of different capabilities of the epitaxial PZT membrane as a mass sensor for localized-mass detection at atmospheric pressure. The mass sensing performance of the epitaxial device is investigated both numerically and experimentally. The operating characteristics of the epitaxial PZT membranes as a mass sensor are evaluated by loading polystyrene microspheres. The finite element model of the epitaxial PZT membrane is developed to determine the resonant frequency, the effect of the position of the mass and of the resonant mode on the mass sensitivity. Finite element analyses of different modes of resonances agree within a few percent deviations with the experimental values. We also report on the dependence of the mass sensitivity on the mass position and on the vibration mode, being highest at the antinodes of vibrations. The experimentally observed change in resonant frequency is of the order $10^{-12} \mathrm{~g} \mathrm{~Hz}^{-1}$, which is in excellent agreement with the simulated value. Our best minimum detectable mass measured using an optical technique based on laser vibrometry corresponds to $\sim 5 \mathrm{ng}$. These results indicate the performance of the epitaxial PZT membrane that can be applied for various mass sensing applications.

\subsection{Mass sensing performance -theoretical background}

Mass sensing based on piezoelectric actuation is a powerful label-free technique that is receiving attention for many applications, ranging from biomedical, chemical, biosensors, and even atomic 
physics [6]. The mass sensing behavior can be explained by the performance parameters, such as the mass sensitivity, the limit of detection or the minimum detectable mass, and the quality factor. These parameters are of considerable importance when developing a high performance mass sensor. In this section, the key performance parameters are introduced, and each of these parameters will be discussed in details in the following subsections.

\subsubsection{Mass sensitivity}

In the view of mass sensor development, the mass sensitivity is an important characteristic representing the sensor performance. A definition of mass sensitivity is the variation in a measurable parameter as a function of added mass on the sensing area. The measurable parameter commonly used for mass sensors operating in the resonant mode is the mechanical resonance, where the resonant frequency change $\Delta f$ of the sensor is proportional to the added mass $\Delta m$. Thus, the mass sensitivity can be defined as:

$$
\frac{\Delta m}{\Delta f}=\mathfrak{R}^{-1}=-\frac{2 m_{e f f}}{f_{0}}
$$

where $\mathfrak{R}$ is the mass responsivity, $m_{\text {eff }}$ is the effective mass of sensor at a certain resonant mode, and $f_{0}$ is the initial resonant frequency before mass loading [21]. From the above equation, a lower absolute value of $\mathfrak{R}^{-1}$ means that the sensor is more sensitive. The mass sensitivity can be increased by decreasing the sensor mass. This can be achieved by either using higher order mode of resonance to reduce the effective mass of the sensor or directly reducing the size of the sensor [22].

\subsubsection{Limit of detection}

Another important performance metric of a mass sensor is the ability to detect the smallest amount of mass. This performance metric is commonly characterized by the limit of detection (LOD). The LOD is determined using the mass sensitivity together with the noise level of the sensor signal [23, 24], given by:

$$
\delta m=\delta f \Re^{-1}
$$

where $\delta m$ is the minimum detectable mass change, and $\delta f$ is the fluctuation level of the resonant frequency around its peak. Since the mass sensitivity is defined by the dimensions and the material properties of the membrane sensor as given by equation (6.1), the minimum detectable mass mainly 
pertains to the resonant frequency fluctuation. Therefore, depressing the resonant frequency fluctuation will improve the LOD of the sensor.

\subsubsection{Quality factor}

In mass sensing applications, the quality factor ( $Q$ factor) reflects the sensor resolution. The $Q$ factor is defined as the ratio between the resonant frequency $\left(f_{r}\right)$ and the width of resonant peak at its half height $\left(\Delta f_{r}\right)$, expressed as follows:

$$
Q=\frac{f_{r}}{\Delta f_{r}}
$$

A higher $Q$ factor value implies a sharper resonant peak, which results in a higher resolution in determining the change in the resonant frequency. Moreover, the $Q$ factor impacts directly on the resonant frequency fluctuation $[22,25,26]$, which is related to the minimum detectable mass change. Therefore, the $Q$ factor is a key parameter for the performance of mass sensors. The mass sensors with the higher $Q$ factor value are highly desirable due to not only higher resolution but also better signal-to-noise ratio [27].

\subsection{Growth of epitaxial thin films on silicon}

The quality of piezoelectric thin films integrated on silicon devices is very important for device performances and reliable use. The epitaxial growth of piezoelectric thin films is a way to integrate high quality piezoelectric films on silicon.

To grow high quality epitaxial PZT thin films on silicon, proper intermediate layers are required. In this work, a 10-20 unit-cell thick $\mathrm{SrTiO}_{3}$ (STO) buffer layer is grown on 2" $\mathrm{Si}$ (001) wafers by molecular beam epitaxy (MBE) $[28,29]$. Such layer represents a good template for the subsequent growth of a metallic $\mathrm{SrRuO}_{3}$ (SRO) film, used as a bottom electrode, grown by off-axis magnetron sputtering. With the same technique, a ferroelectric $\mathrm{Pb}\left(\mathrm{Zr}_{0.2} \mathrm{Ti}_{0.8}\right) \mathrm{O}_{3}$ layer is successively grown. Such PZT stoichiometry presents a good lattice match with the underlaying SRO layer, essential requirement for epitaxy [30]. The typical final stack results as PZT $(150 \mathrm{~nm}) / \mathrm{SRO}$ (30 $\mathrm{nm}) / \mathrm{STO}(6 \mathrm{~nm}) / \mathrm{Si}$. X-ray $\theta-2 \theta$ diffractograms confirm the $c$-axis orientation of the oxide stack and the presence of finite size oscillations around the (001) Bragg peak attests the high crystalline quality of such layers.

The current-voltage and hysteresis measurements realized on $\mathrm{Cr} / \mathrm{Au}$ test electrodes $(100 \times$ $100 \mu \mathrm{m}^{2}$ ) using a TF analyzer 2000 system show clear ferroelectric switching peaks without a significant presence of leakage currents up to $16 \mathrm{~V}$, indicating the robust dielectric properties of the epitaxial PZT thin film on silicon. The epitaxial PZT films exhibit a dielectric constant $\varepsilon_{r}$ of 70-100 
and a remanent polarization of about $70 \mu \mathrm{C} \mathrm{cm}$. Piezoelectric Force Microscopy (PFM) measurements confirm the out-of-plane orientation of the polarization along the $c$-axis and show a mono-domain configuration for the ferroelectric polarization. The piezoelectric coefficient $d_{31}$ is evaluated from deflection versus voltage measurements, performed on our PZT-based cantilevers, and it results to be as high as $130-140 \mathrm{pm} \mathrm{V}^{-1}$ [31]. It should be noted that the epitaxial PZT films were not subjected to a poling treatment prior to the measurements since they were naturally polarized. A description of the detailed structural and electrical characterization can be found elsewhere [20].

\subsection{Device fabrication}

Circular membranes actuated by the thin epitaxial PZT film are fabricated using micro-patterning techniques optimized for the oxide layers [20]. The structure was made as simple as possible and its process flow is depicted in figure 6.1. Starting from a heterostructure of PZT/SRO/STO grown on Si as described in the previous section (figure 6.1(a)), contact pads are opened through the epitaxial PZT film using a combination of $150 \mathrm{HCl}: 350 \mathrm{H}_{2} \mathrm{O}: 1 \mathrm{HF}$ solution at $55^{\circ} \mathrm{C}$ (figure $6.1(\mathrm{~b})$ ). The back side of the wafer is then etched by DRIE to define the thickness of the silicon membrane (figure 6.1(c)). Subsequently, an $\mathrm{Au}(100 \mathrm{~nm}) / \mathrm{Cr}(10 \mathrm{~nm})$ layer is evaporated as the top electrodes and for electrical contacts of the bottom electrodes by a lift-off process (figure 6.1(d)). Solid circular electrodes are used to electrically excite vibrations in the epitaxial PZT membranes. Since the thickness of silicon layer is much greater than the epitaxial PZT thickness, this electrode configuration produces more deflection compared to concentric rings, spirals and segmented electrodes [32]. Each chip shown in Figure 6.2 , whose size is $5 \mathrm{~mm} \times 5 \mathrm{~mm}$, is glued on a printed circuit board and the connections are made with wire bonding. The measured dimension of the circular PZT membrane is $1500 \mu \mathrm{m} \pm 5 \mu \mathrm{m}$ in diameter. The silicon thickness is $15 \mu \mathrm{m} \pm 2 \mu \mathrm{m}$. The dimensions of the device are designed based primarily on the resonant frequency determination. Our device is designed to operate in a resonant frequency range of $90-370 \mathrm{kHz}$ [20], which allows us to use simple and inexpensive measurement equipment [1].

(a)

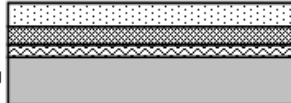

(b)
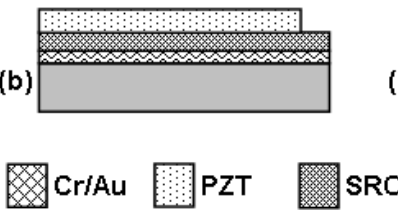

(c)

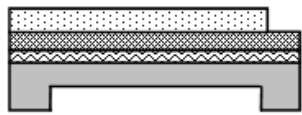

(d)
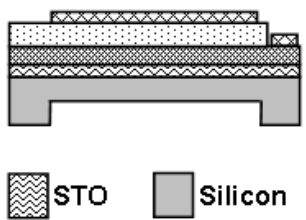

Figure 6.1. Schematic of the microfabrication process for an epitaxial PZT membrane: (a) epitaxial multilayer deposition; (b) bottom electrode opening via wet etching; (c) Si membrane patterning by DRIE; (d) top electrode and electrical contact patterning by liftoff. 


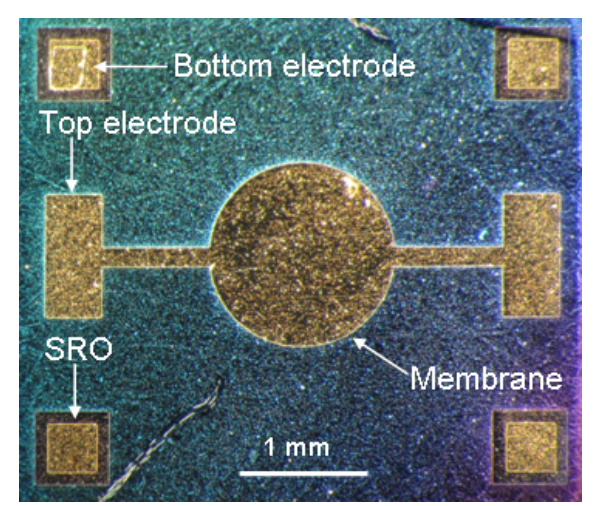

Figure 6.2. Optical image of an epitaxial PZT membrane.

\subsection{Finite element analysis}

Finite element model has been extensively used as a tool to design and develop complex micro devices [33-37], since it is a better approach for determining the performance of such devices compared to analytical model. In this work, the finite element analysis is performed to determine the characteristics of the epitaxial PZT membrane, e.g. its resonant behavior and its resonant frequency shift due to the added mass at various positions along the membrane radius. A three-dimensional finite element model was created with the finite element modeling software ANSYS $^{\circledR}$ (ver. 12.0). The material properties and the geometric parameters were introduced into the model. The finite element model consists of the following three layers: a silicon membrane, an epitaxial PZT thin film, and a top electrode. The STO and SRO layers are not modeled since their thicknesses are much smaller than the thickness of the silicon membrane. Therefore, they are considered to be the parts of the silicon membrane in order to simplify the finite element model. Similarly, the thin $\mathrm{Cr}$ adhesive layer is included in the Au layer. The diameter of the circular PZT membrane used in the simulation is 1500 $\mu \mathrm{m}$. The thicknesses of silicon, PZT and Au layers are $15 \mu \mathrm{m}, 150 \mathrm{~nm}$, and $100 \mathrm{~nm}$, respectively. Zero displacements are applied in the $\mathrm{x}, \mathrm{y}$ and $\mathrm{z}$ directions at the boundaries as illustrated in figure 6.3. The piezoelectric coefficient $d_{31}$ was derived from the displacement at the free end of the epitaxial PZT cantilever beam structure [20]. The permittivity of the epitaxial PZT thin film was characterized by the measurement of the capacitance values on the $\mathrm{Cr} / \mathrm{Au}$ top electrodes of $100 \times 100 \mu^{2}$ area. Material properties for the silicon [38], the epitaxial PZT [20, 39], and the Au [40] layers are listed in table 6.1 


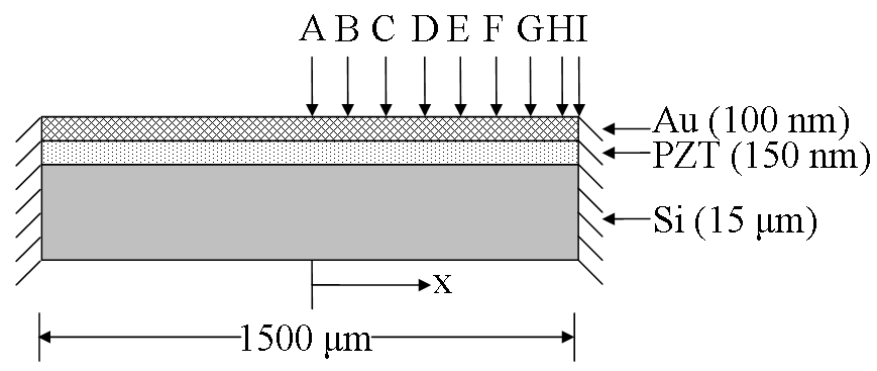

Figure 6.3. Schematic (side view) of boundary conditions and the mass position along the radius of the epitaxial PZT membrane in the modeling. Positions A, B, C, D, E, F, G, H, and I are at $\mathrm{x}=0,100$, $200,300,400,500,600,700$, and $750 \mu \mathrm{m}$ from the center of the membrane, respectively.

Table 6.1. Material properties for finite element analysis.

\begin{tabular}{|c|c|c|c|c|c|c|}
\hline Material & $\begin{array}{l}\text { Density } \\
\left(\mathrm{kg} \mathrm{m}^{-3}\right)\end{array}$ & $\begin{array}{c}\text { Young's modulus } \\
\text { (GPa) }\end{array}$ & Poisson's ratio & $\begin{array}{c}\text { Elastic constant } \\
\text { (GPa) }\end{array}$ & $\begin{array}{l}\text { Piezoelectric constant } \\
\qquad\left(\mathrm{C} \mathrm{m}^{-2}\right)\end{array}$ & Dielectric constant \\
\hline Silicon & 2330 & 120 & 0.28 & - & - & - \\
\hline \multirow[t]{6}{*}{ Epitaixial PZT } & 7550 & - & - & $\mathrm{c}_{11}=119.0$ & $e_{31}=-5.6$ & $\varepsilon_{33}=100$ \\
\hline & & & & $c_{12}=57.9$ & $e_{33}=11.3$ & \\
\hline & & & & $\mathrm{c}_{13}=56.0$ & $e_{15}=7.6$ & \\
\hline & & & & $c_{33}=110.0$ & & \\
\hline & & & & $c_{44}=30.5$ & & \\
\hline & & & & $c_{66}=30.4$ & & \\
\hline $\mathrm{Au}$ & 19300 & 78 & 0.35 & - & - & - \\
\hline Polystylene & 1050 & 3.6 & 0.34 & - & - & - \\
\hline
\end{tabular}

\subsubsection{Resonant frequency simulation}

A modal analysis was carried out to determine the vibrational characteristics, including the resonant frequencies and modal shapes of the epitaxial PZT membranes. The epitaxial PZT layer was meshed using the SOLID98 tetrahedral coupled-field solid elements. The other layers including the silicon and Au layers were meshed using the SOLID95 3-D 20-node structural solid elements. The Block Lanczos method was chosen for finding the resonant frequencies. Figure 6.4 shows the modal shapes corresponding to the first four resonant frequencies of the $1500 \mu \mathrm{m}$ diameter membrane with the color indicating the displacement of the membrane, where red and blue represent the antinode and node of vibrations. The resonant frequencies of symmetrical vibration modes corresponding to the 01- and 02modes were found at 94.11 and $367.43 \mathrm{kHz}$, while those of asymmetrical vibration modes were at 196.23 and $322.79 \mathrm{kHz}$ for 11- and 21-modes, respectively. 

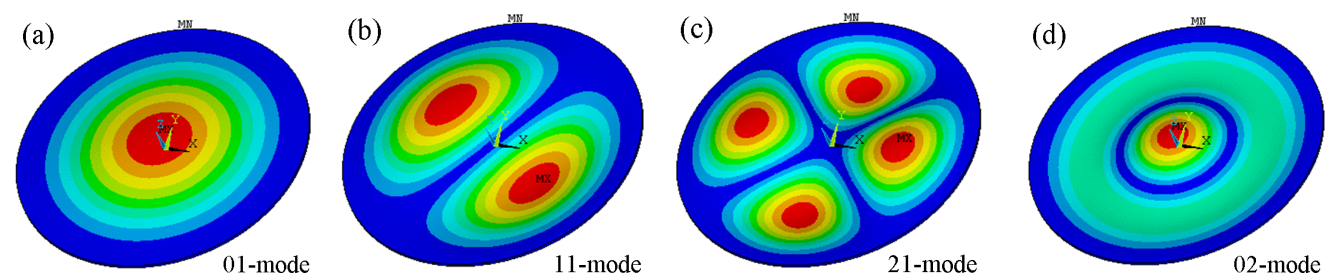

Figure 6.4. Modal shapes of the epitaxial PZT membrane obtained through a finite element analysis with the color indicating the displacement of the membrane (red=antinode; blue=node). The resonances appeared at (a) $94.11 \mathrm{kHz}$ for 01 -mode, (b) $196.23 \mathrm{kHz}$ for 11 -mode, (c) $322.79 \mathrm{kHz}$ for 21-mode, and (d) $367.43 \mathrm{kHz}$ for 02 -mode.

\subsubsection{Mass-change sensitivity}

The mass-change sensitivity characteristics of the epitaxial PZT membrane are studied to determine the optimum sensitivity as a function of the mass position and the vibration mode. The mass loading can be performed with a localized mass or a distributed mass. It has been reported that the sensitivity for a localized-mass sensor is better than that for a distributed-mass sensor [41-43]. Moreover, adding mass locally can avoid stiffening of the sensor structure due to the much smaller contact area between the sensing surface and mass [43]. Thus, the mass sensing performance is investigated by adding a point-mass to the epitaxial PZT membrane along the radius direction of the membrane and examining the resonant frequency for several modes. The polystyrene microsphere (used as a mass in the experiments) was modeled as a polystyrene box for simplification. The length of the polystyrene box ranges from $24 \mu \mathrm{m}$ to $66 \mu \mathrm{m}$ depending on the desired weight. The weight of the polystyrene box was set to match with the one of the polystyrene microsphere. The mechanical properties of polystyrene are shown in table 6.1 .

The effect of mass loading (72.2 $\mathrm{ng}$ ) at various positions on the resonant frequency of all four modes is examined for the epitaxial PZT membrane with a diameter of $1500 \mu \mathrm{m}$. The positions of the added mass are illustrated in figure 6.3, where A, B, C, D, E, F, G, H, and I are $\mathrm{x}=0,100,200,300$, $400,500,600,700$, and $750 \mu \mathrm{m}$ from the center of the membrane (A point), respectively. The change in resonant frequency for the four resonant modes of the epitaxial PZT membrane as a function of the mass position along the radius was simulated, and the results are shown in figure 6.5. The change in frequency depends strongly on the mass position. For the 01- and 02-modes, the highest sensitivity is obtained at the center of the membrane $(\mathrm{x}=0 \mu \mathrm{m})$. The sensitivity of the 11 -mode is highest at the position $\mathrm{D}(\mathrm{x}=300 \mu \mathrm{m})$, while the highest sensitivity of the 21-mode is found at the position $\mathrm{E}(\mathrm{x}=400$ $\mu \mathrm{m})$. The modal shapes reveal that the membrane exhibits the highest sensitivity when the mass is positioned at the vibration antinode as shown in the insets of figure 6.5. These results show that it is 
necessary to use certain areas of the membrane for specific vibration mode to obtain the highest sensitivity.

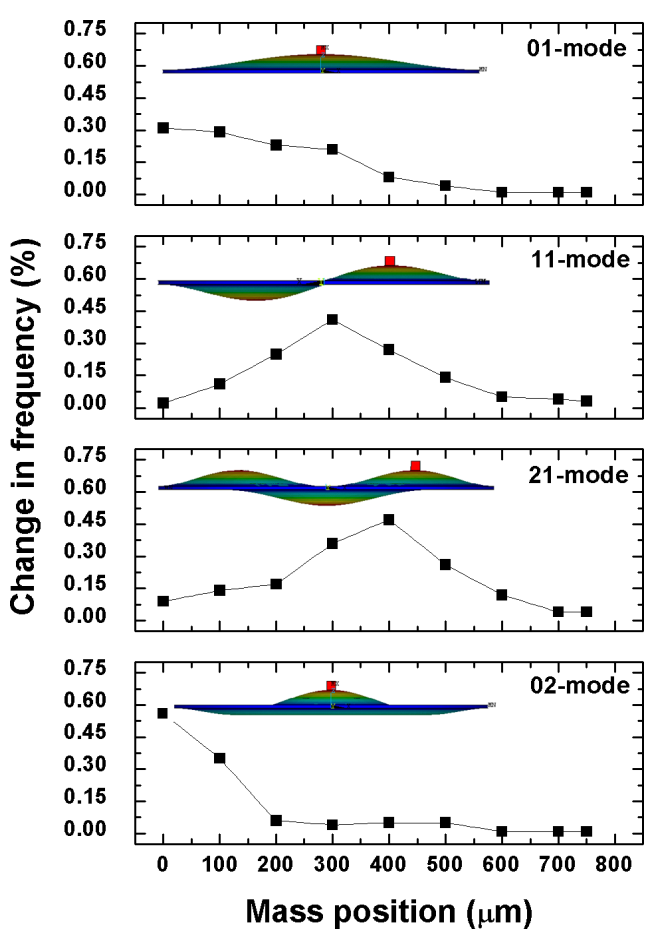

Figure 6.5. Relative change in resonance as a function of mass position for the four modes of vibration. The highest sensitivity found at positions $\mathrm{A}(\mathrm{x}=0 \mu \mathrm{m})$ for 01 -mode, $\mathrm{D}(\mathrm{x}=300 \mu \mathrm{m})$ for 11mode, $\mathrm{E}(\mathrm{x}=400 \mu \mathrm{m})$ for 21 -mode, and $\mathrm{A}(\mathrm{x}=0 \mu \mathrm{m})$ for 02 -mode. The insets show corresponding modal shapes of the epitaxial PZT membrane with the polystyrene box positioned at the antinode, where the highest sensitivity is obtained.

The sensitivity at the antinodal points for different vibration modes as a function of added mass is also investigated. The mass ranged from 15 to $300 \mathrm{ng}$ was placed on the antinode A for the 01- and 02-modes, and the antinodes E and D for the 11- and 21-modes, respectively. The response as a function of the added mass exhibits a linear behavior for all vibration modes as shown in figure 6.6(a). From the slopes of the linear fits, the mass sensitivity was calculated for all modes, and ranged from 260 to $45 \mathrm{pg} \mathrm{Hz}^{-1}$, depending on the vibration mode. The absolute change in frequency increases with the higher vibration mode. On the contrary, if the mass positions are at the nodes of vibrations (node $\mathrm{A}$ for the 11- and 21-modes and node $\mathrm{H}$ for the 01- and 02-modes), the change in frequency is significantly lower than placing mass at the antinode, and the response is unpredictable when varying the mass as shown in figure 6.6(b). 

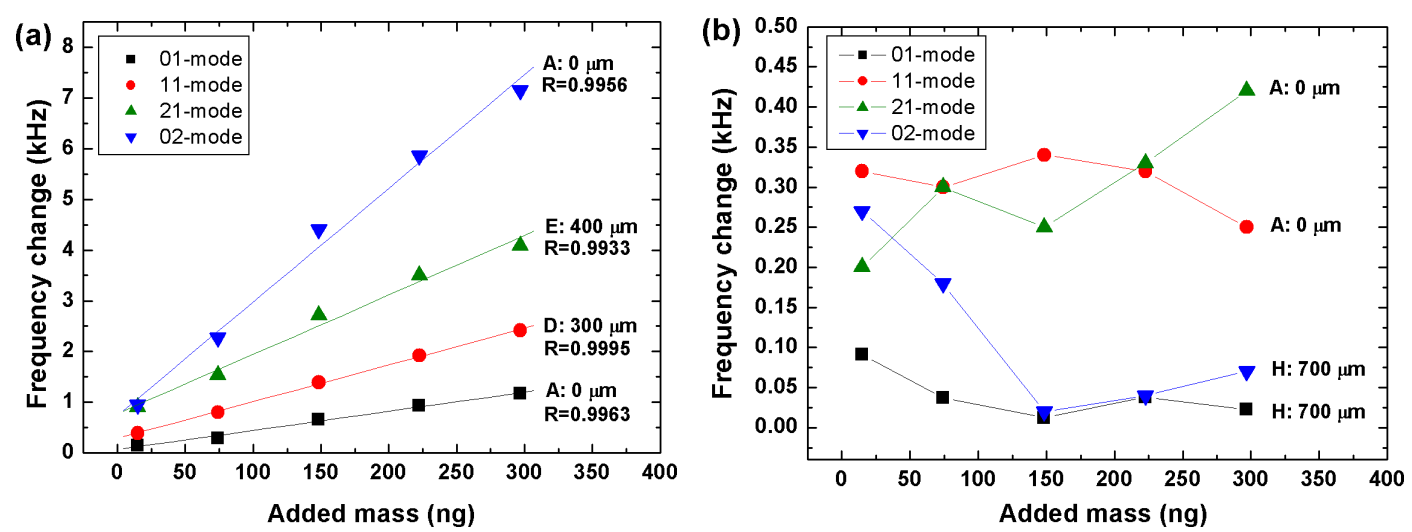

Figure 6.6. Change in frequency versus added mass at (a) antinode and (b) node positions.

Through the finite element analysis, we observe two important characteristics related to the sensitivity of the epitaxial PZT membrane. Firstly, the sensitivity is a strong function of the mass position, which is changing with the vibration mode. Specifically, the sensitivity is highest at the antinodal points. Secondly, the use of higher modes enhances the sensitivity of the membrane based mass sensor. This implies that the membrane sensor operating at higher modes can detect a smaller mass compared to the fundamental mode. As the results, the mass sensing areas must be located at the vibration antinodes of the membrane for optimum sensitivity. For symmetrical vibration modes, the antinodes are always at the center of the membrane, while the antinodes for asymmetrical modes vary depending on the modes of vibration. It is, however, difficult to examine the antinodes of asymmetrical modes in the sensor design phase. Thus, we propose to use only the symmetrical modes and define the mass position at the center of membranes. In the experiments, a focus is laid on the investigation of different capabilities in the localized-mass detection for an epitaxial membrane excited in symmetrical vibration modes. It should be noted that these analyses are not restricted to epitaxial PZT circular membranes, but they can be applied to a variety of membrane type sensors.

\subsection{Experimental results and discussion}

Several experiments have been carried out to evaluate operating characteristics of the epitaxial PZT membranes. The first experiment was conducted to examine the dynamic behavior of the epitaxial PZT membranes by presenting measurements on the resonant frequencies, modal shapes, and $Q$ factors. In this work, an optical technique based on laser vibrometry is used to investigate the dynamic behavior. This technique allows us to observe all modes of resonances and especially modal shapes, which is an advantage over an electrical impedance measurement [44, 45]. The mass loading and sensing characterizations were then performed to estimate the mass sensitivity. Finally, the resonant frequency fluctuation of the system was determined to find the minimum detectable mass. All 
experiments were performed at atmospheric pressure and at room temperature. The instrument setup was placed on a vibration-free table to avoid mechanical noises.

\subsubsection{Resonance characterization}

The resonant frequencies, modal shapes, and quality factors of the epitaxial PZT membranes are characterized using a Polytec MVS-400 laser Doppler vibrometer. The membranes are first excited by a white noise signal with an amplitude of $1 \mathrm{~V}$ in the frequency range of 50 to $400 \mathrm{kHz}$ to find the first four modes of resonances. The laser beam is positioned slightly off-centered from the center of the membrane in order to detect both symmetrical and asymmetrical modes of vibrations. The typical frequency response of a $1500 \mu \mathrm{m}$ wide membrane is presented in figure 6.7. In order to determine the right resonant frequencies, excluding the response peaks due to environmental inputs, the modal shapes of vibrations are characterized by means of laser scanning while applying a $1 \mathrm{~V}$ sinusoidal signal. The insets of figure 6.7 illustrate the corresponding modal shapes obtained using the laser scanning. The 01 -mode resonance appeared at $94.38 \mathrm{kHz}$, the 11 -mode at $192.96 \mathrm{kHz}$, the 21 -mode at $317.81 \mathrm{kHz}$, and the 02 -mode at $353.75 \mathrm{kHz}$. In table 6.2 , the simulated resonant frequencies are compared with the measured ones. The resonant frequency for the 01-mode resonance shows a small deviation from the simulated value. The deviation is larger for the higher vibration modes, which is probably due to the finite flexural rigidity of the structure [46]. These measured resonant frequencies, however, are in good agreement within a few percent with the simulated ones. The $Q$ factor of the membrane is also analyzed at atmospheric pressure, for the different modes of resonances. In this case, the membrane is actuated by a sinusoidal signal with the amplitude of $1 \mathrm{~V}$. The laser beam is positioned on the center of the membrane for detecting the symmetrical modes and on off-center for detecting the asymmetrical modes. The frequency responses for all four modes are shown in figure 6.8. The $Q$ factors are found to be $\sim 110$ for the 01 - and 11-modes, while increasing to be $\sim 144$ for the 21- and 02-modes. Remarkably, the membrane exhibits high $Q$ factors in atmospheric pressure even when actuated by a very thin epitaxial PZT film. 


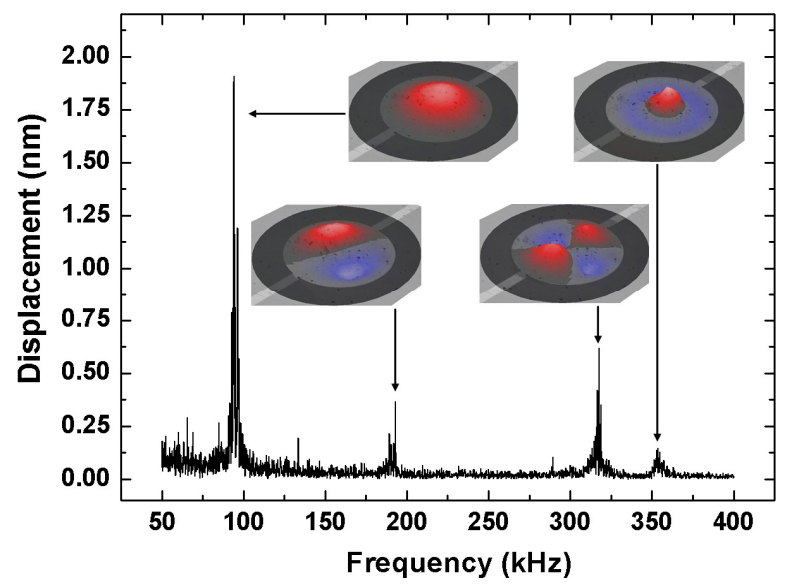

Figure 6.7. Typical frequency response of an epitaxial PZT membrane. The corresponding modal shapes are shown in the insets. The first four modes of resonances were found to be at 94.38, 192.96, 317.81 , and $353.75 \mathrm{kHz}$.

Table 6.2. Comparison of simulated and experimental values of resonant frequencies for different modes of operations.

\begin{tabular}{lcccc}
\hline Resonant mode & 01-mode & 11-mode & 21-mode & 02-mode \\
\hline Simulated values (kHz) & 94.11 & 196.23 & 322.79 & 367.43 \\
Experimental values (kHz) & 94.38 & 192.96 & 317.81 & 353.75 \\
Deviation (\%) & 0.28 & 1.67 & 1.54 & 3.72 \\
\hline
\end{tabular}

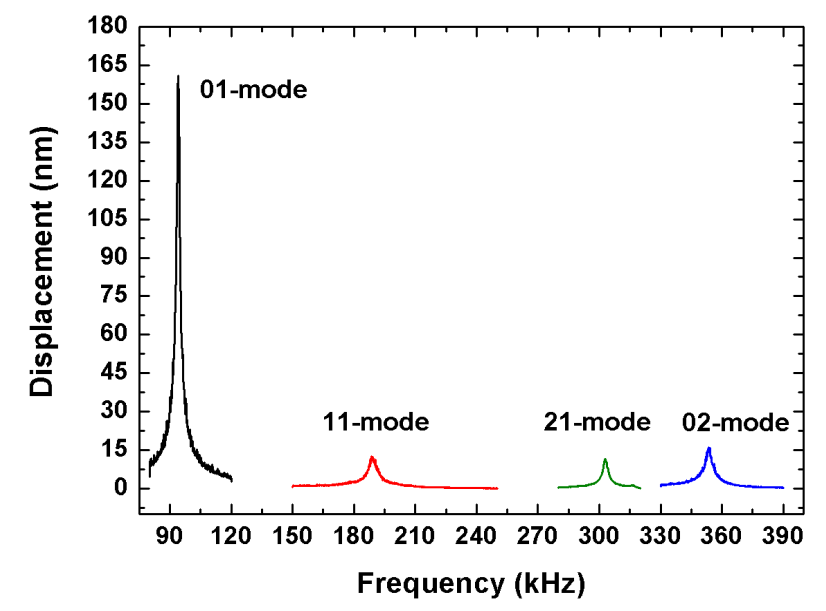

Figure 6.8. Resonant frequencies of an epitaxial piezoelectric membrane for four resonant modes actuated by a sinusoidal signal with the amplitude of $1 \mathrm{~V}$. 


\subsubsection{Mass loading and sensing}

Once the resonant frequencies of the epitaxial PZT membranes without the added mass have been characterized as the reference frequencies, the mass loading characterizations are carried out. The basic principle of mass sensors operating in resonant mode is the measurement of the resonant frequency shift due to the added mass to the sensor surface. For the estimation of sensor sensitivity, polystyrene microspheres (Polysciences, Inc.) with a diameter of $30 \mu \mathrm{m} \pm 0.22 \mu \mathrm{m}$ are used for the localized-mass loading on the epitaxial PZT membranes [47]. These microspheres are suspended in de-ionized water with residual surfactant. The polystyrene density is $\sim 1.05 \mathrm{~g} \mathrm{~cm}^{-3}$ giving an average mass of $14.84 \mathrm{ng}$. A droplet of a suspension containing $30-\mu \mathrm{m}$ polystyrene microspheres is placed onto the surface of membranes using a syringe, and dried under nitrogen flow. A probing station equipped with a tip is used for manipulating the polystyrene microspheres into desired position. The position and number of polystyrene microspheres attached on the surface are determined by an optical microscope. The frequency responses as a function of the mass loading are characterized using the Polytec MVS-400 laser Doppler vibrometer at atmospheric pressure.

The resonant frequencies of an epitaxial PZT membrane are measured before and after different mass loadings. The polystyrene microspheres are positioned at the center of the membrane. The added mass area is always less than $0.6 \%$ of the total electrode surface. The membrane is actuated by a sinusoidal signal with the amplitude of $1 \mathrm{~V}$ in the range from 90 to $97.5 \mathrm{kHz}$, near its 01 -mode resonance. The frequency spectra of 01-mode resonance shown in figure 6.9 reveal a significant frequency shift toward to lower frequencies with increasing the mass. This first result confirms the proper operation of the device and instrumental setup, thereby providing confidence to pursue the next experiments. By using the same technique, the frequency change as a function of the mass loading for the other resonant modes is also investigated. Each experiment is repeated at least three times and the data presented here are the mean value of the results obtained. Figure 6.10 shows the measured frequency changes as a function of the added mass for the symmetrical (01- and 02-modes) and the asymmetrical (11-mode) resonances. The frequencies of the symmetrical resonances shift down in linear proportion to the amount of the added mass. For the 02-mode resonance, the down shifting of the resonant frequency is higher as expected. The experimental mass sensitivity obtained from the slop of the linear fit is found to be $170 \mathrm{pg} \mathrm{Hz}^{-1}$ for the 01 -mode resonance and $46 \mathrm{pg} \mathrm{Hz}^{-1}$ for the 02 mode resonance. The large increase in sensitivity with the higher mode of resonance is mainly due to a decrease in the effective mass as defined in equation (6.1). The frequency change values obtained from the simulations and from the experiments are compared, which are in good agreement. The detailed mass sensing results of the 01- and 02-mode resonances are specified in table 6.3. Most of the measured frequency changes are higher than the simulated values. This discrepancy could be due to an underestimation of the polystyrene microsphere density or the deviation of the microsphere diameter, the differences between the model and the final geometry of the deposited layers and the silicon membrane, and the variations of the material properties. On the other hand, the resonant 
frequency response of the asymmetrical mode is almost constant because the mass positions are at the nodes of vibrations (figure 6.10), which corresponds to the simulations. This result is also confirmed by the modal shape of vibration, tested by using the laser scanning as shown in figure 6.11.

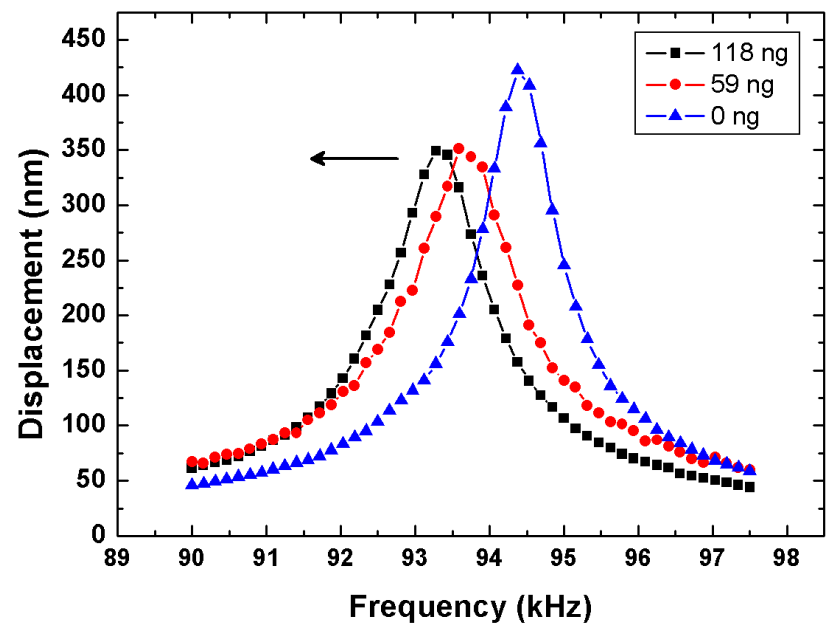

Figure 6.9. Typical frequency spectra of an epitaxial PZT membrane with different added mass.

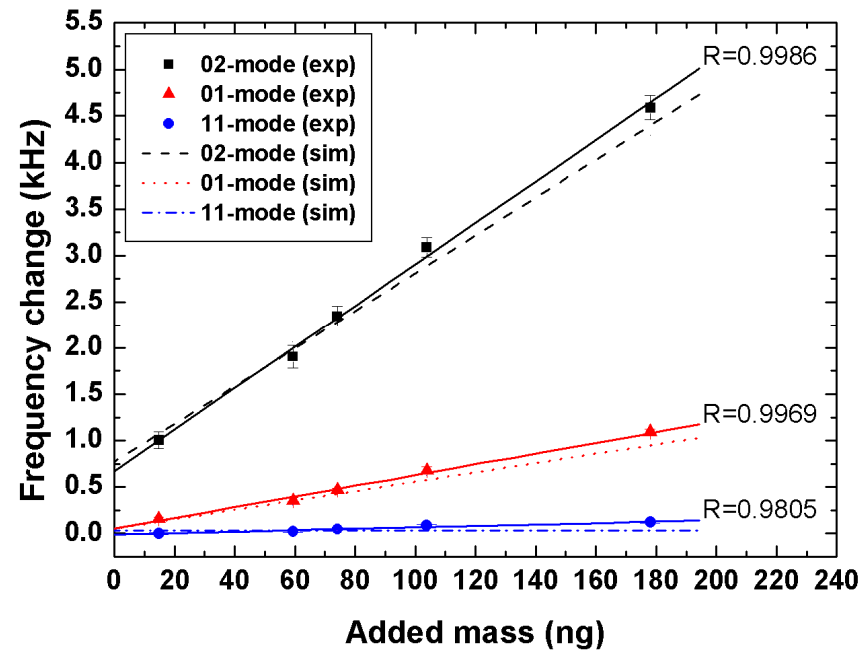

Figure 6.10. Measured frequency change as a function of added mass for symmetrical (01- and 02modes) and asymmetrical (11-mode) resonances compared to the simulations. The masses are positioned at the center of the membrane. The solid lines represent the linear fits to the measured data. Note that exp and sim represent experiment and simulation, respectively. 
Table 6.3. Change in frequency determined by simulation $\left(\Delta f_{s i m}\right)$ and experiment $\left(\Delta f_{\text {exp }}\right)$ for the symmetrical 01- and 02-modes of resonances.

\begin{tabular}{lcccccc}
\hline \multirow{2}{*}{ Added mass $(\mathrm{ng})$ Mode-01 } & \multicolumn{3}{c}{ Mode-02 } \\
\cline { 2 - 7 } & $\Delta f_{\text {sim }}(\mathrm{kHz})$ & $\Delta f_{\text {exp }}(\mathrm{kHz})$ & Deviation $(\%)$ & $\Delta f_{\text {sim }}(\mathrm{kHz})$ & $\Delta f_{\text {exp }}(\mathrm{kHz})$ & Deviation (\%) \\
\hline 14.84 & 0.1420 & 0.1575 & 10.92 & 0.9500 & 1.0052 & 5.81 \\
59.36 & 0.3034 & 0.3277 & 8.01 & 2.0665 & 1.9053 & 7.80 \\
74.20 & 0.4230 & 0.4688 & 10.83 & 2.2700 & 2.3450 & 3.30 \\
103.88 & 0.6273 & 0.6815 & 8.64 & 3.0438 & 3.0860 & 1.39 \\
178.08 & 0.9367 & 1.0938 & 16.77 & 4.2951 & 4.5890 & 6.84 \\
\hline
\end{tabular}

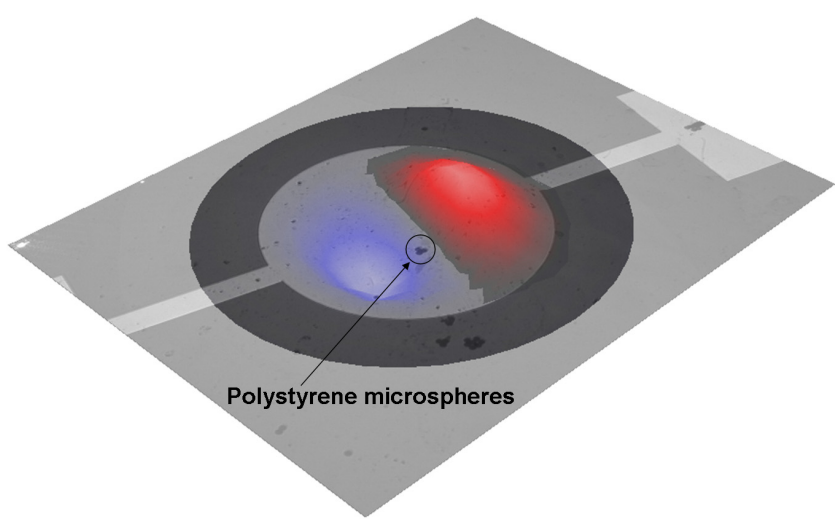

Figure 6.11. Modal shape of the 11-mode resonance of the epitaxial PZT membrane with the polystyrene microspheres at the vibration node.

The limit of detection for the current measurement setup and the epitaxial PZT membrane configuration is determined by the fluctuation level of resonant frequency together with the mass sensitivity. The resonant frequency data without any mass loading are recorded over $30 \mathrm{~min}$ using the laser Doppler vibrometer. The measured fluctuation level of the 01-mode resonance is shown in figure 6.12(a). The standard deviation of the resonant frequency variation is about $30 \mathrm{~Hz}$. Thus, the LOD is calculated using equation (6.2) to be about $5.1 \mathrm{ng}$. For the 02-mode resonance, the resonant frequency fluctuation of $107 \mathrm{~Hz}$ is observed as shown in figure 6.12(b) resulting in the LOD of $4.9 \mathrm{ng}$. Although, the 02-mode resonance gives a higher resonant frequency shift than the 01-mode resonance, the resonant frequency fluctuation obtained from 02 -mode resonance has a larger value than that obtained using the 01-mode resonance. The larger resonant frequency fluctuation of the $02-$ mode resonance is due to the frequency instability, which gives rise to the increased uncertainty in the peak position $[12,48]$. As the result, the minimum detectable mass of the 02-mode resonance is 
similar to that of the 01-mode resonance. The frequency stability of the sensor depends on different noise sources, e.g. thermomechanical noise, temperature fluctuations, and electrical noise [49]. By refining the measurement setup to reduce the noises, the resonant frequency fluctuation should be improved significantly to values as low as units of millihertz, and thus the minimum detectable mass would reach to the range of picograms.

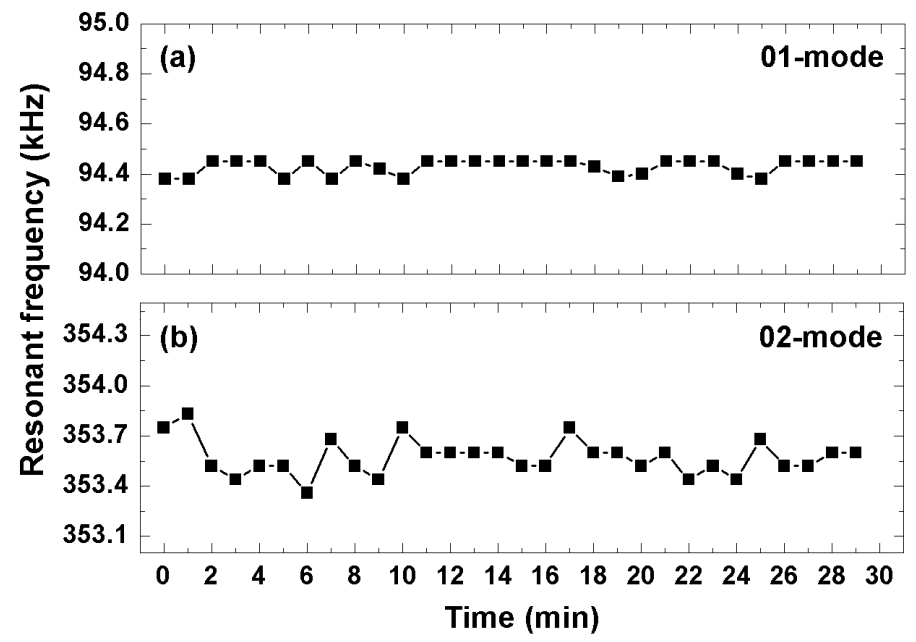

Figure 6.12. Resonant frequency fluctuations: (a) 01-mode and (b) 02-mode.

The experimental results presented here reveal the capabilities of circular silicon membranes actuated by the epitaxial PZT thin films as mass sensing devices. The silicon membrane actuated by a thin epitaxial PZT layer demonstrates a strong harmonic oscillation with a high $Q$ factor due to excellent piezoelectric properties of the epitaxial films. The experimental results show excellent agreement with the FEM analyses. For optimum sensitivity, the specific position of the mass loading must be defined for a given resonant mode. Based on our numerical and experimental results, we propose to specify the mass position at the center of membrane and use the symmetrical modes of vibrations. Currently, the mass sensitivity of the epitaxial PZT membrane as a localized-mass sensor is of the order $10^{-12} \mathrm{~g} \mathrm{~Hz}^{-1}$ at atmospheric pressure, which allows us to detect DNA molecules [10], proteins [48], cells [50], and mercury vapor [51]. For these different sensing applications, the epitaxial PZT membranes have to be coated locally with specific functional layers. One possible method is to use the drop-on-demand inkjet printing technique, which can handle various solution-based materials and deliver small amounts of liquids with good spatial accuracy [52]. Further enhancement of the mass sensing sensitivity can be achieved by reducing the size of a membrane. The finite element analysis predicts that the mass sensitivity will approach $10^{-15} \mathrm{~g} \mathrm{~Hz}^{-1}$, when the epitaxial PZT membrane is reduced to $500 \mu \mathrm{m}$ in diameter with the silicon thickness of $5 \mu \mathrm{m}$. With the current measurement setup, the minimum detectable mass is limited by the high noise level. With the use of 
an improved measurement setup, one can expect the reduction of the frequency fluctuation leading to a better minimum detectable mass value.

\subsection{Conclusions}

In this study, we presented a circular silicon membrane actuated by an epitaxial piezoelectric PZT thin film for mass sensing applications. The integration of epitaxial PZT thin film grown on silicon substrates with a metallic $\mathrm{SrRuO}_{3}$ bottom electrode, exhibits high crystalline quality and excellent piezoelectric properties. The epitaxial PZT membrane device was successfully fabricated through a sequence of microfabrication processes optimized for epitaxial oxide layers. A 150-nm thick epitaxial PZT film is able to actuate the $1500-\mu \mathrm{m}$ membrane with a strong harmonic oscillation response and a high $Q$ factor at atmospheric pressure, promising characteristics for mass sensing applications. The mass sensing performances of the proposed structure were evaluated both numerically and experimentally. It is found that the sensitivity is a function of the mass position, where is highest at the antinodal points. Therefore, we propose to use only the symmetrical modes of vibrations and define the mass position at the center of membranes as a localized-mass sensor for the optimum sensitivity and ease of sensor design. The epitaxial PZT membrane demonstrates a mass sensitivity of $170 \mathrm{pg} \mathrm{Hz}^{-1}$ for the 01 -mode resonance, and the sensitivity increases significantly to $46 \mathrm{pg} \mathrm{Hz}^{-1}$ for the 02 -mode resonance. The experimentally observed changes in resonant frequency are in excellent agreement with the numerical analyses. This sensor with the mass sensitivity of the order $10^{-12} \mathrm{~g} \mathrm{~Hz}^{-1}$ and the minimum detectable mass of $5 \mathrm{ng}$ has the potential for the detection of small quantities of biomaterials. Further improvements on measurement setups and optimization of the sensor dimensions will allow enhancing the quality factor, resulting in higher mass sensitivity, smaller minimum detectable mass, and better signal-to-noise ratio since $1 / Q$ represents the intrinsic noise of a system [53]. Future study will address these issues as well as the sensing performance of the epitaxial PZT membrane in a viscous environment.

\section{Acknowledgements}

This work was supported by the Swiss National Science Foundation through the National Centre of Competence in Research 'Materials with Novel Electronic Properties' MaNEP and by the European Union through the project 'Nanoxide'. The work at Yale was supported by NSF MRSEC DMR 0520495 and the FENA center. Don Isarakorn is grateful to the Ministry of Science and Technology, Thailand for the financial support for his PhD studies at EPFL IMT-NE SAMLAB. 


\section{References}

[1] Xu T, Wang Z, Miao J, Yu L and Li C M 2008 Micro-machined piezoelectric membranebased immunosensor array Biosens. Bioelectron. 24 638-643

[2] Tsai J Z, Chen C J, Chen W Y, Liu J T, Liao C Y and Hsin Y M 2009 A new PZT piezoelectric sensor for gravimetric applications using the resonance-frequency detection Sens. Actuators, B 139 259-264

[3] Pang W, Yan L, Zhang H, Yu H, Kim E S and Tang W C 2006 Femtogram mass sensing platform based on lateral extensional mode piezoelectric resonator Appl. Phys. Lett. 88 243503

[4] Gonzalez-Castilla S, Olivares J, Clement M, Iborra E, Sangrador J, Malo J and Izpura J I 2008 Electrical detection of the mechanical resonances in AlN-actuated microbridges for mass sensing applications Appl. Phys. Lett. 92183506

[5] Kwon T Y, Eom K, Park J H, Yoon D S, Kim T S and Lee H L 2007 In situ real-time monitoring of biomolecular interactions based on resonating microcantilevers immersed in a viscous fluid Appl. Phys. Lett. 90223903

[6] Yang Y T, Callegari C, Feng X L, Ekinci K L and Roukes M L 2006 Zeptogram-scale nanomechanical mass sensing Nano Lett. 6 583-586

[7] Ilic B, Czaplewski D, Zalalutdinov M, Craighead H G, Neuzil P, Campagnolo C and Batt C 2001 Single cell detection with micro mechanical oscillators J. Vac. Sci. Technol. B 192825 2828

[8] Shin S, Lee N -E, Park H -D, Park J -S and Lee J 2006 Piezoelectrically driven microtransducer mass sensors Integr. Ferroelectr. 80 355-362

[9] Carlen E T, Weinberg M S, Dube C E, Zapata A M and Borenstein J T 2006 Micromachined silicon plates for sensing molecular interactions Appl. Phys. Lett. 89173123

[10] Nicu L, Guirardel M, Chambosse F, Rougerie P, Hinh S, Trevisiol E, Francois J -M, Majoral J -P, Caminade A -M, Cattan E and Bergaud C 2005 Resonating piezoelectric membranes for microelectromechanically based bioassay: detection of streptavidin-gold nanoparticles interaction with biotinylated DNA Sens. Actuators, B 110 125-136

[11] Jeon Y, Wong C W and Kim S -G 2004 Water-immersible micromachined $\mathrm{Pb}(\mathrm{Zr}, \mathrm{Ti}) \mathrm{O}_{3}$ thin film actuators $J$. Electroceram. 13 509-513

[12] Ayela C and Nicu L 2007 Micromachined piezoelectric membranes with high nominal quality factors in Newtonian liquid media: A Lamb's model validation at the microscale Sens. Actuators, B 123 860-868

[13] Alava T, Mathieu F, Mazenq L, Soyer C, Remiens D and Nicu L 2010 Silicon-based micromembranes with piezoelectric actuation and piezoresistive detection for sensing purposes in liquid media J. Micromech. Microeng. 20075014 
[14] Ayela C, Alava T, Lagrange D, Remiens D, Soyer C, Ondarcuhu T, Greve A and Nicu L 2008 Electronic scheme for multiplexed dynamic behavior excitation and detection of piezoelectric silicon-based micromembranes IEEE Sens. J. 8 210-217

[15] Sokmen U, Stranz A, Waag A, Ababneh A, Seidel H, Schmid U and Peiner E 2010 Evaluation of resonating Si cantilvers sputter-deposited with AIN piezoelectric thin films for mass sensing applications J. Micromech. Microeng. 20064007

[16] Park J H, Kwon T Y, Yoon D S, Kim H and Kim T S 2005 Fabrication of microcantilever sensors actuated by piezoelectric $\mathrm{Pb}\left(\mathrm{Zr}_{0.52} \mathrm{Ti}_{0.48}\right) \mathrm{O}_{3}$ thick films and determination of their electromechanical characteristics Adv. Funct. Mater. 15 2021-2028

[17] Defay E, Le Rhun G, Perruchot F, Rey P, Suhm A, Aid M, Liu L J, Pacheco S and Miller M 2009 Piezoelectric PZT thin films in the $100 \mathrm{~nm}$ range: a solution for actuators embedded in low voltage devices Proceedings of the $22^{\text {nd }}$ IEEE International Conference on Micro Electro Mechanical Systems (MEMS 2009) (Sorrento, Italy) pp 619-622

[18] Reilly E K and Wright P K 2009 Modeling, fabrication and stress compensation of an epitaxial thin film piezoelectric microscale energy scavenging device J. Micromech. Microeng. 19095014

[19] Ramesh R and Schlom D G 2002 Orienting Ferroelectric Films Science 296 1975-1976

[20] Isarakorn D, Sambri A, Janphuang P, Briand D, Gariglio S, Triscone J -M, Guy F, Reiner J W, Ahn C H, de Rooij N F 2010 Epitaxial piezoelectric MEMS on silicon J. Micromech. Microeng. 20055008

[21] Ekinci K L, Yang Y T and Roukes M L 2004 Ultimate limits to inertial mass sensing based upon nanoelectromechanical systems J. Appl. Phys. 61 2682-2689

[22] Lochon F, Dufour I and Rebiere D 2005 An alternative solution to improve sensitivity of resonant microcantilever chemical sensors: comparison between using high-order modes and reducing dimensions Sens. Actuator, B 108 979-985

[23] Campanella H, Esteve J, Montserrat J, Uranga A, Abadal G, Barniol N and RomanoRodriguez A 2006 Localized and distributed mass detectors with high sensitivity based on thin-film bulk acoustic resonators Appl. Phys. Lett. 89033507

[24] Verd J, Uranga A, Abadal G, Teva J, Torres F, Perez-Murano F, Fraxedas J, Esteve J and Barniol N 2007 Monolithic mass sensor fabricated using a conventional technology with attogram resolution in air conditions Appl. Phys. Lett. 91013501

[25] Xin Y, Li Z, Odum L, Cheng Z - Y and Xu Z 2006 Piezoelectric diaphragm as a high performance biosensor platform Appl. Phys. Lett. 89223508

[26] Jin D, Li X, Bao H, Zhang Z, Wang Y, Yu H and Zuo G 2005 Integrated cantilever sensors with a torsional resonance mode for ultraresoluble on-the-spot bio/chemical detection Appl. Phys. Lett. 90041901 
[27] Tamayo J 2005 Study of the noise of micromechanical oscillators under quality factor enhancement via driving force control J. Appl. Phys. 97044903.

[28] Reiner J W, Garrity K F, Walker F J, Ismail-Beigi S and Ahn C H 2008 Role of strontium in oxide epitaxy on silicon (001) Phys. Rev. Lett. 101105503

[29] Su D, Yang B, Jiang N, Sawicki M, Broadbridge C, Couillard M, Reiner J W, Walker F J, Ahn C H and Zhu Y 2010 Valence electron energy-loss spectroscopy of ultrathin $\mathrm{SrTiO}_{3}$ films grown on silicon (100) single crystal Appl. Phys. Letter. 96121914

[30] Gariglio S, Stucki N, Triscone J -M and Triscone G 2007 Strain relaxation and critical temperature in epitaxial ferroelectric $\mathrm{Pb}\left(\mathrm{Zr}_{0.20} \mathrm{Ti}_{0.80}\right) \mathrm{O}_{3}$ thin films Appl. Phys. Lett. 90202905

[31] Smits J and Choi W 1991 The constituent equations of piezoelectric heterogeneous bimorphs IEEE Trans. Ultrason. Ferroelectr. Freq. Control 38 256-270

[32] Hishikazu Y, Yang E -H, Cheng J -G and Trolier-McKinstry S 2004 Optimized design, fabrication and characterization of PZT unimorph microactuators for deformable mirrors Proceedings of 2004 ASME International Mechanical Engineering Congress and RD\&D Expo (California, USA) pp 299-304

[33] Wang C, Wang Z, Ren T -L, Zhu Y, Yang Y, Wu X, Wang H, Fang H and Liu L 2007 A micromachined piezoelectric ultrasonic transducer operating in $d_{33}$ mode using square interdigital electrodes IEEE Sens. J. 7 967-976

[34] Defay E, Millon C, Malhaire C and Barbier D 2002 PZT thin films integration for the realization of a high sensitivity pressure microsensor based on a vibrating membrane Sens. Actuators, A 99 64-67

[35] Dean J, Gibbs M R J and Schrefl T 2006 Finite-element analysis on cantilever beams coated with magnetostrictive material IEEE Trans. Magn. 42 283-288

[36] Watts R, Gibbs M R J, Karl W J and Szymczak H 1997 Finite-element modeling of magnetostrictive bending of a coated cantilever Appl. Phys. Lett. 70 2607-2609

[37] Fabian J -H, Scandella L, Fuhrmann H, Berger R, Mezzacasa T, Musil Ch, Gobrecht J and Meyer E 2000 Finite element calculations and fabrication of cantilever sensors for nanoscale detection Ultramicroscopy 82 69-77

[38] Luginbuhl Ph, Racine G -A, Lerch Ph, Romanowicz B, Brooks K G, de Rooij N F, Renaud Ph and Setter N 1996 Piezoelectric cantilever beams actuated by PZT sol-gel thin film Sens. Actuators, A 54 530-535

[39] 1990 Landolt-Börnstein: Numerical Data and Functional Relationships in Science and Technology New serie, Group III, vol 26 (Berlin: Springer-Verlag) p 123

[40] Pamula V K, Jog A and Fair R B 2001 Mechanical property measurement of thin-film gold using thermally actuated bimetallic cantilever beams Nanotech 1 410-413 
[41] Dohn S, Sandberg R, Svendsen W and Boisen A 2005 Enhance functionality of cantilever based mass sensors using higher modes Appl. Phys. Lett. 86233501

[42] Campanella H, Esteve J and Montserrat J 2006 Localized and distributed mass detectors with high sensitivity based on thin-film bulk acoustic resonators Appl. Phys. Lett. 89033507

[43] Ramos D, Tamayo J, Mertens J and Calleja M 2006 Origin of the response of nanomechanical resonators to bacteria adsorption Appl. Phys. Lett. 100106105

[44] Vazquez J, Sanz P and Sanchez-Rojas J L 2007 Behaviour of forbidden modes in the impedance characterization and modeling of piezoelectric microcantilevers Sens. Actuators, A $136417-425$

[45] Sanz P, Hernando J, Vazquez J and Sanchez-Rojas J L 2007 Laser vibrometry and impedance characterization of piezoelectric microcantilevers J. Micromech. Microeng. 17 931-937

[46] Hong E, Trolier-McKinstry S, Smith R, Krishnaswamy S V and Freidhoff C B 2006 Vibration of micromachined circular piezoelectric diaphragms IEEE Trans. Ultrason. Ferroelectr. Freq. Control 53 697-706

[47] Narducci M, Figueras E, Lopez M J, Gracia I, Santander J, Ivanov P, Fonseca L and Cane C 2009 Sensitivity improvement of a microcantilever based mass sensor Microelectron. Eng. 86 $1187-1189$

[48] Yi J W, Shih W Y and Shih W -H 2002 Effect of length, width, and mode on the mass detection sensitivity of piezoelectric unimorph cantilevers J. Appl. Phys. 91 1680-1686

[49] Lee J E -Y, Bahreyni B, Zhu Y and Seshia A A 2007 Ultrasensitive mass balance based on a bulk acoustic mode single-crystal silicon resonator Appl. Phys. Lett. 91234103.

[50] Ilic B, Czaplewski D, Craighead H G, Neuzil P, Campagnolo C and Batt C 2000 Mechanical resonant immunospecific biological detector Appl. Phys. Lett. 77 450-452

[51] Thundat T, Wachter E A, Sharp S L and Warmack R J 1995 Detection of mercury vapor using resonating microcantilevers Appl. Phys. Lett. 66 1695-1697

[52] Bietsch A, Zhang J, Hegner M, Lang H P and Gerber C 2004 Rapid functionalization of cantilever array sensors by inkjet printing Nanotechnology 15 873-880

[53] Cleland A N and Roukes M L 2002 Noise processes in nanomechanical resonators J. Appl. Phys. 92 2758-2769 



\title{
Chapter 7
}

\section{Evaluation of static measurement in piezoelectric cantilever sensors using a charge integration technique for chemical and biological detection}

\author{
D. Isarakorn, M. Linder ${ }^{\dagger}$, D. Briand and N. F. de Rooij \\ École Polytechnique Fédérale de Lausanne (EPFL), Institute of Microengineering (IMT), \\ The Sensors, Actuators and Microsystems Laboratory (SAMLAB), \\ Rue Jaquet-Droz 1, 2000 Neuchâtel, Switzerland \\ ${ }^{\dagger}$ Present address: picoDRILL SA, Lausanne, Switzerland
}

\begin{abstract}
In this paper, we introduce a charge integrator as a read-out system for piezoelectric cantilever sensors. The charge integrator is used to monitor the surface charge induced by piezoelectric materials as a function of mechanical stress. This technique improves the response of piezoelectric cantilever sensors in low frequency measurements which makes them suitable for chemical and biological detection. Furthermore, it is capable of real-time, portable and all-electrical detection. The detection of alkanethiols on the Au-coated piezoelectric cantilevers is demonstrated to verify the concept.
\end{abstract}




\subsection{Introduction}

There has been a growing interest in piezoelectric materials applied in micro-devices [1-6]. The piezoelectric cantilever sensor is a technology that has attracted much attention due to its potential as a sensing platform for chemical and biological detection. The piezoelectric cantilever offers many advantages in bio-sensing applications including high sensitivity, portability, and simplified sensing system. Most of the piezoelectric cantilevers are operated in dynamic mode, where the adsorption of biological molecules causes a shift in the resonance frequency [7-11]. However, they may not be suitable for the operation in viscous liquid since the sensitivity decreases dramatically due to low quality factor [12-14]. To overcome this problem, many chemical and biological sensors have been designed using the static detection method, where the surface stress produced on a cantilever can be observed as changes in cantilever deflection. Using this concept, the feasibility of chemical and biological detection with extremely high sensitivity has been demonstrated [15-21]. However, these sensors are based on silicon requiring bulky and expensive optical beam deflection measurements. The use of piezoelectric cantilevers in the static mode which is based upon changes in the deflection induced by the biomolecular interactions on the cantilever surface would be great promises for the detection of biological molecules in a liquid environment since they do not require bulky optical detection system. Nevertheless, when a quasi-static force is applied to the piezoelectric cantilever, the electrical charges developed by piezoelectric materials decay with a finite time constant. Utilizing a charge integrator, the charge decay on the piezoelectric cantilevers can be monitored. Hence, the detection of surface stress induced deflections in the piezoelectric cantilevers becomes possible. This measurement technique is not limited to only chemical and biological detection: it can be applied to a variety of piezoelectric sensors, such as impact sensors, piezo switches, fluid level sensors, and flow sensors.

In this paper, we report on the development of the charge integration technique for surface stress detection of piezoelectric cantilever sensors operating in the static mode. The concept of the charge integration technique and the detail of apparatus used to realize the detection system are presented. Several control experiments are performed to test the instrumental setup and sensor responses induced by environments, which provide useful information about any interference to sensor responses. The proposed technique is demonstrated with the detection of alkanethiols using $\mathrm{Au}$-coated piezoelectric cantilevers in liquid environment.

\subsection{Charge integration technique}

The charge integration technique was originally introduced for piezoelectric force transducers, which improved the response of piezoelectric transducers in low frequency force measurements [22]. Later, it was extensively applied for the determination of the piezoelectric coefficient of piezoelectric thin films [23-27], low-level current sensor measurements [28], and pressure sensors [29]. To our best knowledge, the use of charge integrators for bio-sensing applications has not been reported yet. In this 
study, we explore the feasibility to detect electrically and statically the deflections of piezoelectric cantilevers in real time and to develop a readout method suitable for a portable device based on the charge integrators. Detection of surface stress induced deflections in piezoelectric cantilever sensors can be easily performed by the charge integrator based detection system incorporating with reset-andintegrate operation.

The charge integrator based detection system consists of three main parts: (1) the piezoelectric cantilever, (2) the precision switched integrator IVC102 [30], and (3) reset-and-integrate measurement, as illustrated in figure 7.1. Each of these parts is described in the following subsections.
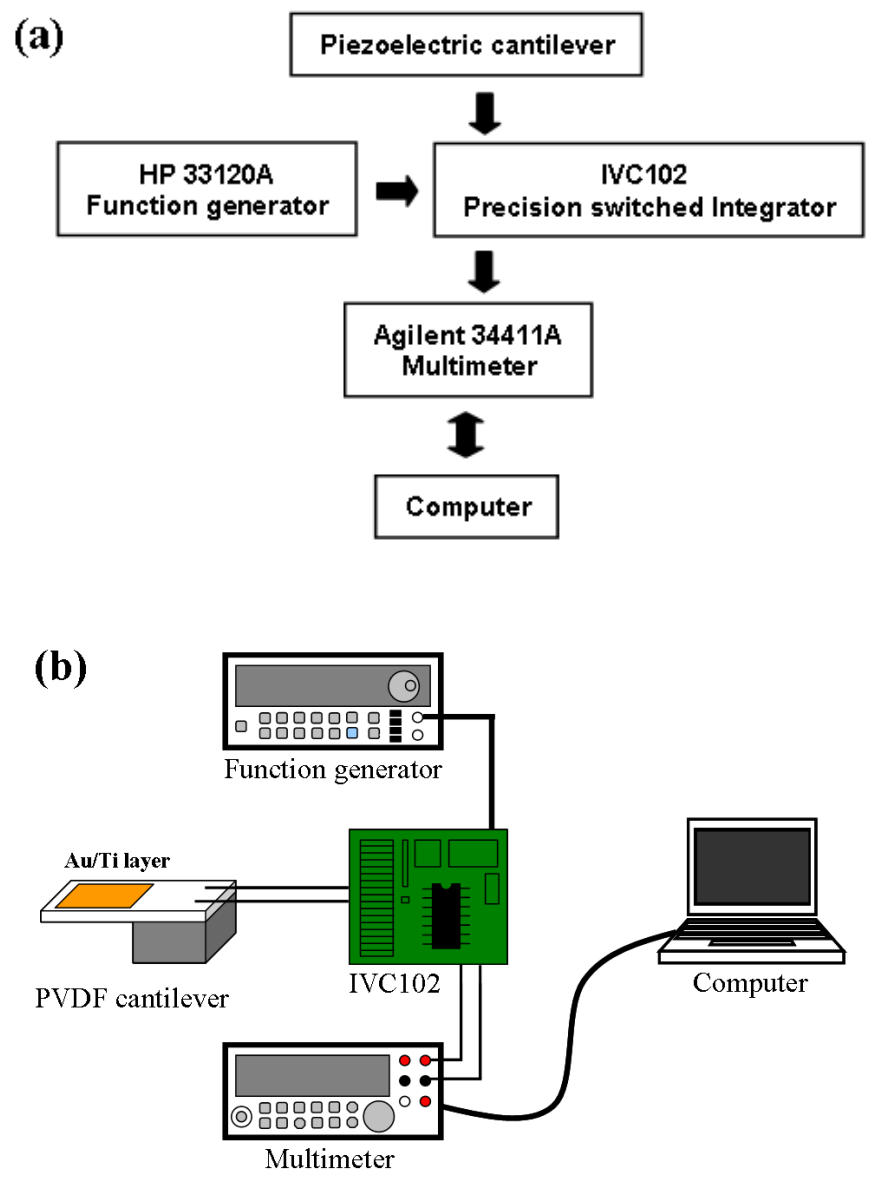

Figure 7.1. Charge integrator based detection system: (a) block diagram, (b) schematic of experimental setup.

\subsubsection{Piezoelectric cantilevers}

The surface stress induced by alkanethiol formation is monitored with an Au-coated Polyvinylidene Fluoride (PVDF) piezoelectric cantilever to demonstrate the concept of the charge integration technique. The PVDF cantilever is our choice due to excellent chemical stability and mechanical 
flexibility as well as commercially available at low cost $[29,31]$. Basically, the monolayer of alkanethiols is easily formed on the Au substrate due to the affinity of the sulfur atom to the $\mathrm{Au}$ surface. The alkanethiol molecule consists of three chemical entities: sulfur group, spacer group and terminal group. The formation of alkanethiol on gold is initiated by the strong chemical interactions between the sulfur and gold surface. The spacer group, which is typically a chain of methylene carbons, creates lateral interchain interactions to align the molecules parallel on the gold surface to form the self-assembled monolayers. The properties of the terminal group define the surface properties, which can be any desired functionality. In this work, the PVDF piezoelectric cantilevers LDT1-028K/L from Measurement Specialties Inc were used. The thickness of PVDF element is 28 $\mu \mathrm{m}$, which is laminated by $125-\mu \mathrm{m}$-thick polyester layer. The width and length are $16.25 \mathrm{~mm}$ and 32 $\mathrm{mm}$, respectively. The properties of the PVDF material taken from the company are listed in table 7.1. On the topside of the cantilever, a $\mathrm{Ti}(20 \mathrm{~nm})$ adhesion layer followed by an Au (100 nm) layer is deposited by e-beam evaporation as the sensitive layer for the alkanethiol formation.

Table 7.1. Main properties of PVDF material.

\begin{tabular}{lc}
\hline Property & Value \\
\hline Piezoelectric coefficient $d_{31}(\mathrm{pm} / \mathrm{V})$ & 23 \\
Electromechanical coupling $k_{31}$ & 0.12 \\
Dielectric constant & $12-13$ \\
Capacitance $(\mathrm{nF})$ & 1.38 \\
Young's modulus (Gpa) & $2-4$ \\
Maximum temperature $\left({ }^{\circ} \mathrm{C}\right)$ & 80 \\
\hline
\end{tabular}

\subsubsection{Precision switched integrator}

The change in the electrical charge due to surface stress of piezoelectric cantilevers can be detected using a charge integrator based on the precision switched integrator IVC102. The IVC102 is a precision integrating amplifier with FET op amp, integrating capacitors, and low leakage FET switches. The main function of this device is for measuring low-level sensor currents. Even fast current pulses are accurately measurable. The IVC102 can convert an input current or charge to an output voltage by integration. The circuit connection of the IVC102 with the piezoelectric cantilever for reset-and-integrate operation is shown in figure 7.2. The piezoelectric cantilever is connected to the inverting input of the IVC102, pin 2 . The switch $\mathrm{S}_{1}$ is always closed by connecting pin 11 to ground $(0 \mathrm{~V})$. During integration period, the input current $\left(I_{\text {in }}\right)$ is collected on the internal reference capacitors $C_{1}, C_{2}$ and $C_{3}$, which are connected in parallel for a total capacitance $\left(C_{t o t}\right)$ value of 100 
$\mathrm{pF}$. The switch $\mathrm{S}_{2}$ is used to control the integration and reset periods. A measurement cycle starts when $S_{2}$ opens and stops when $S_{2}$ closes. The output (pin 10) from the IVC102 is displayed as a voltage $\left(V_{o}\right)$ which is proportional to the amount of input charge collected on the internal capacitors.

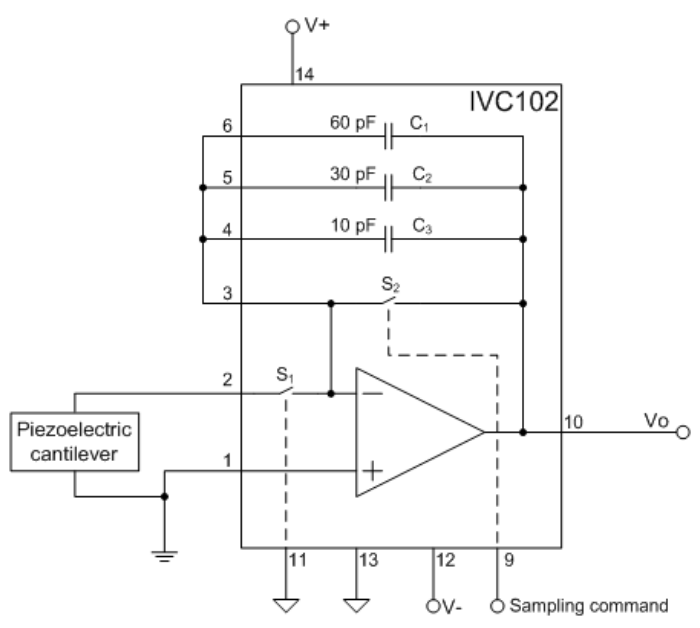

Figure 7.2. Schematic of precision switched integrator IVC102 connected to a piezoelectric cantilever.

\subsubsection{Reset-and-integrate operation}

The timing diagram of the reset-and-integrate operation is illustrated in figure 7.3. The surface stress induced charge from the PVDF piezoelectric cantilevers (figure 7.3(a)) is directed to a charge integrator based on the precision switched integrator IVC102. It integrates and stores the input current $\left(I_{\text {in }}\right)$ on the internal reference capacitors for a pre-determined period. The sampling command pulse (figure 7.3(b)) based on HP 33120A function generator is sent to IVC102 to control the sampling period $(T)$. The reset time must be longer than $10 \mu$ s to allow the op amp to slew to $0 \mathrm{~V}$. The range of integration period can range from $100 \mu$ s to many minutes depending on the total capacitance value and the input current. The integrate output voltage (figure 7.3(c)) depends on the input current from the piezoelectric cantilever and the period of integration time, which is defined as:

$$
V_{o}=-\int_{0}^{T} \frac{I_{\text {in }}}{C_{t o t}} d t
$$

The sampled output voltage (figure 7.3(d)) is then recorded in a computer-controlled Agilent 34411A multimeter. The surface stress profile (figure 7.3(e)) will be reconstructed by summing the sampled output voltage at the end of measurement. It should be noted that the accuracy of the measurements depends on the reset and integration period. These periods must be selected to meet the desired application requirements. 

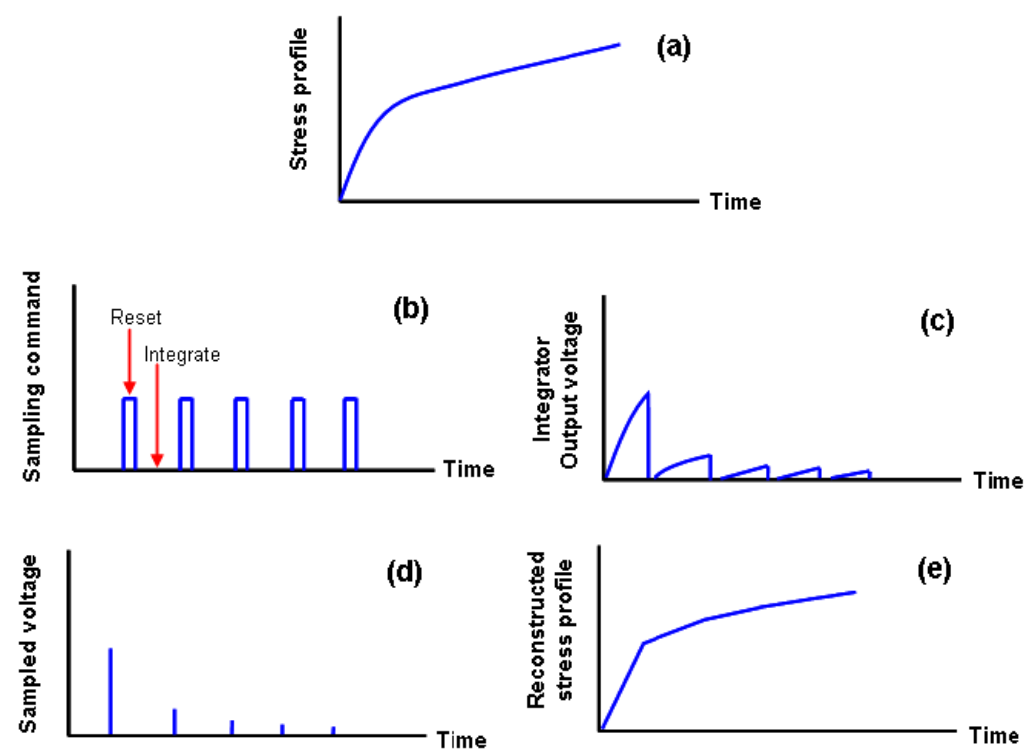

Figure 7.3. Timing diagram of reset-and-integrate operation.

\subsection{Results and discussion}

The use of PVDF cantilevers combined with the proposed charge integrator based detection system requires extensive testing of the instrumental setup and responses induced by environments. In this section, the first experiment is conducted to validate the operation of the IVC120 charge integrator circuit. The PVDF cantilevers are then expose to different environments to observe their responses. Finally, the detection of alkanethiols is demonstrated to verify the concept. All experiments presented here are carried out at room temperature (approx. $23^{\circ} \mathrm{C}$ ). The instrumental setup is placed on a vibration isolation table to avoid mechanical noises.

\subsubsection{Control experiments}

Prior to exposing the piezoelectric cantilevers to alkanethiol solutions, several experiments were performed to characterize the IVC102 charge integrator circuit and instrumental setup. The initial characterization experiment was conducted to test the output voltage of the IVC102 with different modes of operation. The PVDF cantilever LDT1-028K/L mounted on a PCB board was connected to the IVC102. The three modes of operation (Reset, Hold and Integrate) of the IVC102 are controlled by the $S_{1}$ and $S_{2}$ switches. Referring to figure 7.2, the modes of operation are summarized in table 7.2. For each mode of operation, an output voltage was recorded each $10 \mathrm{msec}$ over $300 \mathrm{sec}$ in the computer-controlled multimeter as shown in figure 7.4. 
Table 7.2. IVC102 modes of operation.

\begin{tabular}{|c|c|c|}
\hline \multirow{2}{*}{ Mode } & \multicolumn{2}{|c|}{ Switch } \\
\hline & $\mathbf{S}_{1}$ & $\mathbf{S}_{2}$ \\
\hline Reset & open & close \\
\hline Hold & open & open \\
\hline Integrate & close & open \\
\hline
\end{tabular}

In Reset mode, the integration capacitor is discharged and the output voltage is driven to $0 \mathrm{~V}$. The voltage response is stable over time. Then, Hold mode begins when $\mathrm{S}_{2}$ is opened. The output voltage should remain at the present voltage value at $0 \mathrm{~V}$. However, the offset voltage of $-18 \mathrm{mV}$ is observed. This offset error is due to the charge injection of $S_{2}$ opening. Moreover, a slow change in output voltage (Hold-mode droop) caused by op amp input bias current is also noticed. The droop rate of the IVC102 is typically $1 \mathrm{nV} \mu \mathrm{s}^{-1}$ [30], which rarely contributes significant error. For testing the Integrate mode, the sequence begins with opening $S_{1}$ and closing $S_{2}$ to reset the IVC102 to $0 \mathrm{~V}$ for 10 sec. Opening $\mathrm{S}_{2}$ (Hold mode) is then followed for another $10 \mathrm{sec}$ prior to integration. The offset voltage dominated by the charge injection of $S_{2}$ is around $-20 \mathrm{mV}$, which is nearly identical to the voltage response in the Hold mode test. The Integrate mode begins when $\mathrm{S}_{1}$ is closed. The residual charge in the PVDF cantilever transfers immediately to the internal capacitors resulting in a step output voltage.

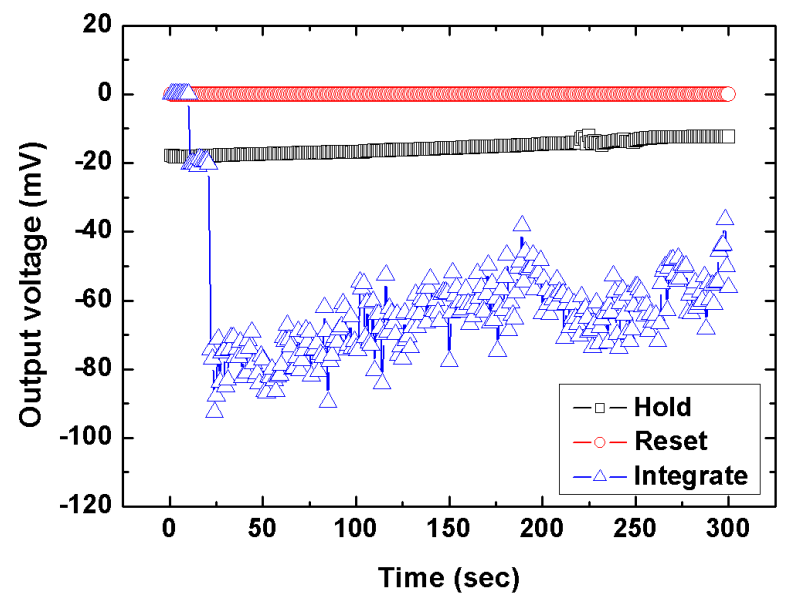

Figure 7.4. Typical output voltage curves of the IVC102 charge integrator circuit. 
The next experiment was conducted to confirm that the IVC102 was working correctly. We applied a force to the PVDF cantilever LDT1-028K/L by placing a $10 \mathrm{~g}$ mass of aluminum $\mathrm{M}_{1}$ onto the tip of the piezoelectric cantilever within the area of $10 \times 10 \mathrm{~mm}^{2}$. The stress induced deflection in the piezoelectric cantilever generated the current flowing into the internal reference capacitors, which produced the negative output voltage at $V_{\mathrm{o}}$ as shown in figure 7.5. When the mass $\mathrm{M}_{1}$ was applied to the PVDF cantilever, the output voltage quickly reached around $-6 \mathrm{~V}$ before stabilization around $-4 \mathrm{~V}$. This is due to the impact force upon applying the mass. At $25 \mathrm{sec}$, another $12 \mathrm{~g}$ mass of aluminum $\mathrm{M}_{2}$ was gently placed to avoid the impact force at the same position of $\mathrm{M}_{1}$, which induced more current charging into the internal capacitors. The integrator output voltage $V_{\mathrm{o}}$ from IVC102 responded as expected, which was proportional to the mass. It should be noted that the slow changes in output voltages after the application of the $\mathrm{M}_{1}$ and $\mathrm{M}_{2}$ are primarily due to small asymmetrical oscillations of the cantilever.

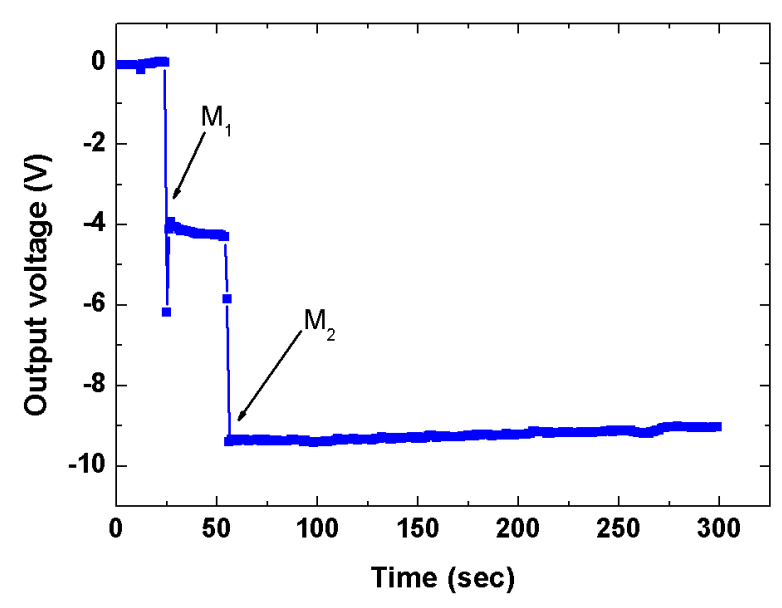

Figure 7.5. Integrator output voltage from mass loading.

For comparison between the voltage responses obtained with and without the charge integrator, a force was applied to the PVDF cantilever without the charge integrator by placing a $10 \mathrm{~g}$ mass. In this case, the cantilever was connected directly to the computer-controlled multimeter. Figure 7.6 shows the output voltage of the PVDF cantilever without the charge integrator. The voltage developed by the PVDF cantilever decay with a finite time constant. These results reveal the potential of the proposed technique to cope with the static measurement in piezoelectric sensors. 


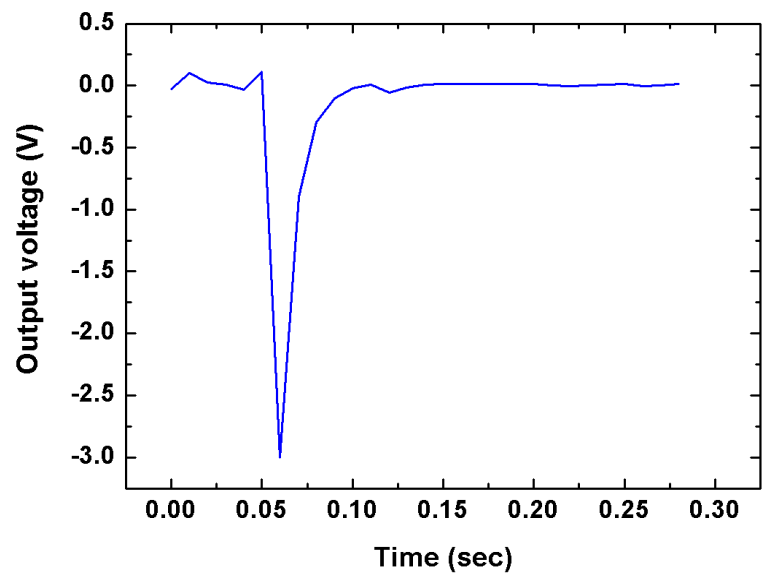

Figure 7.6. Output voltage of a PVDF cantilever without the IVC102 charge integrator circuit.

The piezoelectric cantilevers were then exposed to air and water for 8 hours in order to determine their responses to environments, which are not specifically induced by alkanethiols. There were no other factors involved in these experiments. The reset and integration period values are 0.5 sec and $1 \mathrm{sec}$, respectively. The output voltage in both cases was recorded each $100 \mathrm{msec}$ in the computer-controlled multimeter. We observed very small output voltage drifts of piezoelectric cantilevers both in air and water as shown in figure 7.7. The responses appear to be stable in both environments with minimal noise in the signal. These results provided important information about any interference with the integrator output voltage.

The results obtained from the control experiments confirm the function of the charge integrator readout and instrumental setup, thereby providing confidence to pursue the experiments of alkanethiol detections that will be demonstrated in the next section.
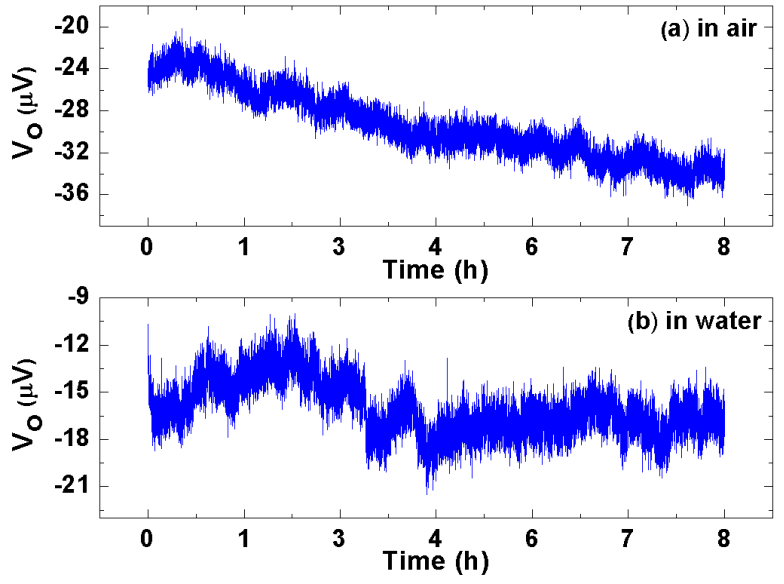

Figure 7.7. Output voltage drifts of piezoelectric cantilevers: (a) in air, (b) in water for 8 hours. 


\subsubsection{Alkanethiol detection}

Alkanethiols are known to form self-assembled monolayers (SAMs) on gold surfaces [17, 32-36]. There are two distinct phases involved in the alkanethiol monolayers [37]. The first phase is the adsorption of the sulfur groups on the Au surface. The adsorption depends strongly on thiol concentration. Higher concentration of thiol can lead to faster adsorption speed. This step is a very fast step, which usually takes only a few minutes. The second phase is a slow step (several hours) occurring gradually over time as the carbon chains orient themselves in an all-trans ordered fashion. The phenomena of surface stress (deflection) are investigated relative to the formation of alkanethiol SAMs on the Au-coated piezoelectric cantilevers to verify the effectiveness of the proposed detection system. In our experiments, two alkanethiols, 11-Mercaptoundecanoic (NanoThinks ${ }^{\mathrm{TM}}$ ACID11) and 16-Mercaptohexadecanoic (NanoThinks ${ }^{\mathrm{TM}}$ ACID16) acids purchased from Aldrich were used to study the self-assembly process on the gold surfaces of the piezoelectric cantilevers. These alkanethiols have the same head group (-SH) and terminal group (-COOH) but different length of alkyl chains. The concentrations of ACID11 and ACID16 in solution were held constant at $5 \mathrm{mM}$ in ethanol. All experiments were conducted at room temperature in the liquid phase. Before further processing, the piezoelectric cantilevers must be carefully cleaned. In this work, we cleaned the piezoelectric cantilevers in isopropanol and deionized water for 15 minutes each before gold deposition. The Ti/Au layer was then deposited onto the cleaned piezoelectric cantilevers. We again rinsed the Au-coated piezoelectric cantilevers with ethanol for 5 minutes just before the experiments.

The Au-coated piezoelectric cantilever was immerged vertically into ACID11 with the volume of $100 \mathrm{ml}$ in an enclosed glass container. A sample output voltage was recorded each 100 msec over 6 hours in the computer-controlled multimeter at the 0.5 -sec reset and 1-sec integration periods. The initial deflection was set to zero. Figure 7.8(a) shows the reconstructed sample output voltage due to the formation of ACID11 on the Au-coated piezoelectric cantilever. This result indicated the two phases involved in the formation of ACID11 monolayer [33]. The first phase was the initial adsorption of the sulfur groups on the Au surface, which took only minutes. The second phase was the organization of alkyl chains, where the deflection of piezoelectric cantilever was gradually increasing over time ( 5 hours). Once the SAM of alkanethiol has been formed completely, the response became stable. To check whether the voltage response was caused by the formation of ACID11 monolayer, a reference test was carried out on a PVDF cantilever without an Au layer. The voltage response labeled control displayed totally different from that obtained from the Au-coated piezoelectric cantilever, and was constant over time as shown in figure 7.8(b). These results revealed a significant change in surface stress due to the formation of ACID11 acid on the Au surface, confirming the effectiveness of the charge integrator based detection system. 

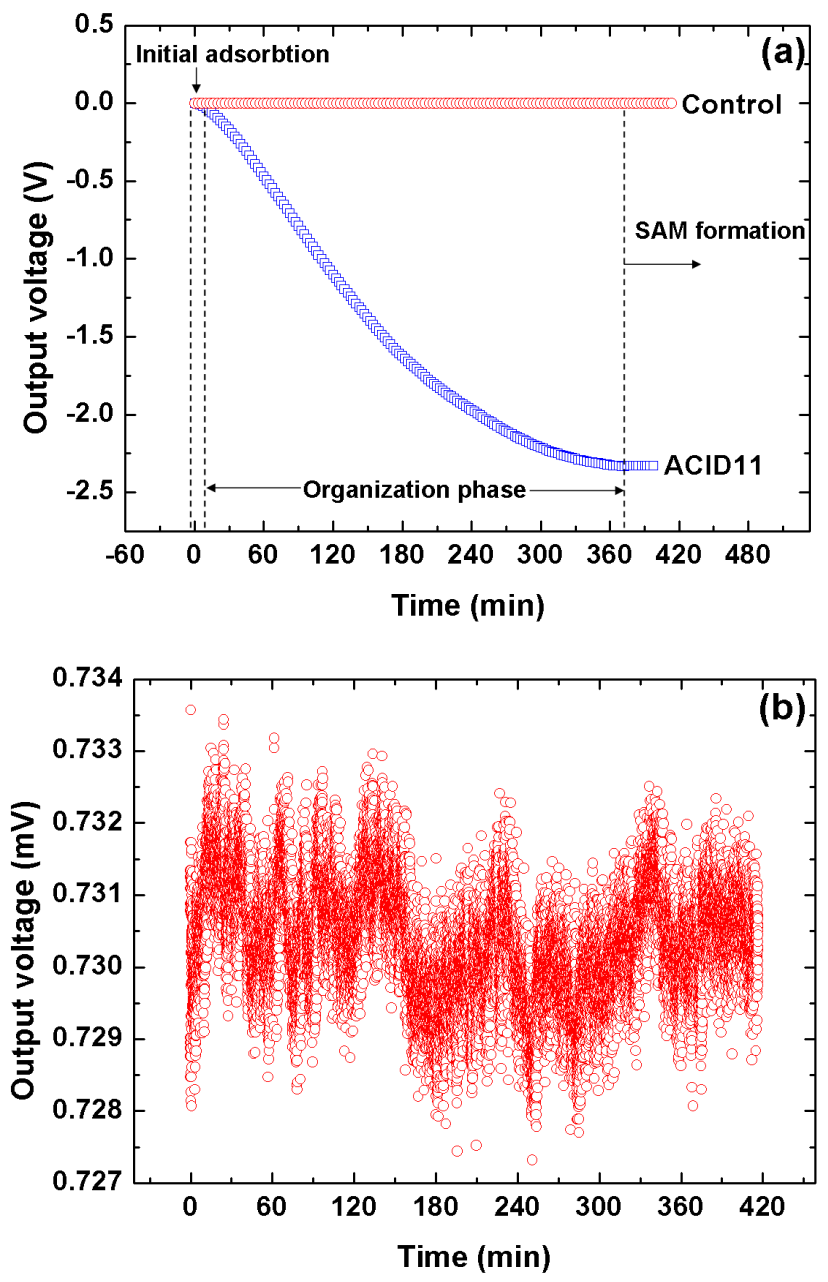

Figure 7.8. (a) Reconstructed surface stress profile due to the formation of ACID11 on the Au-coated piezoelectric cantilever obtained from the sampled output voltage. (b) The magnification of the voltage response labeled control of a PVDF cantilever without an Au layer.

To further investigate the detection of alkanethiol using the charge integration technique, we observed another alkanethiol having longer length of alkyl chains, ACID16. The experimental conditions were the same as those used with the ACID11. The result for the adsorption of ACID16 on the Au-coated piezoelectric cantilever compared with a PVDF cantilever without an Au layer is illustrated in figure 7.9. It is obvious that in the initial adsorption the ACID16 created a tensile stress on the surface resulting in the deflection of piezoelectric cantilever in upward direction. At approximately 20 minutes, the piezoelectric cantilever gradually began to bend in opposite direction (organization phase). The SAM formation of ACID16 seemed to be well ordered at approximately 4 hours, which was faster than that of ACID11 due to the Van der Waals interactions between the alkyl chains become stronger with the longer alkyl chain length promoting the production of SAM 
formation [38]. However, after 40 minutes of full monolayer formation, the piezoelectric cantilever bent in upward direction. It is believed that unbound alkanethaiol molecules formed the interplane hydrogen bonds on the terminal group $(-\mathrm{COOH})$ of alkanethiol monolayer leading to a second layer of alkanethiol on the top of SAM or unbound alkanethiols induced an imperfect SAM formation [39]. It was found that adding a small amount of $\mathrm{CF}_{3} \mathrm{COOH}$ can improve the quality of monolayers. This phenomenon has not yet been explored in this work. It is difficult to observe the evolution of monolayer formation as it is occurring. However, the results we have obtained with the use of the charge integrator to detect the surface stress in the piezoelectric cantilevers have enabled us to monitor SAM formation in real time.
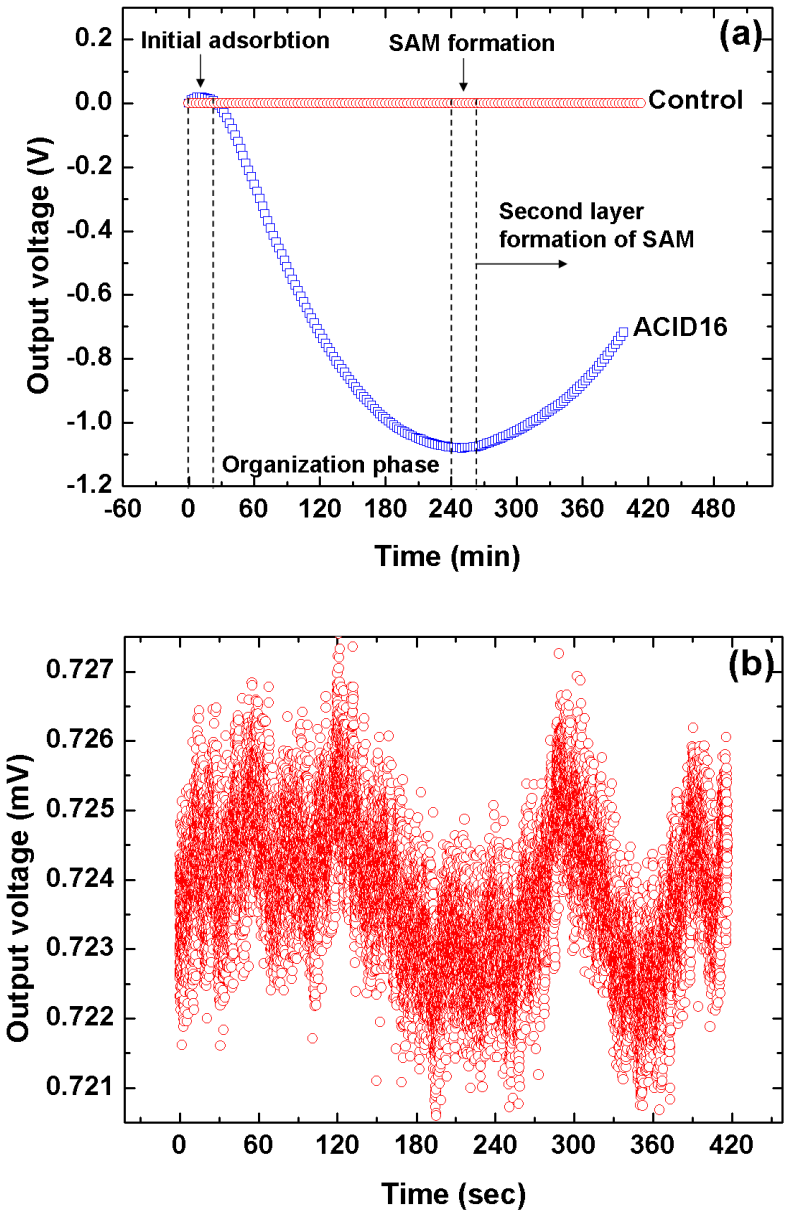

Figure 7.9. Reconstructed surface stress profile due to the formation of ACID16 on the Au-coated piezoelectric cantilever obtained from the sampled output voltage. (b) The magnification of the voltage response labeled control of a PVDF cantilever without an Au layer. 
In order to verify the presence of monolayers, a simple test to characterize the monolayer formation was performed using the microdroplet condensation technique [40, 41]. The Au-coated cantilever and the ACID16-SAM cantilever were cooled on a Peltier element below the dew point until droplets of ambient water condensed on the surface. The difference in size of water droplets for the Au surface and the ACID16-SAM surface appeared obviously as shown in figure 7.10. The droplets on the hydrophilic ACID16 monolayer are larger than those on the Au surface [17]. The size and density of droplets reflect different wettability properties of the cantilevers with and without the self-assembled monolayer, which indicate the presence of SAM.
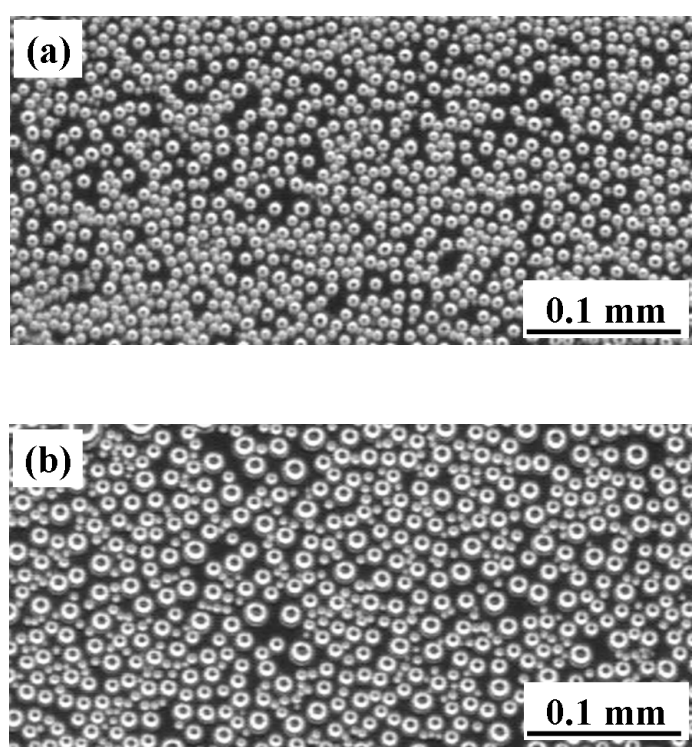

Figure 7.10. Optical microscope images of microdroplet condensation: (a) on Au surface, (b) on monolayer of ACID16.

\subsection{Conclusions}

A simple and efficient charge integration technique was proposed to measure the surface stress induced deflections in piezoelectric cantilevers. We demonstrated the use of the charge integrator to detect the alkanethiols in liquid environment. Utilizing this detection technique, the operation of piezoelectric cantilevers in static mode becomes possible. This charge integrator based detection system provides numerous advantages such as real-time, in-situ, low-cost detection. Furthermore, the deflection of piezoelectric cantilevers can be measured electrically without requiring external bulky optical devices, which enables the realization of portable sensor systems. Although, the charge integrator based detection system has been designed to operate with the peripheral electronics (computer, multimeter and function generator), it is capable of a stand-alone operation by using a microcontroller. The outcome of the study should incite to the establishments of the static detection 
method of piezoelectric cantilever sensors for chemical and biological applications. The charge integrator is not restricted to cantilevers: it can be applied to piezoelectric sensors in general. Currently, we are attempting to use our epitaxial piezoelectric MEMS devices with the charge integrator for sensing molecular interactions. The epitaxial piezoelectric MEMS on silicon demonstrate better performances compared to the polycrystalline piezoelectric devices [42]. This combination could improve significantly the detection sensitivity in the static way. 


\section{References}

[1] Muralt P 2000 PZT thin films for microsensors and actuators: Where do we stand? IEEE Trans. Ultrason. Ferroelectr. Freq. Control 47 903-15

[2] Muralt P 2000 Ferroelectric thin films for micro-sensors and actuators: a review $J$. Micromech. Microeng 10 136-46

[3] Damjanovic D, Muralt P and Setter N 2001 Ferroelectric sensors IEEE Sens. J. 1 191-206

[4] Trolier-McKinstry S and Muralt P 2004 Thin film piezoelectrics for MEMS J. Electroceram. 12 7-17

[5] Baborowski J 2004 Microfabrication of piezoelectric MEMS Integr. Ferroelectr. 66 3-17

[6] Muralt P 2008 Recent progress in materials issues for piezoelectric MEMS J. Am. Ceram. Soc. 91 1385-96

[7] Yi J W, Shih W Y, Mutharasan R and Shih W-H 2003 In situ cell detection using piezoelectric lead zirconate titanate-stainless steel cantilevers J. Appl. Phys. 93 619-625

[8] Campbell G A, Uknalis J, Tu S-I and Mutharasan R 2007 Detect of Escherichia coli O157:H7 in ground beef samples using piezoelectric excited millimeter-sized cantilever (PEMC) sensors Biosens. Bioelectron. 22 1296-1302

[9] Lee J H, Hwang K S, Park J, Yoon K H, Yoon D S and Kim T S 2005 Immunoassay of prostate-specific antigen (PSA) using resonant frequency shift of piezoelectric nanomechanical microcantilever Biosens. Bioelectron. 20 2157-2162

[10] Shin S, Kim J P, Sim S J and Lee J 2008 A multisized piezoelectric microcantilever biosensor array for the quantitative analysis of mass and surface stress Appl. Phys. Lett. 93102902

[11] Lu J, Ikehara T, Zhang Y, Mihara T, Itoh T and Maeda R 2007 High quality factor silicon cantilever transduced by piezoelectric lead zirconate titanate film for mass sensing applications Jpn. J. Appl. Phys. 46 7643-7647

[12] Kwon T Y, Eom K, Park J H, Yoon D S, Kim T S and Lee H L 2007 In situ real-time monitoring of biomolecular interactions based on resonating microcantilevers immersed in a viscous fluid Appl. Phys. Lett. 90223903

[13] Verbridge S S, Bellan L M, Parpia J M and Craighead H G 2006 Optically driven resonance of nanoscale flexural oscillators in liquid Nano Lett. 6 2109-2114

[14] Sone H, Ikeuchi A, Izumi T, Okano H and Hosaka S 2006 Femtogram mass biosensor using self-sensing cantilever for allergy check Jpn. J. Appl. Phys. 45 2301-2304

[15] Alvarez M, Calle A, Tamayo J, Lechuga L M, Abad A and Montoya A 2003 Development of nanomechanical biosensors for detection of the pesticide DDT Biosens. Bioelectron. 18 649653 
[16] Arntz Y, Seelig J D, Lang H P, Zhang J, Hunziker P, Ramseyer J P, Meyer E, Hegner M and Gerber Ch 2003 Label-free protein assay based on a nanomechanical cantilever array Nanotechnology 14 86-90

[17] Bietsch A, Zhang J, Hegner M, Lang H P and Gerber Ch 2004 Rapid functionalization of cantilever array sensors by inkjet printing Nanotechnology 15 873-880

[18] Helm M, Servant J J, Saurenbach F and Berger R 2005 Read-out of micromechanical cantilever sensors by phase shifting interferometry Appl. Phys. Lett. 87064101

[19] Plaza J A, Zinoviev K, Villanueva G, Alvarez M, Tamayo J, Dominquez C and Lechuga L M 2006 T-shaped microcantilever sensor with reduced deflection offset Appl. Phys. Lett. 89 094109

[20] Velanki S and Ji H -F 2006 Detection of feline coronavirus using microcantilever sensors Meas. Sci. Technol. 17 2964-2968

[21] Ji H -F, Hansen $\mathrm{K} \mathrm{M}, \mathrm{Hu} \mathrm{Z}$ and Thundat $\mathrm{T}$ Detection of $\mathrm{pH}$ variation using modified microcantilever sensors Sens. Actuators, B 72 233-238

[22] Park K T, Klafter R D and Bloomfield P E 1986 A charge readout algorithm for piezoelectric force transducers Sixth IEEE International Symposium on Applications of Ferroelectrics 715717

[23] Shepard Jr. J F, Moses P J and Trolier-McKinstry S 1998 The wafer flexure technique for the determination of the transverse piezoelectric coefficient $\left(d_{31}\right)$ of PZT thin films Sens. Actuators, A 71 133-138

[24] Shepard Jr. J F, Chu F, Kanno I and Trolier-McKinstry S 1999 Characterization and aging response of the $d_{31}$ piezoelectric coefficient of lead zirconate titanate thin films $J$. Appl. Phys. 85 6711-6716

[25] Xu F, Chu F and Trolier-McKinstry S 1999 Longitudinal piezoelectric coefficient measurement for bulk ceramics and thin films using pneumatic pressure rig J. Appl. Phys. 86 588-594

[26] Fukunaga M and Uesu Y 2003 Compact and simple apparatus for measuring direct piezoelectricity Jpn. J. Appl. Phys. 42 6115-6117

[27] Fu D, Ishikawa K, Minakata M and Suzuki H 2001 Observation of piezoelectric relaxation in ferroelectric thin films by continuous charge integration Jpn. J. Appl. Phys. 40 5683-5686

[28] Salvatori S, Masarone N, Di Nucci G, and Conte G 2006 Compact front-end electronics for low-level current sensor measurements Electron. Lett 42 682-684

[29] Shirinov A V and Schomburg W K 2008 Pressure sensor from a PVDF film Sens. Actuators, A 142 48-55

[30] "IVC102 Precision switched integrator transimpedance amplifier," datasheet, Burr-Brown Corporation. 
[31] Wiederkehr R S, Salvadori M C, Brugger J, Degasperi F T, and Cattani M 2007 Fabrication and testing of a poly(vinylidene fluoride) (PVDF) microvalve for gas flow control. Smart Mater. Struct. 16 2302-2307

[32] Mertens J, Calleja M, Ramos D, Taryn A, and Tamayo J 2007 Role of the gold film nanostructure on the nanomechanical response of microcantilever sensors J. Appl. Phys. 101 034904.1-034904.8

[33] Kohale S, Molina S M, Weeks B L, Khare R and Hope-Weeks L J 2007 Monitoring the formation of self-assembled monolayers of alkanedithiols using a micromechanical cantilever sensor Langmuir 23 1258-1263

[34] Nakamura F, Ito E, Hayashi T and Hara M 2006 Fabrication of COOH-terminated selfassembled monolayers for DNA sensors Colloids Surf., A 284-285 495-498

[35] Kumar A, Biebuyck H A and Whitesides G M 1994 Patterning self-assembled monolayers: Applications in materials science Langmuir 10 1498-1511

[36] Delamarche E, Schmid H, Bietsch A, Larsen N B, Rothuizen H, Michel B and Biebuyck H 1998 Transport mechanisms of alkanethiols during microcontact printing on gold $J$. Phys. Chem. B 102 3324-3334

[37] Ulman A 1996 Formation and structure of self-assembled monolayers Chem. Rev. 96 15331554

[38] Dai Z and Ju N 2001 Effect of chain length on the surface properties of $\omega$-carboxy alkanethiol self-assembled monolayers Phys. Chem. Chem. Phys. 3 3769-3773

[39] Wang H, Chen S, Li L and Jiang S 2005 Improved method for the preparation of carboxylic acid and amine terminated self-assembled monolayers of alkanethiolates Langmuir 212633 2636

[40] Hofer R, Textor M and Spencer N D 2001 Imaging of surface heterogeneity by the microdroplet condensation technique Langmuir 17 4123-4125

[41] Pardo L, Cris Wilson W and Boland T 2003 Characterization of patterned self-assembled monolayers and protein arrays generated by the ink-jet method Langmuir 19 1462-1466

[42] Isarakorn D, Sambri A, Janphuang P, Briand D, Gariglio S, Triscone J -M, Guy F, Reiner J W, Ahn C H and de Rooij N F 2010 Epitaxial piezoelectric MEMS on silicon J. Micromech. Microeng. 20055008 



\section{Chapter 8}

\section{Conclusions and outlook}

This thesis dealt with several aspects that are necessary for the realization of piezoelectric MEMS based on epitaxial PZT thin films. The PZT thin films epitaxially grown on silicon wafers through oxide layers exhibit high crystalline quality and excellent piezoelectric properties. The micropatterning techniques and process flow optimized for the epitaxial oxide layers deposited on silicon substrates have been developed. The success of integration of PZT thin films on silicon with appropriate microfabrication allows the superior properties of the epitaxial PZT thin films to be utilized in piezoelectric MEMS devices, i.e. large amplitude actuation with lower driving voltage, high sensitivity, and high efficiency in energy conversion. Two applications have been successfully demonstrated. First, different PZT/Si cantilever structures with and without a Si proof mass were realized for vibration energy harvesting applications. The optimized device has shown a high power density, while maintaining a low optimal resistive load. Second, the performance of a Si membrane actuated by an epitaxial PZT thin film was evaluated for localized-mass sensing applications, achieving a mass sensitivity in the order of $10^{-12} \mathrm{~g} \mathrm{~Hz}^{-1}$ with a minimum detectable mass of $5 \mathrm{ng}$. The charge integration technique was also investigated for measuring the deflection of piezoelectric cantilevers in static mode.

The PZT thin films investigated in this work were epitaxially grown on silicon substrates through the STO buffer layers and SRO oxide electrodes. $\mathrm{Pb}\left(\mathrm{Zr}_{0.2} \mathrm{Ti}_{0.8}\right) \mathrm{O}_{3}$ was chosen for its good lattice match with the STO and SRO layers. The epitaxial PZT thin films on silicon exhibit high crystalline quality, large remanent polarization, and excellent piezoelectric properties, which are important requirements for piezoelectric MEMS. The epitaxial PZT layers were grown onto 2" $\mathrm{Si}$ (001) wafers with a thickness up to $500 \mathrm{~nm}$. The residual stress was obtained within the range of 60$100 \mathrm{MPa}$ depending on the thickness of the epitaxial PZT films. Such a small level of residual stress could not deteriorate the behaviors of devices. The effective piezoelectric coefficient $e_{31, f}$ of $18.2 \pm$ $0.9 \mathrm{C} \mathrm{m}^{-2}$ was estimated for the $\mathrm{Pb}\left(\mathrm{Zr}_{0.2} \mathrm{Ti}_{0.8}\right) \mathrm{O}_{3}$ thin films without a poling treatment. This value is excellent when compared to the ones previously reported. However, the performance of piezoelectric MEMS devices is not only dependent on the piezoelectric coefficient, but also on the dielectric constant, since the figures of merit for various criteria depend on these two properties. Therefore, further investigation is required to understand the properties of epitaxial PZT thin films with different compositions. Moreover, for better reproducibility, improved sensitivity and higher throughput, the growth of a thicker epitaxial PZT layer on larger silicon wafers needs to be considered. 
The integration of epitaxial PZT layers on silicon substrates through the SRO and STO oxide layers requires specific microfabrication processes. These are the patterning methods for the metallic oxide SRO electrodes and the epitaxial PZT thin films. The ion milling process with alternating etching and cooling steps was successfully demonstrated to pattern the PZT/SRO/STO multilayer stack in a single processing step, allowing for the use of standard photoresist masks. The influence of the processing temperature and of the type of materials used as top electrodes on the epitaxial PZT film properties was also investigated. The results reveal that the thermal effect is a primary cause of the degradation of the properties of piezoelectric thin films covered with top electrode materials. Therefore, the electrode materials must be carefully selected, deposited and patterned at the end of the fabrication process to avoid the loss of ferroelectricity during the process. Epitaxial PZT cantilevers and membranes were fabricated using the optimized processes, exhibiting superior behaviors: high deflection at low driving voltage in static mode and strong harmonic oscillation with a high quality factor in dynamic mode. It is worth noting that in order to use such epitaxial PZT devices as actuators, further measurements of zero-force displacement would be required. As a conclusion, one can say that the microfabrication processes with associated process flow proposed in this thesis are optimized for the epitaxial PZT MEMS since no degradation in the piezoelectric properties was observed throughout the processes. However, the metal top electrodes should be replaced with epitaxial SRO top electrodes to improve fatigue characteristics for stability and reliability in final devices and their operation at higher temperature conditions. The use of DRIE should also be considered for structuring $\mathrm{PZT/SRO} / \mathrm{STO}$ heterostructures instead of ion milling, since the etch rate and selectivity could be improved. Alternatively, it has been demonstrated that it is possible to use wet etching for patterning the epitaxial PZT thin films followed by laser micromachining to structure the SRO/STO layers in some cases where lateral resolution and photoresist undercut of wet patterning are not issues.

The superior properties of the epitaxial PZT thin film and the effectiveness of the optimized microfabrication processes have been verified by two examples of epitaxial PZT MEMS devices. First, the performance of vibration energy harvesting devices based on the epitaxial PZT thin film has been evaluated. A high power density of up to $14 \mu \mathrm{W} g^{-2}$ was obtained, which is higher than the other piezoelectric energy harvesters surveyed in the literature. This is due to the high quality of the $c$-axis oriented PZT layer resulting in a high piezoelectric coefficient and a low dielectric constant (high figure of merit for power generation). The epitaxial PZT harvesters exhibit also high current generation with usable voltage, while maintaining lower optimal resistive load. Obviously, the epitaxial PZT thin film can improve overall electrical performances of piezoelectric energy harvesting devices. However, further improvements would require the use of the silicon-on-insulator substrates to obtain thinner and uniform silicon cantilever beam to reduce the resonant frequency and the development of the power management circuits in order to realize fully-functional vibration energy harvesting systems. The rule of thumb for their design is to increase the effective mass of the structure by adding a heavy proof mass which can provide low resonant frequency, high output power and 
compactness, simultaneously. The robustness of the harvesting devices should also be investigated for applications where high acceleration is required.

The second application focused on an epitaxial PZT membrane. The study of the basic dynamic characteristics of such structures has shown excellent results, i.e., a $150 \mathrm{~nm}$ thick epitaxial PZT film exhibits a strong harmonic oscillation response with a high quality factor at atmospheric pressure. The aim was to produce a resonant sensor for localized-mass sensing applications. As a localized-mass sensor, the mass sensitivity is a strong function of the mass position and the vibration mode, being highest when placing mass at the antinode and operating the sensor at higher mode of resonance. The epitaxial PZT membrane achieved the mass sensitivity of $46 \times 10^{-12} \mathrm{~g} \mathrm{~Hz}^{-1}$ for the 02 mode resonance with the minimum detectable mass of $5 \mathrm{ng}$ that demonstrates interesting opportunities in various biological and chemical sensing applications. Further enhancement of the mass sensing sensitivity can be achieved by reducing the size of a membrane. The finite element analysis predicts that the mass sensitivity will approach $10^{-15} \mathrm{~g} \mathrm{~Hz}^{-1}$, when the epitaxial PZT membrane is reduced to $500 \mu \mathrm{m}$ in diameter with the silicon thickness of $5 \mu \mathrm{m}$. In this domain, a further improvement would address the controlled deposition of functional layers, which is the key to convert the epitaxial PZT membrane into biological or chemical sensors. It is also worth to evaluate the mass sensing performance of this epitaxial PZT membrane with the distributed-mass loading in order to fully understand its behaviors and to be able to compare its performances with other mass sensors.

In the last part of this thesis, a measurement technique based on a charge integrator, which can electrically and statically measure the deflection of piezoelectric sensors, was developed. The proposed technique has been verified and demonstrated by means of detecting the alkanethiols in liquid environment using the Au-coated PVDF cantilevers. Experimental results revealed the potential of the charge integration technique to cope with the static measurement in piezoelectric sensors, which is a promising technique for the detection of biological molecules in a liquid environment. This measurement technique is not limited to only chemical and biological detection: it can be applied to a variety of piezoelectric sensors, such as impact sensors, piezo switches, fluid level sensors, and flow sensors. It is worth noting that in order to meet the desired application requirements, the influence of integration and reset periods on the accuracy of the measurements should be studied. Moreover, the charge integrator is not restricted to only PVDF piezoelectric materials; it can be used with various types of piezoelectric materials in general.

In summary, it has been demonstrated in this study that the properties of epitaxial PZT thin films lead to piezoelectric MEMS with superior performances. However, some issues have to be investigated to further improve the performances of piezoelectric MEMS based on epitaxial PZT thin films. In piezoelectric MEMS, the performances of the devices are governed by two major properties: the piezoelectric coefficient and the dielectric constant, which are strongly dependent on the $\mathrm{Zr} / \mathrm{Ti}$ compositions of the PZT thin films. Thus, the first issue that has to be addressed is the study of properties of epitaxial PZT thin films with different $\mathrm{Zr} / \mathrm{Ti}$ compositions to find the optimal 
composition for specific applications. Second, the optimal deposition process of epitaxial PZT thin films on large silicon wafers should be developed in order to achieve high fabrication throughput, which could be of high interest to manufacture epitaxial piezoelectric MEMS. Third, the thickness of the epitaxial PZT films should also be considered since it was reported that the piezoelectric coefficients were found to be higher for PZT films with higher thicknesses. Fourth, it is of interest to investigate the characteristics of multilayered dielectric/ferroelectric thin films and superlattices for MEMS devices. It has been reported that they can enhance the dielectric, ferroelectric, and pyroelectric properties with respect to the conventional ferroelectric thin films. Thus, it is expected to be an efficient way to better improve the piezoelectric properties and reduce the leakage currents.

The integration of epitaxial perovskite thin films on silicon is a promising technology, which could allow advanced MEMS to be realized. It can be applied to a wide range of devices, including high frequency resonators, RF switches, micromirrors, lab-on-a-chip systems, piezoelectric transformers, or high-sensitivity sensors. As progress is made towards nanoscale applications, where uniform control of the piezoelectric response is required at nanometer scale, the highly controllable piezoelectric properties of the epitaxial PZT thin films on silicon substrates can also offer several possibilities for the development of high performance nanoelectromechanical systems (NEMS). 


\section{Acknowledgements}

Although only one name appears on the spine of this thesis, it takes a team of talented people to run this project together. I have had the opportunity to work and collaborate with a wide range of people throughout this research. These collaborations have been the most important part of this thesis. Many people have contributed in different ways to this work. I would like to thank everyone who has been helpful and inspiring during my $\mathrm{PhD}$ studies.

First, I deeply thank Prof. Nico de Rooij for giving me the opportunity to work on an exciting project at the SAMLAB. My gratitude goes to my supervisor, Dr. Danick Briand, for full-heartedly support and guidance during my $\mathrm{PhD}$ studies. His interest in work, and his expertise and patience have sustained the project and me personally, for which I am very grateful. I warmly appreciate his genuine interest in me, my family, and his friendship.

Throughout this research, it has been a pleasure to work on this project with Prof. Jean-Marc Triscone, Dr. Stefano Gariglio, and Dr. Alessia Sambri, University of Geneva; Florian Guy, HEPIA; and Prof. Charles Ahn and Dr. James Reiner, Yale University. Their energetic work and their expertise in the epitaxial oxides undoubtedly lead to great advancement in this research field.

Prof. Herbert Shea and his team at LMTS have aided in the dynamic measurements with the laser Doppler vibrometer. Prof. Herbert Keppner and Henri Haquette, HE-ARC, have been a tremendous support on the laser micromachining. Their support I am grateful.

Special thanks go to all the students: Yexian Wu, Pattanaphong Janphuang, Sara Talei, and Michael Linder, who have contributed to this work through their master thesis and semester projects.

My interaction with the many lab mates has made my time at the SAMLAB a very enjoyable one. I would like to thank all the members of the EnviroMEMS group: Jerome Courbat, David de Koninck, Rahel Strassle, Rokaya Gueye, Pattanaphong Janphuang, Francisco Molina Lopez, Andres Vasquez Quintero, and Caglar Ataman for scientific assistance and activities we spent together. I appreciate the willing and capable help of Karine Frossard, who has helped with many admistrative works, and also Claudio Novelli for computer problem. At the risk of leaving someone out, I would also like to show my gratitude to all present and former colleagues of the SAMLAB and the LMTS for the warm friendship and care during the years: Muhamed Niklaus, Olivier Frey, Michael Zickar, Alexandra Homsy, Vincent Linder, Peter van der Wal, Wilfried Noell, Milena Koudelka-Hep Nicolas Golay, Luca Berdondini, Silvia Generelli, Philippe Dubois, Renato Krpoun, Anpan Han, Daniel Parrat, Kaspar Suter, Raphaël Imer, Yves Pétremand, Samuel Rosset, Severin Waldis, Dara Bayat, Roland Bitterli, Terunobu Akyiama, Sebastian Gautsch, Patrick Carazzetti, Friedjof Heuck, Fabio Jutzi, Sébastien Lani, Jonathan Masson, Luca Ribetto, Stefan Weber, Philip Wägli, and Frédéric Loizeau. 
I greatly appreciate the support for the microfabrication and SEM from the CSEM's staffs: Edith Millotte, Laurent Guillot, Sylvain Jeanneret, Sylviane Pochon, Eduardo Santoli, Pierre-André Clerc, Giovanno Bergonzi, Rémy Fournier, Stéphane Ischer, Sabina Jenny, José Vaquera, Massoud Dadras, and Mireille Leboeuf.

I acknowledge financial support by the Swiss National Science Foundation through the National Centre of Competence in Research 'Materials with Novel Electronic Properties' MaNEP. I am also grateful to the Ministry of Science and Technology, Thailand for the financial support for my $\mathrm{PhD}$ studies at EPFL IMT-NE SAMLAB.

I would like to thank my colleagues of the Reptechnics GmbH: Tao Sriphian-em, Bala Devan Balachandran, Lee Marcinkovski, Betsy Marcano, and Isabel Schmitter, for the warm friendship. I really had the great time working with all of you.

With all my heart, I am grateful to my parents for their unconditional and constant love, precious support, and confidence in me. When I feel discouraged, they always say "Trust in God He will see you through". My deepest gratitude goes to my wife Rachanok, for her patience, enthusiastic support, and understanding. For her support and partnership I will be always in debt. Thanks go to my wonderful little children, Isaiah and Elisha, who lift my spirits and serve as a continual reminder to me that my research work is not all that important.

Most importantly, my outmost thanks to God and his son Jesus Christ, for providing me the opportunity to step in the excellent world of science, for giving me the strength throughout this study, for always being there when I need Him, and for blessing me and my family with a wonderful life. Without God, this work will never be accomplished. May your name be exalted, honored, and glorified. 


\section{Publications}

\section{Peer-reviewed Journals}

D. Isarakorn, A. Sambri, P. Janphuang, D. Briand, S. Gariglio, J. -M. Triscone, F. Guy, J. W. Reiner, C. H. Ahn, and N. F. de Rooij, Epitaxial piezoelectric MEMS on silicon, Journal of Micromechanics and Microengineering, Vol. 20, 055008, 2010.

D. Isarakorn, M. Linder, D. Briand, and N. F. de Rooij, Evaluation of static measurement in piezoelectric cantilever sensors using a charge integration technique for chemical and biological detection, Measurement Science and Technology, Vol. 21, 075801, 2010.

D. Isarakorn, D. Briand, A. Sambri, S. Gariglio, J. -M. Triscone, F. Guy, J. W. Reiner, C. H. Ahn, and N. F. de Rooij, Finite element analysis and experiments on a silicon membrane actuated by an epitaxial PZT thin film for localized-mass sensing applications, Sensors and Actuators, B: Chemical, 2010, doi: 10.1016/j.snb.2010.10.009.

D. Isarakorn, D. Briand, P. Janphuang, A. Sambri, S. Gariglio, J. -M. Triscone, F. Guy, J. W. Reiner, C. H. Ahn, and N. F. de Rooij, The realization and performance of vibration energy harvesting MEMS devices based on an epitaxial piezoelectric thin film, Smart Materials and Structures (accepted).

D. Isarakorn, D. Briand, P. Janphuang, N. F. de Rooij, J. W. Reiner, and C. H. Ahn, Electrical characteristics of piezoelectric energy harvesters based on an epitaxial $\mathrm{Pb}\left(\mathrm{Zr}_{0.2} \mathrm{Ti}_{0.8}\right) \mathrm{O}_{3}$ thin film (submitted).

\section{Conference contributions}

D. Isarakorn, D. Briand, P. Janphuang, A. Sambri, S. Gariglio, J. -M. Triscone, F. Guy, J. W. Reiner, C. H. Ahn, and N. F. de Rooij, Energy harvesting MEMS device based on an epitaxial PZT thin film: fabrication and characterization, Proc. of the PowerMEMS 2010 Conference, pp. 203-206 Leuven, 2010. 
A. Sambri, S. Gariglio, J. -M. Triscone, D. Isarakorn, D. Briand, and N. F. de Rooij, Epitaxial thin films on silicon for MEMS devices, Presented at The $1^{\text {st }}$ National Congress on Functional Oxides for Electronics, Sorrento, 2009.

D. Isarakorn, D. Briand, S. Gariglio, A. Sambri, N. Stucki, J. -M. Triscone, F. Guy, J. W. Reiner, C. H. Ahn, and N. F. de Rooij, A study on epitaxial piezoelectric thin films grown on silicon for energy scavenging applications, Proc. of the PowerMEMS 2008 Conference, Vol.1, pp. 269-272, Sendai, 2008.

D. Isarakorn, M. Linder, D. Briand, and N. F. de Rooij, Detection of surface stress induced deflections in piezoelectric cantilever sensors using a charge integrator, Proc. of International Conference on Control, Automation and Systems, pp. 937-940, Seoul, 2008.

D. Isarakorn, D. Briand, S. Gariglio, A. Sambri, N. Stucki, J. -M. Triscone, F. Guy, S. -H. Baek, C. B. Eom, J. W. Reiner, C. H. Ahn, and N. F. de Rooij, Establishment of a technology platforms for epitaxial piezoelectric MEMS, Proc. of the Eurosensors XXII Conference, Vol.1, pp. 819-822, Dresden, 2008.

S. Gariglio, N. Stucki, J. -M. Triscone, D. Isarakorn, D. Briand, N. F. de Rooij, S. -H. Baek, C. -B. Eom, J. W. Reiner, and C. H. Ahn, MEMS fabrication based on epitaxial piezoelectric thin films on silicon, Presented at The $3^{\text {rd }}$ International Conference on Smart Materials, Structures and Systems, Sicily, 2008.

S. Gariglio, N. Stucki, J. -M. Triscone, D. Isarakorn, D. Briand, N. F. de Rooij, S. -H. Baek, C. -B. Eom, J. W. Reiner, and C. H. Ahn, MEMS fabrication based on epitaxial piezoelectric thin films on silicon, Presented at Workshop on Oxide Electronics 15, Colorado, 2008. 


\section{Biography}

Don Isarakorn was born in 1977 in Bangkok, Thailand. He received his B.Eng. degree in Electronics Engineering and M.Eng. degree in Control Engineering from King Mongkut's Institute of Technology Ladkrabang (KMITL), Thailand, in 2000 and 2003, respectively. In 1999, he spent a year at the National University of Electro-Communications, Japan, as an exchange student in the Japanese University Studies in Science \& Technology (JUSST) program. During staying in Japan, he worked in the field of micro robotics at the Applid Micro Systems Laboratory of Prof. Hisayuki Aoyama. This motivated him to perform his master thesis on the "Position control of ultrasonic linear motor using a pseudo-derivative control with feedforward gains (PDFF) controller designed by the Coefficient Diagram Method (CDM)" at the Control and Mechatronics Laboratory of Prof. Jongkol Ngamwiwit.

Apart from his thesis work, he was also involved in various research projects, for example, Speed/Position Control of Traveling-Wave Ultrasonic Motor, Optimal Control of Inverted Pendulum, Speed Control of Two-Mass System, Application of Shape-Memory Alloy (SMA) to Miniature Manipulator, and Design of Electrochemical-Metallizing Power Supply. In 2001, he was awarded the training program in the Technical Education Based on Micromachine Technology and Cultural Interchange between Young Engineers by the Asia/Pacific Culture Center for UNESCO, hosted by Department of Intelligent Mechanical Systems Engineering, Kagawa University, Japan. In 2003, he joined the Department of Control Engineering, KMITL as a lecturer, where his research activities focused on robotics, mechatronics, and control system design. Since 2006, he has been committee member of the International Micro-Mechanisms Contest organized by the Japan Society for Precision Engineering and the International Micro Robot Maze Contest held by Nagoya University and the city of Nagoya.

In 2004, he was awarded the scholarship by the Ministry of Science and Technology, Royal Thai Government to further his study towards the completion of Ph.D. degree in the field of Microengineering. He joined the Sensors, Actuators and Microsystems Laboratory (SAMLAB), headed by Prof. Nico de Rooij in April 2006. He worked since then with Dr. Danick Briand, the team leader of Energy and Environmental Microsystems (EnviroMEMS) group and with Prof. Jean-Marc Triscone, University of Geneva on the development of MEMS devices based on an epitaxial piezoelectric thin film on silicon. His research interests include piezoelectric materials for sensing and actuating applications, MEMS process development, and energy harvesting systems. 
